\title{
Z/m-GRADED LIE ALGEBRAS AND PERVERSE SHEAVES, II
}

\author{
GEORGE LUSZTIG AND ZHIWEI YUN
}

\begin{abstract}
We consider a fixed block for the equivariant perverse sheaves with nilpotent support on the 1-graded component of a semisimple cyclically graded Lie algebra. We give a combinatorial parametrization of the simple objects in that block.
\end{abstract}

\section{Contents}

\begin{tabular}{l|l|} 
Introduction & 322 \\
10. The vector space $\mathbf{V}$ and the sesquilinear form $(:)$ & 323 \\
11. The $\mathcal{A}$-lattice $\mathbf{V}_{\mathcal{A}}$ & 337 \\
12. Purity properties & 342 \\
13. An inner product & 343 \\
14. Odd vanishing & 348 \\
References & 353 \\
\hline
\end{tabular}

\section{INTRODUCTION}

As in $[\mathrm{LY}]$ we fix $G$, a semisimple simply connected algebraic group over $\mathbf{k}$ (an algebraically closed field of characteristic $p \geq 0$ ) and a $\mathbf{Z} / m$-grading $\mathfrak{g}=\oplus_{i \in \mathbf{Z} / m} \mathfrak{g}_{i}$ for the Lie algebra $\mathfrak{g}$ of $G$; here $m$ is an integer $>0$ and $p$ is 0 or a large prime number. We fix $\eta \in \mathbf{Z}-\{0\}$ and let $\delta$ be the image of $\eta$ in $\mathbf{Z} / m$. Let $G_{\underline{0}}$ be the closed connected subgroup of $G$ with Lie algebra $\mathfrak{g}_{0}$; this group acts naturally on $\mathfrak{g}_{\delta}$ and on $\mathfrak{g}_{\delta}^{\text {nil }}=\mathfrak{g}_{\delta} \cap \mathfrak{g}^{\text {nil }}$ where $\mathfrak{g}^{\text {nil }}$ is the variety of nilpotent elements in $\mathfrak{g}$. Recall that $\mathcal{I}\left(\mathfrak{g}_{\delta}\right)$ denotes the set of pairs $(\mathcal{O}, \mathcal{L})$ where $\mathcal{O}$ is a $G_{\underline{0}}$-orbit on $\mathfrak{g}_{\delta}^{\text {nil }}$ and $\mathcal{L}$ is an irreducible $G_{\underline{0}}$-equivariant local system on $\mathcal{O}$ up to isomorphism. Let $\mathfrak{B}$ be the set of isomorphism classes of simple $G_{\underline{0}}$-equivariant perverse sheaves on $\mathfrak{g}_{\delta}^{\text {nil }}$. Then there is a natural bijection $\mathcal{I}\left(\mathfrak{g}_{\delta}\right) \rightarrow \mathfrak{B}$ given by $(\mathcal{O}, \mathcal{L}) \mapsto \mathcal{L}^{\sharp}[\operatorname{dim} \mathcal{O}]$ (for the notation $\mathcal{L}^{\sharp}$ see [LY, 0.11]). In [LY, Theorem 0.6] we have shown that $\mathfrak{B}$ can be naturally decomposed as a disjoint union of blocks ${ }^{\xi} \mathfrak{B}$ indexed by the $G_{\underline{0}^{-}}$ conjugacy classes of admissible systems $\xi=\left(M, M_{0}, \mathfrak{m}, \mathfrak{m}_{*}, \tilde{C}\right)$ such that if $B, \bar{B}^{\prime}$ are in different blocks then the $G_{\underline{0}}$-equivariant Ext-groups of $B$ with $B^{\prime}$ are zero. This generalizes a result of $[\mathrm{L} 4$ in the $\mathbf{Z}$-graded case; its analogue in the ungraded case is the known partition of the set $G$-equivariant simple perverse sheaves on $\mathfrak{g}^{\text {nil }}$ into blocks given by the generalized Springer correspondence.

In this paper we give an (essentially) combinatorial way to parametrize the objects in a fixed block $\xi_{\mathfrak{B}}$ of $\mathfrak{B}$. It is known that in the ungraded case, the objects in

Received by the editors October 12, 2016, and, in revised form, June 23, 2017.

2010 Mathematics Subject Classification. Primary 20 G99.

The first author was supported by NSF grant DMS-1566618.

The second author was supported by NSF grant DMS-1302071 and the Packard Foundation. 
a fixed block are indexed by the irreducible representations of a certain (relative) Weyl group attached to the block. In the $\mathbf{Z} / m$-graded case we will associate to the block $\xi_{\mathfrak{B}}$ a $\mathbf{Q}$-vector space $\mathbf{E}$ with a certain finite collection of hyperplanes. The complement of the union of these hyperplanes is naturally a union of finitely many (not necessarily conical) chambers which can be taken to form a basis of a $\mathbf{Q}(v)$-vector space $\mathbf{V}^{\prime}$ (here $v$ is an indeterminate). The chambers represent various spiral induction associated to the block. The vector space $\mathbf{V}^{\prime}$ carries a natural, explicit, sesquilinear form (:) : $\mathbf{V}^{\prime} \times \mathbf{V}^{\prime} \rightarrow \mathbf{Q}(v)$ defined in terms of dimensions of Ext-groups between the spiral inductions (see [LY, 6.4]) which correspond to the chambers. We show that the left radical of this form is the same as its right radical. Taking the quotient of $\mathbf{V}^{\prime}$ by the left or right radical we obtain a $\mathbf{Q}(v)$-vector space $\mathbf{V}$ with an induced sesquilinear form (:). It turns out that $\mathbf{V}$ is naturally isomorphic to the Grothendieck group based on $\xi_{\mathfrak{B}}$ (tensored with $\mathbf{Q}(v)$ ) and, in particular, the number of elements in $\xi_{\mathfrak{B}}$ is equal to $\operatorname{dim}_{\mathbf{Q}(v)} \mathbf{V}$. The vector space $\mathbf{V}$ has a natural bar involution ${ }^{-}: \mathbf{V} \rightarrow \mathbf{V}$ and a natural $\mathcal{A}$-lattice $\mathbf{V}_{\mathcal{A}}$ in $\mathbf{V}$, both defined combinatorially. (Here $\mathcal{A}=\mathbf{Z}\left[v, v^{-1}\right]$.) We then define a subset $\mathbf{B}^{\prime}$ of $\mathbf{V}$ as the set of all $b \in \mathbf{V}_{\mathcal{A}}$ such that $\bar{b}=b$ and $(b: b) \in 1+v \mathbf{Z}[[v]]$. We show that $\mathbf{B}^{\prime}$ is a signed basis of $\mathbf{V}$. (Although the definition of $\mathbf{B}^{\prime}$ is combinatorial, the proof of the fact that it is a well-defined signed basis is based on geometry; it is not combinatorial. It would be desirable to find a proof without using geometry.) Let $\mathbf{B}^{\prime} / \pm$ be the set of orbits of the $\mathbf{Z} / 2$-action $b \mapsto-b$ on $\mathbf{B}^{\prime}$. We show that $\mathbf{B}^{\prime} / \pm$ is in natural bijection with the given block $\xi \mathfrak{B}$. Thus $\mathbf{B}^{\prime} / \pm$ could be regarded as a combinatorial index set for ${ }^{\xi} \mathfrak{B}$. (A similar result in the $\mathbf{Z}$-graded case appears in [L4].)

We now discuss the contents of the various sections. In Section 10 we define the $\mathbf{Q}$-vector vector $\mathbf{E}$ with its hyperplane arrangement associated to a block. We also define the $\mathbf{Q}(v)$-vector space $\mathbf{V}^{\prime}$ with its sesquilinear form (:), its quotient space $\mathbf{V}$ and the bar operator. In Section 11 we define the $\mathcal{A}$-lattice $\mathbf{V}_{\mathcal{A}}$ in $\mathbf{V}$ and the signed basis $\mathbf{B}^{\prime}$. In Section 12 we prove some purity properties of the cohomology sheaves of the simple $G_{\underline{0}}$-equivariant perverse sheaves on $\mathfrak{g}_{\delta}^{\text {nil }}$, which generalize those in the $\mathbf{Z}$-graded case given in [L4]. In Section 13 we generalize an argument in [L4] to express the matrix whose entries are the values of the (:)-pairing at two elements of $\mathfrak{B}$ as a product of three matrices. This is used in Section 14 to prove the vanishing of the odd cohomology sheaves of the intersection cohomology of the closure of any $G_{\underline{0}}$-orbit in $\mathfrak{g}_{\delta}^{\text {nil }}$ with coefficients in an irreducible $G_{\underline{0}^{-}}$equivariant local system on that orbit. This generalizes a result in $[\mathbf{L} 4$ in the $\mathbf{Z}$-graded case whose proof was quite different (it was based on geometric arguments which are not available in the present case).

We will adhere to the notation and assumptions of [LY].

\section{The Vector space V And the SESquilinear form (:)}

In this section, we introduce a combinatorial way of calculating the number of irreducible perverse sheaves in a block ${ }^{\xi} \mathcal{Q}\left(\mathfrak{g}_{\delta}\right)$. This is achieved by introducing a finite-dimensional vector space $\mathbf{V}^{\prime}$ over $\mathbf{Q}(v)$ together with a sesquilinear form on it coming from the pairing between spiral inductions in the fixed block $\xi$. Then the number of irreducible perverse sheaves in ${ }^{\xi} \mathcal{Q}\left(\mathfrak{g}_{\delta}\right)$ turns out to be the rank of this sesquilinear form on $\mathbf{V}^{\prime}$ (Proposition 10.19). 
10.1. We fix $\xi \in \underline{\mathfrak{I}}_{\eta}$ and a representative $\dot{\xi}=\left(M, M_{0}, \mathfrak{m}, \mathfrak{m}_{*}, \tilde{C}\right) \in \mathfrak{T}_{\eta}$ for $\xi$. We also fix $\phi=(e, h, f) \in J^{M}$ such that $e \in \stackrel{\circ}{\mathfrak{m}}_{\eta}, h \in \mathfrak{m}_{0}, f \in \mathfrak{m}_{-\eta}$. We set $\iota=\iota_{\phi} \in Y_{M}$. Let

$$
Z=\mathcal{Z}_{M}^{0}
$$

Since $Z$ is a torus, $Y_{Z}$ is naturally a free abelian group (with operation written as addition) and $\mathbf{E}:=Y_{Z, \mathbf{Q}}$ may be naturally identified with the $\mathbf{Q}$-vector space $\mathbf{Q} \otimes Y_{Z}$. Let $X_{Z}=\operatorname{Hom}\left(Y_{Z}, \mathbf{Z}\right)$ and let $\langle:\rangle$ be the obvious perfect bilinear pairing $Y_{Z} \times X_{Z} \rightarrow \mathbf{Z}$. This extends to a bilinear pairing $\mathbf{E} \times\left(\mathbf{Q} \otimes X_{Z}\right) \rightarrow \mathbf{Q}$ denoted again by $\langle:\rangle$. For any $\alpha \in X_{Z}$ let

$$
\mathfrak{g}^{\alpha}=\{x \in \mathfrak{g} ; \operatorname{Ad}(z) x=\alpha(z) x \quad \forall z \in Z\} .
$$

For any $(\alpha, i) \in X_{Z} \times \mathbf{Z} / m$ let

$$
\mathfrak{g}_{i}^{\alpha}=\mathfrak{g}^{\alpha} \cap \mathfrak{g}_{i} .
$$

For any $(\alpha, n, i) \in X_{Z} \times \mathbf{Z} \times \mathbf{Z} / m$ let

$$
\mathfrak{g}_{i}^{\alpha, n}=\mathfrak{g}_{i}^{\alpha} \cap\left({ }_{n}^{\iota} \mathfrak{g}_{i}\right) .
$$

For any $i \in \mathbf{Z} / m$, let $\mathcal{R}_{i}=\left\{(\alpha, n) \in X_{Z} \times \mathbf{Z} ; \mathfrak{g}_{i}^{\alpha, n} \neq 0\right\}, \mathcal{R}_{i}^{*}=\left\{(\alpha, n) \in \mathcal{R}_{i} ; \alpha \neq 0\right\}$. Note that

(a) $\operatorname{dim} \mathfrak{g}_{i}^{\alpha, n}=\operatorname{dim} \mathfrak{g}_{-i}^{-\alpha,-n}$ for any $\alpha, n, i$; hence $(\alpha, n) \mapsto(-\alpha,-n)$ is a bijection $\mathcal{R}_{i} \stackrel{\sim}{\longrightarrow} \mathcal{R}_{-i}$ and a bijection $\mathcal{R}_{i}^{*} \stackrel{\sim}{\longrightarrow} \mathcal{R}_{-i}^{*}$.

We have

$$
\mathfrak{g}=\oplus_{(\alpha, n, i) \in X_{Z} \times \mathbf{Z} \times \mathbf{Z} / m}\left(\mathfrak{g}_{i}^{\alpha, n}\right)=\oplus_{i \in \mathbf{Z} / m,(\alpha, n) \in \mathcal{R}_{i}}\left(\mathfrak{g}_{i}^{\alpha, n}\right) .
$$

For any $N \in \mathbf{Z}$ and any $(\alpha, n) \in \mathcal{R}_{\underline{N}}^{*}$ we set

$$
\mathfrak{H}_{\alpha, n, N}=\{\varpi \in \mathbf{E} ;\langle\varpi: \alpha\rangle=2 N / \eta-n\} .
$$

This is an affine hyperplane in $\mathbf{E}$. We set

$$
\mathbf{E}^{\prime}=\mathbf{E}-\cup_{N \in \mathbf{Z},(\alpha, n) \in \mathcal{R}_{\underline{\underline{N}}}^{*}} \mathfrak{H}_{\alpha, n, N} .
$$

10.2. Recall the notation $Y_{H, \mathbf{Q}}$ for a connected algebraic group $H$ with Lie algebra $\mathfrak{h}$ from [LY] 0.11]. For $\mu, \mu^{\prime} \in Y_{H, \mathbf{Q}}$, we say $\mu$ commutes with $\mu^{\prime}$ if for some $r, r^{\prime}$ in $\mathbf{Z}_{>0}$ such that $r \mu, r^{\prime} \mu^{\prime} \in Y_{H}$, the images of the homomorphisms $r \mu, r^{\prime} \mu^{\prime}: \mathbf{k}^{*} \rightarrow H$ commute with each other. This property is independent of the choice of $r, r^{\prime}$. If $\mu$ commutes with $\mu^{\prime}$, and $r, r^{\prime} \in \mathbf{Z}_{>0}$ are as above, then letting $\lambda=r \mu, \lambda^{\prime}=r^{\prime} \mu^{\prime}$, we have the homomorphism $\nu: \mathbf{k}^{*} \rightarrow H$ given by $\nu(t)=\lambda\left(t^{r^{\prime}}\right) \lambda^{\prime}\left(t^{r}\right)$. We then define $\mu+\mu^{\prime}:=\nu /\left(r r^{\prime}\right) \in Y_{H, \mathbf{Q}}$. Then $\mu+\mu^{\prime}$ is independent of the choice of $r, r^{\prime}$. Moreover, for any $\kappa \in \mathbf{Q}$, we have

$$
{ }_{\kappa}^{\mu+\mu^{\prime}} \mathfrak{h}=\oplus_{s, t \in \mathbf{Q} ; s+t=\kappa}\left({ }_{s}^{\mu} \mathfrak{h} \cap{ }_{t}^{\mu^{\prime}} \mathfrak{h}\right) .
$$

Let $\varpi \in \mathbf{E}$. Since $\varpi \in \mathbf{E}=Y_{Z, \mathbf{Q}}$ commutes with $\iota \in Y_{M_{0}}$, by the above discussion, we may define

$$
\underline{\varpi}=\frac{|\eta|}{2}(\varpi+\iota) \in Y_{M_{0}, \mathbf{Q}} \subset Y_{G_{\underline{0}}, \mathbf{Q}} .
$$

From the definitions, $\varpi$ may be computed as follows. We choose $f \in \mathbf{Z}_{>0}$ such that $\lambda^{\prime}:=f \varpi \in Y_{Z}$ and $f /|\eta| \in \mathbf{Z}$; we define $\lambda \in Y_{G_{0}}$ by $\lambda(t)=\iota\left(t^{f}\right) \lambda^{\prime}(t)=\lambda^{\prime}(t) \iota\left(t^{f}\right)$ for all $t \in \mathbf{k}^{*}$; we then have $\underline{\varpi}=\frac{|\eta|}{2 f} \lambda \in Y_{G_{\underline{0}}}, \mathbf{Q}$.

We shall set $\epsilon=\dot{\eta} \in\{1,-1\}$ (the sign of $\eta$, see 0.12 ).

Now ${ }^{\epsilon} \mathfrak{p}_{*}^{\varpi}, \epsilon \tilde{i}^{\frac{\varpi}{*}}$ are well-defined; see [LY, 2.5, 2.6]. 
We set

$$
\epsilon \tilde{\mathfrak{l}} \underline{\varpi}=\oplus_{N \in \mathbf{Z}}^{\epsilon \tilde{\mathfrak{l}} \frac{\varpi}{N}} .
$$

We show:

(b) We have ${ }^{\epsilon \tilde{\mathfrak{l}}_{*}}=\mathfrak{m}_{*}$ if and only if $\varpi \in \mathbf{E}^{\prime}$.

If $x \in \mathfrak{g}_{i}^{\alpha, n}$ and $t \in \mathbf{k}^{*}$, then

$$
\operatorname{Ad}(\lambda(t)) x=\operatorname{Ad}\left(\iota\left(t^{f}\right)\right) \operatorname{Ad}\left(\lambda^{\prime}(t)\right) x=t^{n f+\left\langle\lambda^{\prime}: \alpha\right\rangle} x
$$

and $\operatorname{Ad}(\iota(t)) x=t^{n} x$. Thus, $\mathfrak{g}_{i}^{\alpha, n} \subset{ }_{n f+\left\langle\lambda^{\prime}: \alpha\right\rangle}^{\lambda} \mathfrak{g}_{i}$ and $\mathfrak{g}_{i}^{\alpha, n} \subset{ }_{n}^{\iota} \mathfrak{g}_{i}$. It follows that for any $k \in \mathbf{Z}$ we have

$$
\begin{gathered}
{ }_{k}^{\lambda} \mathfrak{g}_{i}=\oplus_{(\alpha, n) \in \mathcal{R}_{i} ; n f+\left\langle\lambda^{\prime}: \alpha\right\rangle=k}\left(\mathfrak{g}_{i}^{\alpha, n}\right), \\
{ }_{k} \mathfrak{g}_{i}=\oplus_{(\alpha, n) \in \mathcal{R}_{i} ; n=k}\left(\mathfrak{g}_{i}^{\alpha, n}\right) .
\end{gathered}
$$

For any $N \in \mathbf{Z}$ we have $\epsilon \tilde{\mathfrak{l}} \frac{\varpi}{N}={ }_{2 f N / \eta}^{\lambda} \underline{\mathfrak{g}_{N}}$. We see that

$$
\epsilon \tilde{\mathfrak{l}} \frac{\varpi}{N}=\oplus_{(\alpha, n) \in \mathcal{R}_{\underline{N}} ; n f+\left\langle\lambda^{\prime}: \alpha\right\rangle=2 f N / \eta}\left(\mathfrak{g}_{\underline{N}}^{\alpha, n}\right) .
$$

Recall from $1.2(\mathrm{e})$ that if $N \in \mathbf{Z}, \mathfrak{m}_{N} \neq 0$, then $N \in \eta \mathbf{Z}$. Let $N \in \eta \mathbf{Z}$. From the arguments in 3.3 (or by [LY, 10.3(a)]) we see that, for some $\varpi^{\prime} \in \mathbf{E}$, we have $\tilde{\epsilon} \tilde{\mathfrak{l}}_{*}^{\prime}{ }^{\prime}=\mathfrak{m}_{*}$. (Here $\underline{\varpi}^{\prime}$ is attached to $\varpi^{\prime}$ in the same way that $\underline{\varpi}$ was attached to $\varpi$.) Hence, using (e) with $\varpi$ replaced by $\varpi^{\prime}$, we see that there exists a subset $R_{N}$ of $\mathcal{R}_{\underline{N}}$ such that $\mathfrak{m}_{N}=\oplus_{(\alpha, n) \in R_{N}}\left(\mathfrak{g}_{N}^{\alpha, n}\right)$. If $(\alpha, n) \in R_{N}$, then $0 \neq \mathfrak{g}_{N}^{\alpha, n} \subset \mathfrak{m}_{N}$. Hence for $x \in \mathfrak{g}_{\underline{N}}^{\alpha, n}-\{0\}$ and $z \in Z, t \in \mathbf{k}^{*}$, we have $\operatorname{Ad}(z) x=\alpha(z) x, \overline{\operatorname{Ad}} d(\iota(t)) x=t^{n} x$; but $\operatorname{Ad}(z) \bar{x}=x$ since $Z$ is in the center of $M$ and $\operatorname{Ad}(\iota(t)) x=t^{2 N / \eta} x$ since $x \in \mathfrak{m}_{N}$. Thus $\alpha(z)=1$ (so that $\alpha=0$ ) and $n=2 N / \eta$. We see that $R_{N} \subset\{(0,2 N / \eta)\}$. Conversely, we show that $\mathfrak{g}_{\underline{N}}^{0,2 N / \eta} \subset \mathfrak{m}_{N}$. If $x \in \mathfrak{g}_{\underline{N}}^{0,2 N / \eta}$, then $\operatorname{Ad}(z) x=x$ for all $z \in Z$ and $\operatorname{Ad}(\iota(t)) x=t^{2 N / \eta} x$ for all $t \in \mathbf{k}^{*}$. Since $\dot{\xi}$ in 9.1 is admissible, there exists $t_{0} \in \mathbf{k}^{*}, z \in Z$, such that $\mathfrak{m}$ is the fixed point set of $\operatorname{Ad}\left(\iota\left(t_{0}\right) z\right) \theta: \mathfrak{g} \rightarrow \mathfrak{g}$. Since $\mathfrak{m}_{\eta}$ is contained in this fixed point set and $\operatorname{Ad}(z)$ acts trivially on it, we see that $t_{0}^{2} \zeta^{\eta} y=y$ for all $y \in \mathfrak{m}_{\eta}$.

If $\mathfrak{m}_{\eta} \neq 0$, it follows that $t_{0}^{2} \zeta^{\eta}=1$. Thus we have $\operatorname{Ad}(\iota(t) z) \theta(x)=t_{0}^{2 N / \eta} \zeta^{N} x=$ $\left(t_{0}^{2} \zeta^{\eta}\right)^{N / \eta} x=x$. (Here we use that $N \in \eta \mathbf{Z}$.) Thus $x$ is contained in the fixed point set of $\operatorname{Ad}\left(\iota\left(t_{0}\right) z\right) \theta: \mathfrak{g} \rightarrow \mathfrak{g}$, so that $x \in \mathfrak{m}_{N}$. We see that for any $N \in \eta \mathbf{Z}$ we have

$$
\mathfrak{m}_{N}=\mathfrak{g}_{\underline{N}}^{0,2 N / \eta} .
$$

Now (f) also holds when $\mathfrak{m}_{\eta}=0$. (In that case we must have $\iota=1$ hence $\mathfrak{m}=\mathfrak{m}_{0}$, so that $\mathfrak{m}_{N}=0$ for $N \neq 0$. Moreover, by [LY, 3.6(d)], $\mathfrak{m}$ is a Cartan subalgebra of $\mathfrak{g}_{\underline{0}}$ and $Z=M$. We also have $\mathfrak{g}_{\underline{N}}^{0,2 N / \eta}=0$ for $N \neq 0$. Thus, for $N \neq 0$, (f) states that $0=0$. If $N=0$, (f) states that $\mathfrak{m}$ is its own centralizer in $\mathfrak{g}_{\underline{0}}$, which is clear.)

From (e),(f) we see that $\mathfrak{m}_{N} \subset \epsilon \tilde{\mathfrak{l}} \frac{\mathrm{w}}{N}$ for all $N \in \eta \mathbf{Z}$ and that

(g) for $N \in \eta \mathbf{Z}$ we have $\mathfrak{m}_{N}=\tilde{\epsilon}^{\epsilon} \frac{\varpi}{N}$ if and only if the following holds: $\{(\alpha, n) \in$ $\left.\mathcal{R}_{\underline{N}} ; n+\langle\varpi: \alpha\rangle=2 N / \eta\right\}$ is equal to $\{(0,2 N / \eta)\}$ if $(0,2 N / \eta) \in \mathcal{R}_{\underline{N}}$ and is empty if $(0,2 N / \eta) \notin \mathcal{R}_{\underline{N}}$;

$\left(\mathrm{g}^{\prime}\right)$ for $N \in \overline{\mathbf{Z}}-\eta \mathbf{Z}$ we have $\mathfrak{m}_{N}={ }^{\epsilon} \mathfrak{i}_{\frac{\varpi}{N}}$ (or equivalently $\epsilon \tilde{\mathfrak{l}} \frac{\varpi}{N}=0$ ) if and only if $\left\{(\alpha, n) \in \mathcal{R}_{\underline{N}} ; n+\langle\varpi: \alpha\rangle=2 N / \eta\right\}=\emptyset$. The condition in (g) can be also expressed 
as follows:

$\left\{(\alpha, n) \in \mathcal{R}_{N}^{*} ; n+\langle\varpi: \alpha\rangle=2 N / \eta\right\}=\emptyset$ for any $N \in \eta \mathbf{Z}$.

The condition in $\left(\mathrm{g}^{\prime}\right)$ can be also expressed as follows:

$\left\{(\alpha, n) \in \mathcal{R}_{N} ; n+\langle\varpi: \alpha\rangle=2 N / \eta\right\}=\emptyset$ for any $N \in \mathbf{Z}-\eta \mathbf{Z}$.

Indeed, it is enough to show that if $N \in \mathbf{Z}-\eta \mathbf{Z}$ and $\left\{(\alpha, n) \in \mathcal{R}_{N} ; n+\langle\varpi: \alpha\rangle=\right.$ $2 N / \eta\}$, then we have automatically $\alpha \neq 0$. (Assume that $\alpha=0$. Then $n=2 N / \eta$ so that $n$ is an odd integer. Since $\mathfrak{g}^{0}=\mathfrak{m}$ and $\mathfrak{g}_{N}^{0, n} \neq 0$ we see that ${ }_{n}^{\iota} \mathfrak{m} \neq 0$. Using $1.2(\mathrm{~d})$ we deduce that $n$ is even, a contradiction.)

We see that (b) holds.

From (c) we deduce that

$$
\epsilon_{\mathfrak{p}} \frac{\varpi}{N}=\oplus_{k \in \mathbf{Z} ; k \geq 2 f N / \eta}\left({ }_{k}^{\lambda} \mathfrak{g}_{\underline{N}}\right)=\oplus_{k \in \mathbf{Z},(\alpha, n) \in \mathcal{R}_{\underline{N}} ; k \geq 2 f N / \eta, n f+\left\langle\lambda^{\prime}: \alpha\right\rangle=k}\left(\mathfrak{g}_{\underline{N}}^{\alpha, n}\right),
$$

hence

$$
\epsilon \mathfrak{p}^{\frac{\varpi}{N}}=\oplus_{(\alpha, n) \in \mathcal{R}_{\underline{N}}} ;\langle\varpi: \alpha\rangle \geq 2 N / \eta-n\left(\mathfrak{g}_{\underline{N}}^{\alpha, n}\right) .
$$

The nilradical $\epsilon_{\mathfrak{u}_{*}}$ of $\epsilon_{\mathfrak{p}} \frac{\varpi}{*}$ is given by

$$
\epsilon \mathfrak{u} \frac{\varpi}{N}=\oplus_{\left.(\alpha, n) \in \mathcal{R}_{\underline{N}}^{*} ; \varpi: \alpha\right\rangle>2 N / \eta-n}\left(\mathfrak{g}_{\underline{N}}^{\alpha, n}\right) .
$$

We see that for $\varpi, \varpi^{\prime}$ in $\mathbf{E}$ and $N \in \mathbf{Z}$, the following two conditions are equivalent:

(I) ${ }^{\epsilon} \mathfrak{p}_{N}^{\varpi}={ }^{\epsilon} \mathfrak{p}_{N}{ }^{\prime}$.

(II) For any $(\alpha, n) \in \mathcal{R}_{\underline{N}}^{*}$ we have $\langle\varpi: \alpha\rangle+n \geq 2 N / \eta \Leftrightarrow\left\langle\varpi^{\prime}: \alpha\right\rangle+n \geq 2 N / \eta$.

For $\varpi, \varpi^{\prime}$ in $\mathbf{E}^{\prime}$ we say that $\varpi \equiv \varpi^{\prime}$ if for any $N \in \mathbf{Z}$ and any $(\alpha, n) \in \mathcal{R}_{\underline{N}}^{*}$ we have

$$
(\langle\varpi: \alpha\rangle+n-2 N / \eta)\left(\left\langle\varpi^{\prime}: \alpha\right\rangle+n-2 N / \eta\right)>0 .
$$

This is clearly an equivalence relation on $\mathbf{E}^{\prime}$. From the equivalence of (I),(II) above, we see that

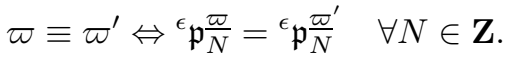

For any $\varpi \in \mathbf{E}^{\prime}$ we set

$$
\begin{aligned}
& I_{\varpi}={ }^{\epsilon} \operatorname{Ind}_{\epsilon_{\mathfrak{p}^{\varpi}}}^{\mathfrak{g}_{\delta}}\left(\tilde{C}\left[-\operatorname{dim} \mathfrak{m}_{\eta}\right]\right) \in \mathcal{Q}\left(\mathfrak{g}_{\delta}\right), \\
& \tilde{I}_{\varpi}=\widetilde{\operatorname{Ind}}_{\epsilon_{\mathfrak{p}} \frac{\varpi}{\eta}}^{\mathfrak{g}_{\delta}}(\tilde{C}) \in \mathcal{Q}\left(\mathfrak{g}_{\delta}\right) \text {. }
\end{aligned}
$$

Here we regard $\tilde{C}$ as an object of $\mathcal{Q}\left(\tilde{\mathfrak{l}}_{\eta}^{\varpi}\right)=\mathcal{Q}\left(\mathfrak{m}_{\eta}\right)$, see (b). Note that in $\mathcal{K}\left(\mathfrak{g}_{\delta}\right)$ we have

$$
\tilde{I}_{\varpi}=v^{h(\varpi)} I_{\varpi}
$$

where

$$
h(\varpi)=\operatorname{dim} \epsilon_{\mathfrak{u}} \frac{\varpi}{0}+\operatorname{dim} \epsilon_{\mathfrak{u}} \frac{\varpi}{\eta}+\operatorname{dim} \mathfrak{m}_{\eta}=\operatorname{dim} \epsilon_{\mathfrak{u}} \frac{\varpi}{0}+\operatorname{dim} \epsilon_{\mathfrak{p}} \frac{\varpi}{\eta} .
$$

We show:

(l) If $\varpi, \varpi^{\prime} \in \mathbf{E}^{\prime}, \varpi \equiv \varpi^{\prime}$, then $I_{\varpi}=I_{\varpi^{\prime}}, h(\varpi)=h\left(\varpi^{\prime}\right)$.

Indeed, in this case we have ${ }^{\epsilon} \mathfrak{p}^{\frac{\varpi}{N}}={ }^{\epsilon} \mathfrak{p}^{\frac{\varpi^{\prime}}{N}}$ for all $N \in \mathbf{Z}$ (see (j)) and the result follows from the definitions. 
10.3. We keep the setup of 10.1, 10.2. As in [LY, 2.9], for any $N \in \mathbf{Z}$ we set

$$
\tilde{\mathfrak{l}}_{N}^{\phi}={ }_{2 N / \eta}^{\iota} \mathfrak{g}_{\underline{N}} \text { if } 2 N / \eta \in \mathbf{Z}, \quad \tilde{\mathfrak{l}}_{N}^{\phi}=0 \text { if } 2 N / \eta \notin \mathbf{Z} .
$$

Hence

$$
\tilde{\mathfrak{l}}_{N}^{\phi}=\oplus_{(\alpha, n) \in \mathcal{R}_{\underline{N}} ; n=2 N / \eta} \mathfrak{g}_{\underline{N}}^{\alpha, n} .
$$

We set $\tilde{\mathfrak{l}}^{\phi}=\oplus_{N \in \mathbf{Z}} \tilde{\mathfrak{l}}_{N}^{\phi}$.

Let

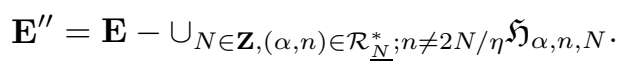

For $\varpi \in \mathbf{E}$ we show:

(b) We have ${ }^{\epsilon \tilde{\mathfrak{l}} \underline{\varpi}} \subset \tilde{\mathfrak{l}}^{\phi}$ if and only if $\varpi \in \mathbf{E}^{\prime \prime}$.

Using (a) and 10.2(e) we see that we have $\oplus_{N} \epsilon \tilde{\mathfrak{l}}_{N} \overline{\underline{w}} \subset \tilde{\mathfrak{l}}^{\phi}$ if and only if for any $N \in \mathbf{Z}$ we have

$$
\left\{(\alpha, n) \in \mathcal{R}_{\underline{N}} ; n+\langle\varpi: \alpha\rangle=2 N / \eta\right\} \subset\left\{(\alpha, n) \in \mathcal{R}_{\underline{N}} ; n=2 N / \eta\right\},
$$

or equivalently,

$$
\left\{(\alpha, n) \in \mathcal{R}_{\underline{N}} ; n+\langle\varpi: \alpha\rangle=2 N / \eta ; n \neq 2 N / \eta\right\}=\emptyset,
$$

or equivalently,

$$
\left\{(\alpha, n) \in \mathcal{R}_{\underline{N}}^{*} ;\langle\varpi: \alpha\rangle=2 N / \eta-n \neq 0\right\}=\emptyset .
$$

This is the same as the condition that $\varpi \in \mathbf{E}^{\prime \prime}$. This proves (b).

10.4. We show:

(a) Let $\mathfrak{p}_{*}$ be an $\epsilon$-spiral with a splitting $\mathfrak{m}_{*}^{\prime}$ such that $\mathfrak{m}$ is a Levi subalgebra of a parabolic subalgebra of $\mathfrak{m}^{\prime}=\oplus_{N} \mathfrak{m}_{N}^{\prime}$ compatible with the $\mathbf{Z}$-gradings. Then for some $\varpi \in \mathbf{E}$ we have $\mathfrak{p}_{*}={ }^{\epsilon} \mathfrak{p}_{*}^{\varpi}, \mathfrak{m}_{*}^{\prime}={ }^{\epsilon} \tilde{\mathfrak{l}}_{*}^{\varpi}$.

We can find $\mu \in Y_{G_{0}}, \mathbf{Q}$ such that $\mathfrak{p}_{*}={ }^{\epsilon} \mathfrak{p}_{*}^{\mu}$ and $\mathfrak{m}_{*}^{\prime}=\epsilon_{\mathfrak{l}_{*}^{\mu}}^{\mu}$. Let $M_{0}^{\prime}=e^{\mathfrak{m}_{0}^{\prime}}$. We choose $f \in \mathbf{Z}_{>0}$ such that $\lambda_{1}:=f \mu \in Y_{G_{\underline{0}}}$. We have $\mathfrak{m}_{N}^{\prime}={ }_{f N \epsilon}^{\lambda_{1}} \mathfrak{g}_{N}$ for all $N \in \mathbf{Z}$. Let $N \in \eta \mathbf{Z}$. Since $\mathfrak{m}_{N} \subset \mathfrak{m}_{N}^{\prime}$, for any $x \in \mathfrak{m}_{N}$ and any $t \in \mathbf{k}^{*}$ we have $\operatorname{Ad}\left(\lambda_{1}(t)\right) x=t^{f N \epsilon} x$; we have also $\operatorname{Ad}(\iota(t)) x=t^{2 N / \eta} x$.

Hence $\operatorname{Ad}\left(\lambda_{1}\left(t^{2}\right) \iota\left(t^{-f|\eta|}\right)\right) x=x$ for any $x \in \mathfrak{m}_{N}$. Since this holds for any $N \in \eta \mathbf{Z}$, it follows that the image of $\lambda_{1}^{2} \iota^{-f|\eta|}: \mathbf{k}^{*} \rightarrow M_{0}^{\prime}$ commutes with $M$. Since $M$ is a Levi subgroup of a parabolic subgroup of $M^{\prime}$, the image of $\lambda_{1}^{2} \iota^{-f|\eta|}$ is contained in $\mathcal{Z}_{M}$, hence in $\mathcal{Z}_{M}^{0}=Z$. In particular, the images of $\iota$ and $\lambda_{1}^{2} \iota^{-f|\eta|}$ commute with each other, hence the images of $\lambda_{1}$ and $\iota$ commute with each other. It therefore makes sense to write $\lambda^{\prime}:=2 \lambda_{1}-f|\eta| \iota \in Y_{M}$ and we actually have $\lambda^{\prime} \in Y_{Z}$. Let $\varpi=|\eta|^{-1} f^{-1} \lambda^{\prime} \in Y_{Z, \mathbf{Q}}=\mathbf{E}$. We have $\varpi=|\eta|^{-1}(2 \mu-|\eta| \iota)$ hence $\varpi+\iota=2|\eta|^{-1} \mu$, that is, $\mu=\underline{\varpi}$ and (a) is proved.

Let $\mathcal{C}^{\prime}$ be the collection of $\epsilon$-spirals $\mathfrak{p}_{*}$ such that $\mathfrak{m}_{*}$ is a splitting of $\mathfrak{p}_{*}$. We show:

(b) $\mathcal{C}^{\prime}$ coincides with the collection of $\epsilon$-spirals of the form ${ }^{\epsilon} \mathfrak{p}_{*}^{\varpi}$ with $\varpi \in \mathbf{E}^{\prime}$.

Assume first that $\mathfrak{p}_{*} \in \mathcal{C}^{\prime}$. Using (a) with $\mathfrak{m}_{*}^{\prime}=\mathfrak{m}_{*}$ we see that for some $\varpi \in \mathbf{E}$ we have $\mathfrak{p}_{*}={ }^{\epsilon} \mathfrak{p}_{*}^{\varpi}, \mathfrak{m}_{*}={ }^{\epsilon} \tilde{\mathfrak{l}}_{*}^{\varpi}$. Using $10.2(\mathrm{~b})$, we see that $\varpi \in \mathbf{E}^{\prime}$.

Conversely, assume that $\mathfrak{p}_{*}={ }^{\epsilon} \mathfrak{p}_{*}^{\varpi}$ for some $\varpi \in \mathbf{E}^{\prime}$. From 10.2(b) we have $\mathfrak{m}_{*}=\tilde{\mathfrak{l}}_{\mathfrak{i}}$. . Thus $\mathfrak{p}_{*} \in \mathcal{C}^{\prime}$. This proves (b). 
Let $\mathcal{C}^{\prime \prime}$ be the collection of $\epsilon$-spirals $\mathfrak{p}_{*}$ with the following property: there exists a splitting $\mathfrak{m}_{*}^{\prime}$ of $\mathfrak{p}_{*}$ such that $\mathfrak{m}_{N} \subset \mathfrak{m}_{N}^{\prime} \subset \tilde{\mathfrak{l}}_{N}^{\phi}$ for all $N$. We show:

(c) $\mathcal{C}^{\prime \prime}$ coincides with the collection of $\epsilon$-spirals of the form ${ }^{\epsilon} \mathfrak{p}_{*}^{\varpi}$ with $\varpi \in \mathbf{E}^{\prime \prime}$.

Assume first that $\mathfrak{p}_{*} \in \mathcal{C}^{\prime \prime}$ and let $\mathfrak{m}_{*}^{\prime}$ be a splitting of $\mathfrak{p}_{*}$ as in the definition of $\mathcal{C}^{\prime \prime}$. Now $\mathfrak{m}$ is a Levi subalgebra of a parabolic subalgebra of $\mathfrak{m}^{\prime}=\oplus_{N} \mathfrak{m}_{N}^{\prime}$ compatible with the $\mathbf{Z}$-gradings of $\mathfrak{m}$ and $\mathfrak{m}^{\prime}$. (Indeed, from the proof of $3.7(\mathrm{c})$ we see that there exists $\lambda \in Y_{Z}$ such that $\mathfrak{m}=\left\{y \in \tilde{\mathfrak{l}}^{\phi} ; \operatorname{Ad}(\lambda(t)) y=y \quad \forall t \in \mathbf{k}^{*}\right\}$. Since $\mathfrak{m}^{\prime} \subset \tilde{\mathfrak{l}}^{\phi}$ we see that $\mathfrak{m}=\left\{y \in \mathfrak{m}^{\prime} ; \operatorname{Ad}(\lambda(t)) y=y \quad \forall t \in \mathbf{k}^{*}\right\}$, as required.) Using (a), we see that for some $\varpi \in \mathbf{E}$ we have $\mathfrak{p}_{*}={ }^{\epsilon} \mathfrak{p}_{*}^{\varpi}, \mathfrak{m}_{*}^{\prime}={ }^{\epsilon} \tilde{\mathfrak{l}}_{*}^{\varpi}$. Since $\tilde{\mathfrak{l}}^{\varpi} \subset \tilde{\mathfrak{l}}_{N}^{\phi}$ for all $N$, we see from $10.3(\mathrm{~b})$ that $\varpi \in \mathbf{E}^{\prime \prime}$.

Conversely, assume that $\mathfrak{p}_{*}={ }^{\epsilon} \mathfrak{p}_{*}^{\varpi}$ for some $\varpi \in \mathbf{E}^{\prime \prime}$. Let $m_{*}^{\prime}={ }^{\epsilon} \tilde{\mathfrak{l}}_{*} \frac{\varpi}{*}$. From 10.3(b) we see that $\epsilon \tilde{\mathfrak{l}} \frac{\varpi}{N} \subset \tilde{\mathfrak{l}}_{N}^{\phi}$ for all $N$. We also have $\mathfrak{m}_{N} \subset \tilde{\mathfrak{l}}^{\tilde{\mathfrak{l}}} \frac{\varpi}{N}$ for all $N$. Thus $\mathfrak{p}_{*} \in \mathcal{C}^{\prime \prime}$. This proves (c).

Note that:

(d) If $\mathfrak{p}_{*} \in \mathcal{C}^{\prime \prime}$, then the splitting $\mathfrak{m}_{*}^{\prime}$ in the definition of $\mathcal{C}^{\prime \prime}$ is in fact unique.

Indeed, assume that $\mathfrak{m}_{*}^{\prime}, \tilde{\mathfrak{m}}_{*}^{\prime}$ are splittings of $\mathfrak{p}_{*}$ such that $\mathfrak{m}_{N} \subset \mathfrak{m}_{N}^{\prime}, \mathfrak{m}_{N} \subset \tilde{\mathfrak{m}}_{N}^{\prime}$ for all $N$. By [LY, 2.7] we can find $u \in U_{0}\left(U_{0}\right.$ as in [LY, 2.5]) such that $\operatorname{Ad}(u) \mathfrak{m}_{*}^{\prime}=$ $\tilde{\mathfrak{m}}_{*}^{\prime}$. In particular, we have $\operatorname{Ad}(u) \mathfrak{m}_{0}^{\prime}=\tilde{\mathfrak{m}}_{0}^{\prime}$. This implies $u=1$ since $\mathfrak{m}_{0}^{\prime}, \tilde{\mathfrak{m}}_{0}^{\prime}$ are both Levi subalgebras of $\mathfrak{p}_{0}$ containing $\mathfrak{m}_{0}$. Hence $\mathfrak{m}_{*}^{\prime}=\tilde{\mathfrak{m}}_{*}^{\prime}$.

10.5. Let $\mathfrak{p}_{*}, \mathfrak{p}_{*}^{\prime}$ be two $\epsilon$-spirals such that $\mathfrak{p}_{N} \subset \mathfrak{p}_{N}^{\prime}$ for all $N$. Let $\mathfrak{u}_{*}, \mathfrak{u}_{*}^{\prime}$ be their nilradicals. Then we have $\mathfrak{u}_{N}^{\prime} \subset \mathfrak{u}_{N}$ for all $N$. In particular, $\mathfrak{u}_{N}^{\prime} \subset \mathfrak{p}_{N}$ for all $N$. We show:

(a) $\oplus_{N} \mathfrak{p}_{N} / \mathfrak{u}_{N}^{\prime}$ is a parabolic subalgebra of $\mathfrak{l}^{\prime}=\oplus_{N} \mathfrak{p}_{N}^{\prime} / \mathfrak{u}_{N}^{\prime}$.

Let $P_{0}=e^{\mathfrak{p}_{0}}, P_{0}^{\prime}=e^{\mathfrak{p}_{0}^{\prime}}$ and let $U_{0}=U_{P_{0}}, U_{0}^{\prime}=U_{P_{0}^{\prime}}$. We have $P_{0} \subset P_{0}^{\prime}, U_{0}^{\prime} \subset U_{0}$. Now $\mathfrak{p}_{*}={ }^{\epsilon} \mathfrak{p}_{*}^{\mu}$ and $\mathfrak{p}_{*}^{\prime}={ }^{\epsilon} \mathfrak{p}_{*}^{\mu^{\prime}}$ for some $\mu=\lambda / r, \mu^{\prime}=\lambda^{\prime} / r^{\prime}$, with $\lambda, \lambda^{\prime} \in Y_{G_{0}}$ and $r, r^{\prime} \in \mathbf{Z}_{>0}$. We have $\lambda\left(\mathbf{k}^{*}\right) \subset P_{0}, \lambda^{\prime}\left(\mathbf{k}^{*}\right) \subset P_{0}^{\prime}$. We can find Levi subgroups $\tilde{L}_{0}$, $\tilde{L}_{0}^{\prime}$ of $P_{0}, P_{0}^{\prime}$ such that $\tilde{L}_{0} \subset \tilde{L}_{0}^{\prime}$. By conjugating $\lambda$ (resp. $\lambda^{\prime}$ ) by an element of $U_{0}$ (resp. $U_{0}^{\prime}$ ) we can assume that $\lambda \in \mathcal{Z}_{\tilde{L}_{0}}, \lambda^{\prime} \in \mathcal{Z}_{\tilde{L}_{0}^{\prime}}$. Since $\mathcal{Z}_{\tilde{L}_{0}^{\prime}} \subset \mathcal{Z}_{\tilde{L}_{0}}$, we have $\lambda(t) \lambda^{\prime}\left(t^{\prime}\right)=\lambda^{\prime}\left(t^{\prime}\right) \lambda(t)$ for any $t, t^{\prime}$ in $\mathbf{k}^{*}$. Hence we have $\mathfrak{g}=\oplus_{k, k^{\prime} \in \mathbf{Q}, i \in \mathbf{Z} / m}\left(\begin{array}{l}\mu, \mu^{\prime} \\ k, k^{\prime}\end{array} \mathfrak{g}_{i}\right)$, where ${ }_{k, k^{\prime}}^{\mu, \mu^{\prime}} \mathfrak{g}_{i}={ }_{k}^{\mu} \mathfrak{g}_{i} \cap{ }_{k^{\prime}}^{\mu^{\prime}} \mathfrak{g}_{i}$. We have

$$
\begin{aligned}
& \mathfrak{p}_{N}=\oplus_{k, k^{\prime} \in \mathbf{Q} ; k \geq N \epsilon}\left(\begin{array}{c}
\mu, \mu^{\prime} \\
k, k^{\prime} \\
\mathfrak{g}_{N}
\end{array}\right), \\
& \mathfrak{p}_{N}^{\prime}=\oplus_{k, k^{\prime} \in \mathbf{Q} ; k^{\prime} \geq N \epsilon}\left(\begin{array}{c}
\mu, \mu^{\prime} \\
k, k^{\prime}
\end{array} \mathfrak{g}_{\underline{N}}\right), \\
& \mathfrak{u}_{N}^{\prime}=\oplus_{k, k^{\prime} \in \mathbf{Q} ; k^{\prime}>N \epsilon}\left(\begin{array}{l}
\mu, \mu^{\prime} \\
k, k^{\prime}
\end{array} \mathfrak{g}_{\underline{N}}\right) .
\end{aligned}
$$

Since $\mathfrak{u}_{N}^{\prime} \subset \mathfrak{p}_{N} \subset \mathfrak{p}_{N}^{\prime}$ we see that

$$
\begin{aligned}
& \mathfrak{p}_{N}=\oplus_{k, k^{\prime} \in \mathbf{Q} ; k \geq N \epsilon, k^{\prime} \geq N \epsilon}\left(\begin{array}{c}
\mu, \mu^{\prime} \\
k, k^{\prime}
\end{array} \mathfrak{g}_{\underline{N}}\right), \\
& \mathfrak{u}_{N}^{\prime}=\oplus_{k, k^{\prime} \in \mathbf{Q} ; k \geq N \epsilon, k^{\prime}>N \epsilon}\left(\begin{array}{l}
\mu, \mu^{\prime} \\
k, k^{\prime}
\end{array} \mathfrak{g}_{\underline{N}}\right),
\end{aligned}
$$

hence

$$
\mathfrak{p}_{N} / \mathfrak{u}_{N}^{\prime} \cong \oplus_{k, k^{\prime} \in \mathbf{Q} ; k \geq N \epsilon, k^{\prime}=N \epsilon}\left(\begin{array}{l}
\mu, \mu^{\prime} \\
k, k^{\prime}
\end{array} \mathfrak{g}_{\underline{N}}\right) .
$$

This is a subspace of

$$
\mathfrak{p}_{N}^{\prime} / \mathfrak{u}_{N}^{\prime} \cong \tilde{\mathfrak{l}}_{N}^{\prime}:=\oplus_{k, k^{\prime} \in \mathbf{Q} ; k^{\prime}=N \epsilon}\left(\begin{array}{c}
\mu, \mu^{\prime} \\
k, k^{\prime} \\
\mathfrak{g}_{N}
\end{array}\right) .
$$


Since $\mu$ and $\mu^{\prime}$ commute with each other, it makes sense to define $\nu=\mu-$ $\mu^{\prime} \in Y_{\tilde{L}_{0}^{\prime}, \mathbf{Q}}$. Let $\tilde{\mathfrak{l}}^{\prime}=\oplus_{N} \tilde{\mathfrak{l}}_{N}^{\prime} \subset \mathfrak{g}$. Then $\tilde{L}_{0}^{\prime}$ acts on $\mathfrak{l}^{\prime}$ by the Ad-action and $\nu$ induces a $\mathbf{Q}$-grading $\mathfrak{l}^{\prime}=\oplus_{k_{1} \in \mathbf{Q}}{\underline{k_{1}}}^{\nu} \mathfrak{l}^{\prime}$. From the definitions we see that $\oplus_{N} \mathfrak{p}_{N}^{\prime} / \mathfrak{u}_{N}=$ $\oplus_{k_{1} \in \mathbf{Q} ; k_{1} \geq 0}\left(\begin{array}{c}\nu \\ k_{1}\end{array} \mathfrak{l}^{\prime}\right)$. Thus (a) holds.

A similar argument shows:

(b) If $\mathfrak{m}_{*}$ is a splitting of $\mathfrak{p}_{*}$, the obvious map $\mathfrak{m}=\oplus_{N} \mathfrak{m}_{N} \rightarrow \oplus_{N} \mathfrak{p}_{N} / \mathfrak{u}_{N}^{\prime}$ defines an isomorphism of $\mathfrak{m}$ onto a Levi subalgebra of $\oplus_{N} \mathfrak{p}_{N} / \mathfrak{u}_{N}^{\prime}$.

10.6. Let $\varpi, \varpi^{\prime}$ in $\mathbf{E}^{\prime}$. For any $t \in \mathbf{Q}$ such that $0 \leq t \leq 1$ we set

$$
\varpi_{t}:=t \varpi+(1-t) \varpi^{\prime}
$$

We assume that there is a unique hyperplane $\mathfrak{H}$ of the form $\mathfrak{H}=\mathfrak{H}_{\alpha_{0}, n_{0}, N_{0}}$ for some $\left(\alpha_{0}, n_{0}, N_{0}\right)$ with $N_{0} \in \mathbf{Z},\left(\alpha_{0}, n_{0}\right) \in \mathcal{R}_{\underline{N}_{0}}^{*}$ such that $\varpi_{t} \in \mathfrak{H}$ for some $t=s$; this $s$ is necessarily unique since $\varpi_{0} \notin \mathfrak{H}$. Note, however, that the triple $\left(\alpha_{0}, n_{0}, N_{0}\right)$ is not uniquely determined by $\mathfrak{H}$. We set $\varpi^{\prime \prime}=\varpi_{s}$,

$$
\begin{gathered}
\mathfrak{p}_{*}={ }^{\epsilon} \mathfrak{p}_{*}^{\varpi}, \mathfrak{p}_{*}^{\prime}={ }^{\epsilon} \mathfrak{p}_{*}^{\varpi^{\prime}}, \mathfrak{u}_{*}={ }^{\epsilon} \mathfrak{u}_{*}^{\varpi}, \mathfrak{u}_{*}^{\prime}={ }^{\epsilon} \mathfrak{u}_{*}^{\varpi^{\prime}}, \\
\mathfrak{p}_{*}^{\prime \prime}={ }^{\epsilon} \mathfrak{p}_{*}^{\varpi^{\prime \prime}}, \tilde{\mathfrak{l}}_{*}^{\prime \prime}={ }^{\epsilon} \tilde{\mathfrak{l}}_{*}{ }^{\prime \prime}, \tilde{\mathfrak{l}}^{\prime \prime}=\oplus_{N \in \mathbf{Z}} \tilde{\mathfrak{l}}_{N}^{\prime \prime}, \\
P_{0}=e^{\mathfrak{p}_{0}}, P_{0}^{\prime}=e^{\mathfrak{p}_{0}^{\prime}}, P_{0}^{\prime \prime}=e^{\mathfrak{p}_{0}^{\prime \prime}}
\end{gathered}
$$

We show:

(a) For any $N \in \mathbf{Z}$ we have $\mathfrak{p}_{N} \subset \mathfrak{p}_{N}^{\prime \prime}$; hence $P_{0} \subset P_{0}^{\prime \prime}$. For any $N \in \mathbf{Z}$ we have $\mathfrak{p}_{N}^{\prime} \subset \mathfrak{p}_{N}^{\prime \prime}$; hence $P_{0}^{\prime} \subset P_{0}^{\prime \prime}$.

Using $10.2(\mathrm{~h})$, we see that to prove the first sentence in (a) it is enough to show that for any $(\alpha, n) \in \mathcal{R}_{N}^{*}$ such that $\langle\varpi: \alpha\rangle \geq 2 N / \eta-n$ we have also $\left\langle\varpi^{\prime \prime}: \alpha\right\rangle \geq 2 N / \eta-n$. (Since $\varpi \in \mathbf{E}^{\prime}$, we must have $\langle\varpi: \alpha\rangle>2 N / \eta-n$. Since for $t \in \mathbf{Q}, s<t \leq 1$ we have $\varpi_{t} \in \mathbf{E}^{\prime}$, it follows that for all such $t$ we have $\left\langle\varpi_{t}: \alpha\right\rangle>2 N / \eta-n$. Taking the limit as $t \mapsto s$ we obtain $\left\langle\varpi^{\prime \prime}: \alpha\right\rangle \geq 2 N / \eta-n$, as required). The second sentence in (a) is proved in a similar way.

We show:

(b) Fix $N \in \mathbf{Z}$. If $\mathfrak{H} \neq \mathfrak{H}_{\alpha, n, N}$ for any $(\alpha, n) \in \mathcal{R}_{\underline{N}}^{*}$, then we have $\mathfrak{p}_{N}=\mathfrak{p}_{N}^{\prime \prime}=\mathfrak{p}_{N}^{\prime}$.

We first show that $\mathfrak{p}_{N}=\mathfrak{p}_{N}^{\prime \prime}$. By the equivalence of (I),(II) in 10.2, it is enough to show that for any $(\alpha, n) \in \mathcal{R}_{\underline{N}}^{*}$ we have

$$
\langle\varpi: \alpha\rangle+n-2 N / \eta>0 \Leftrightarrow\left\langle\varpi^{\prime \prime}: \alpha\right\rangle+n-2 N / \eta>0,
$$

or equivalently, that $c_{1} c_{s}>0$, where $c_{t}=\left\langle\varpi_{t}: \alpha\right\rangle+n-2 N / \eta$ for $t \in \mathbf{Q}$ such that $0 \leq t \leq 1$. From our assumptions we see that $c_{t} \neq 0$ for all $t$. It follows that either $c_{t}>0$ for all $t$ or $c_{t}<0$ for all $t$. In particular, $c_{1} c_{s}>0$, as required. The proof of the equality $\mathfrak{p}_{N}^{\prime}=\mathfrak{p}_{N}^{\prime \prime}$ is entirely similar.

We show:

(c) If $\mathfrak{H} \neq \mathfrak{H}_{\alpha, n, 0}$ for any $(\alpha, n) \in \mathcal{R}_{\underline{0}}^{*}$ and $\mathfrak{H} \neq \mathfrak{H}_{\alpha, n, \eta}$ for any $(\alpha, n) \in \mathcal{R}_{\delta}^{*}$, then $I_{\varpi}=I_{\varpi^{\prime}}, h(\varpi)=h\left(\varpi^{\prime}\right)$.

Indeed, in this case, by (b) we have $\mathfrak{p}_{N}=\mathfrak{p}_{N}^{\prime}$ for $N \in\{0, \eta\}$ and the equality $I_{\varpi}=I_{\varpi^{\prime}}$ follows from the definitions. From $\mathfrak{p}_{0}=\mathfrak{p}_{0}^{\prime}$ we deduce that $\mathfrak{u}_{0}=\mathfrak{u}_{0}^{\prime}$. Using this and $\mathfrak{p}_{\eta}=\mathfrak{p}_{\eta}^{\prime}$ we deduce that $h(\varpi)=h\left(\varpi^{\prime}\right)$. This proves (c).

We show:

(d) If $\mathfrak{H}=\mathfrak{H}_{\alpha_{0}, n_{0}, 0}$ for some $\left(\alpha_{0}, n_{0}\right) \in \mathcal{R}_{\underline{0}}^{*}$ but $\mathfrak{H} \neq \mathfrak{H}_{\alpha, n, \eta}$ for any $(\alpha, n) \in \mathcal{R}_{\delta}^{*}$, then $I_{\varpi} \cong I_{\varpi^{\prime}}, h(\varpi)=h\left(\varpi^{\prime}\right)$. 
By (a) we have $\mathfrak{p}_{N} \subset \mathfrak{p}_{N}^{\prime \prime}, \mathfrak{p}_{N}^{\prime} \subset \mathfrak{p}_{N}^{\prime \prime}$ for all $N \in \mathbf{Z}$ and by (b) we have $\mathfrak{p}_{\eta}=\mathfrak{p}_{\eta}^{\prime \prime}=\mathfrak{p}_{\eta}^{\prime}$. We can now apply [LY, 4.5(b)] twice to conclude that

$$
I_{\varpi} \cong \oplus_{j \in J} I_{\varpi^{\prime \prime}}\left[-2 a_{j}\right], I_{\varpi^{\prime}} \cong \oplus_{j^{\prime} \in J^{\prime}} I_{\varpi^{\prime \prime}}\left[-2 a_{j^{\prime}}^{\prime}\right]
$$

where $a_{j}$ (resp. $\left.a_{j^{\prime}}^{\prime}\right)$ are integers such that

$$
\rho_{P_{0}^{\prime \prime} / P_{0}} ! \overline{\mathbf{Q}}_{l}=\oplus_{j \in J} \overline{\mathbf{Q}}_{l}\left[-2 a_{j}\right], \rho_{P_{0}^{\prime \prime} / P_{0}^{\prime \prime} !} \overline{\mathbf{Q}}_{l}=\oplus_{j^{\prime} \in J^{\prime}} \overline{\mathbf{Q}}_{l}\left[-2 a_{j^{\prime}}^{\prime}\right] .
$$

To show that $I_{\varpi} \cong I_{\varpi \prime}$ it is then enough to show that

$$
\oplus_{j \in J} \overline{\mathbf{Q}}_{l}\left[-2 a_{j}\right] \cong \oplus_{j^{\prime} \in J^{\prime}} \overline{\mathbf{Q}}_{l}\left[-2 a_{j^{\prime}}^{\prime}\right]
$$

or that $\rho_{P_{0}^{\prime \prime} / P_{0}}$ ! $\overline{\mathbf{Q}}_{l} \cong \rho_{P_{0}^{\prime \prime} / P_{0}^{\prime} !} \overline{\mathbf{Q}}_{l}$. This is clear since $P_{0}^{\prime \prime} / P_{0}, P_{0}^{\prime \prime} / P_{0}^{\prime}$ are partial flag manifolds of the reductive quotient $L_{0}^{\prime \prime}$ of $P_{0}^{\prime \prime}$ with respect to two associate parabolic subgroups. The above argument shows also that $\operatorname{dim} U_{P_{0}}=\operatorname{dim} U_{P_{0}^{\prime}}$ hence $\operatorname{dim} \mathfrak{u}_{0}=$ $\operatorname{dim} \mathfrak{u}_{0}^{\prime}$. Moreover, from (b) we have $\mathfrak{p}_{\eta}=\mathfrak{p}_{\eta}^{\prime}$; we see that $h(\varpi)=h\left(\varpi^{\prime}\right)$. This proves (d).

For $N \in \mathbf{Z}$ let $\mathfrak{q}_{N}$ (resp. $\mathfrak{q}_{N}^{\prime}$ ) be the image of $\mathfrak{p}_{N}$ (resp. $\mathfrak{p}_{N}^{\prime}$ ) under the obvious projection $\mathfrak{p}_{N}^{\prime \prime} \rightarrow \tilde{\mathfrak{l}}_{N}^{\prime \prime}$. From $10.5(\mathrm{a}),(\mathrm{b})$, we see that $\mathfrak{q}=\oplus_{N} \mathfrak{q}_{N}, \mathfrak{q}^{\prime}=\oplus_{N} \mathfrak{q}_{N}^{\prime}$ are parabolic subalgebras of $\tilde{\mathfrak{l}}^{\prime \prime}$ and $\mathfrak{m}$ is a Levi subalgebra of both $\mathfrak{q}$ and $\mathfrak{q}^{\prime}$. We show:

(e) If $\mathfrak{H}=\mathfrak{H}_{\alpha_{0}, 2, \eta}$ for some $\left(\alpha_{0}, 2\right) \in \mathcal{R}_{\delta}^{*}$, then $I_{\varpi} \cong I_{\varpi^{\prime}}$ and $h(\varpi)=h\left(\varpi^{\prime}\right)$.

By (a) we have $\mathfrak{p}_{N} \subset \mathfrak{p}_{N}^{\prime \prime}, \mathfrak{p}_{N}^{\prime} \subset \mathfrak{p}_{N}^{\prime \prime}$ for all $N \in \mathbf{Z}$. Since $\mathfrak{H}_{\alpha_{0}, 2, \eta}$ is the unique hyperplane (as in 10.1) on which $\varpi^{\prime \prime}$ lies, we have $\varpi^{\prime \prime} \in \mathbf{E}^{\prime \prime}$. Hence by 10.3(b) we have $\tilde{\mathfrak{l}}_{N}^{\prime \prime} \subset \tilde{\mathfrak{l}}_{N}^{\phi}$ for all $N \in \mathbf{Z}$. In particular, the $\mathbf{Z}$-grading of $\tilde{\mathfrak{l}}^{\prime \prime}$ is $\eta$-rigid and $e \in \stackrel{\circ}{\mathfrak{l}}_{\eta}^{\prime \prime}$, so that $\stackrel{\circ}{\mathfrak{m}}{ }_{\eta} \subset \stackrel{\circ}{\mathfrak{l}}_{\eta}^{\prime \prime}$. Let $A \in \mathcal{Q}\left(\tilde{\mathfrak{l}}_{\eta}^{\prime \prime}\right)$ be the simple perverse sheaf on $\tilde{\mathfrak{l}}_{\eta}^{\prime \prime}$ such that the support of $A$ is $\tilde{\mathfrak{l}}_{\eta}^{\prime \prime}$ and $\left.A\right|_{\mathfrak{m}_{\eta}}$ is equal up to shift to $\left.\tilde{C}\right|_{\mathfrak{m}_{\eta}}$. Applying (twice) [LY, 1.8(b)] and the transitivity formula 4.2(a) we deduce

$$
\begin{aligned}
& { }^{\epsilon} \operatorname{Ind}_{\mathfrak{p}_{\eta}}^{\mathfrak{g}_{\delta}}(\tilde{C})={ }^{\epsilon} \operatorname{Ind}_{\mathfrak{p}_{\eta}^{\prime \prime}}^{\mathfrak{g}_{\delta}}\left(\operatorname{ind}_{\mathfrak{q}_{\eta}} \tilde{\mathfrak{l}}_{\eta}^{\prime \prime \prime}(\tilde{C})\right) \cong \oplus_{j}{ }^{\epsilon} \operatorname{Ind}_{\mathfrak{p}_{\eta}^{\prime \prime}}^{\mathfrak{g} \delta}(A)\left[-2 s_{j}\right]\left[\operatorname{dim} \mathfrak{m}_{\eta}-\operatorname{dim} \tilde{\mathfrak{l}}_{\eta}^{\prime \prime}\right], \\
& { }^{\epsilon} \operatorname{Ind}_{\mathfrak{p}_{\eta}^{\prime}}^{\mathfrak{g}_{\delta}}(\tilde{C})={ }^{\epsilon} \operatorname{Ind}_{\mathfrak{p}_{\eta}^{\prime \prime}}^{\mathfrak{g}_{\delta}}\left(\operatorname{ind}_{\mathfrak{q}_{\eta}^{\prime}} \tilde{\mathfrak{l}}_{\eta}^{\prime \prime}(\tilde{C})\right) \cong \oplus_{j}{ }^{\epsilon} \operatorname{Ind}_{\mathfrak{p}_{\eta}^{\prime \prime}}^{\mathfrak{g}_{\delta}}(A)\left[-2 s_{j}\right]\left[\operatorname{dim} \mathfrak{m}_{\eta}-\operatorname{dim} \tilde{\mathfrak{l}}_{\eta}^{\prime \prime \prime}\right],
\end{aligned}
$$

where $\left(s_{j}\right)$ is a certain finite collection of integers. It follows that

$$
{ }^{\epsilon} \operatorname{Ind}_{\mathfrak{p}_{\eta}}^{\mathfrak{g}_{\delta}}(\tilde{C}) \cong \operatorname{Ind}_{\mathfrak{p}_{\eta}^{\prime}}^{\mathfrak{g}_{\delta}}(\tilde{C}) .
$$

This shows that $I_{\varpi}, I_{\varpi^{\prime}}$ are isomorphic. It remains to show that $h(\varpi)=h\left(\varpi^{\prime}\right)$, for which it suffices to show the following two equalities:

$$
\begin{aligned}
\operatorname{dim} \mathfrak{u}_{0} & =\operatorname{dim} \mathfrak{u}_{0}^{\prime}, \\
\operatorname{dim} \mathfrak{u}_{\eta} & =\operatorname{dim} \mathfrak{u}_{\eta}^{\prime} .
\end{aligned}
$$

Since $\mathfrak{p}_{0}$ and $\mathfrak{p}_{0}^{\prime}$ are associate parabolic subalgebras of $\mathfrak{p}_{0}^{\prime \prime}$ sharing the same Levi subalgebra $\mathfrak{m}_{0}$, their unipotent radicals $\mathfrak{u}_{0}$ and $\mathfrak{u}_{0}^{\prime}$ have the same dimension. This proves (f).

Now we show (g). By 10.2(i), we have

$$
\operatorname{dim} \mathfrak{u}_{\eta}-\operatorname{dim} \mathfrak{u}_{\eta}^{\prime}=\sum_{\alpha \in X_{Z} ;\langle\varpi: \alpha\rangle>0,\left\langle\varpi^{\prime}: \alpha\right\rangle<0} \operatorname{dim} \mathfrak{g}_{\delta}^{\alpha, 2}-\sum_{\alpha \in X_{Z} ;\langle\varpi: \alpha\rangle<0,\left\langle\varpi^{\prime}: \alpha\right\rangle>0} \operatorname{dim} \mathfrak{g}_{\delta}^{\alpha, 2} .
$$

We want to show the above difference is zero. For this, it suffices to show that $\operatorname{dim} \mathfrak{g}_{\delta}^{\alpha, 2}=\operatorname{dim} \mathfrak{g}_{\delta}^{-\alpha, 2}$ for any $\alpha \in X_{Z}$. Note that $\mathfrak{g}_{\delta}^{\alpha, 2}=\tilde{\mathfrak{l}}_{\eta}^{\phi} \cap \mathfrak{g}^{\alpha}$, which is the $\alpha$-weight space of $Z$ on $\tilde{\mathfrak{l}}_{\eta}^{\phi}$. By the property of the $\mathfrak{s l}_{2}$-action on $\tilde{\mathfrak{l}}^{\phi}$ given by the 
triple $\phi=(e, h, f)$, we have that ad(e) induces an isomorphism $\tilde{\mathfrak{l}}_{-\eta}^{\phi} \cong \tilde{\mathfrak{l}}_{\eta}^{\phi}$. Since $Z$ commutes with $e$, this isomorphism restricts to an isomorphism of $\alpha$-weight spaces $\tilde{\mathfrak{l}}_{-\eta}^{\phi} \cap \mathfrak{g}^{\alpha} \cong \tilde{\mathfrak{l}}_{\eta}^{\phi} \cap \mathfrak{g}^{\alpha}$, that is, $\mathfrak{g}_{-\delta}^{\alpha,-2} \cong \mathfrak{g}_{\delta}^{\alpha, 2}$. Therefore $\operatorname{dim} \mathfrak{g}_{\delta}^{\alpha, 2}=\operatorname{dim} \mathfrak{g}_{-\delta}^{\alpha,-2}=$ $\operatorname{dim} \mathfrak{g}_{\delta}^{-\alpha, 2}$, as desired. This proves (g) and finishes the proof of (e).

10.7. Let

$$
\begin{aligned}
\stackrel{\circ}{\mathbf{E}} & =\mathbf{E}-\cup_{(\alpha, n) \in \mathcal{R}_{\delta}^{*} ; n \neq 2} \mathfrak{H}_{\alpha, n, \eta} \\
& =\left\{\varpi \in \mathbf{E} ;\langle\varpi: \alpha\rangle+n-2 \neq 0 \quad \forall(\alpha, n) \in \mathcal{R}_{\delta}^{*} \text { such that } n \neq 2\right\} .
\end{aligned}
$$

Note that $\mathbf{E}^{\prime} \subset \mathbf{E}^{\prime \prime} \subset \stackrel{\circ}{\mathbf{E}}$. For ${ }^{\prime} \varpi,{ }^{\prime \prime} \varpi$ in $\stackrel{\circ}{\mathbf{E}}$ we say that ${ }^{\prime} \varpi \sim " \varpi$ if for any $(\alpha, n) \in \mathcal{R}_{\delta}^{*}$ such that $n \neq 2$ we have

$$
\left(\left\langle^{\prime} \varpi: \alpha\right\rangle+n-2\right)\left(\left\langle^{\prime \prime} \varpi: \alpha\right\rangle+n-2\right)>0 .
$$

This is an equivalence relation on $\stackrel{\circ}{\mathbf{E}}$. We show:

(a) Assume that ${ }^{\prime} \varpi,{ }^{\prime \prime} \varpi$ in $\mathbf{E}^{\prime}$ satisfy ${ }^{\prime} \varpi \sim^{\prime \prime} \varpi$. Then $I_{\varpi} \cong I_{\prime \prime}$ and $h\left({ }^{\prime} \varpi\right)=$ $h\left({ }^{\prime \prime} \varpi\right)$; hence $\tilde{I}^{\prime} \varpi \simeq \tilde{I}_{\prime \prime}$.

By a known property of hyperplane arrangements, we can find a sequence $\varpi_{0}, \varpi_{1}$, $\ldots, \varpi_{k}$ in $\mathbf{E}^{\prime}$ such that $\varpi_{0}={ }^{\prime} \varpi, \varpi_{k}={ }^{\prime \prime} \varpi$ and such that for any $j \in\{0,1, \ldots, k-1\}$ one of the following holds:

$$
\begin{aligned}
& \varpi_{j} \equiv \varpi_{j+1} ; \\
& \varpi_{j}=\varpi, \varpi_{j+1}=\varpi^{\prime} \text { are as in } 10.6(\mathrm{c}) ; \\
& \varpi_{j}=\varpi, \varpi_{j+1}=\varpi^{\prime} \text { are as in } 10.6(\mathrm{~d}) ; \\
& \varpi_{j}=\varpi, \varpi_{j+1}=\varpi^{\prime} \text { are as in 10.6(e). }
\end{aligned}
$$

Using 10.2(l) or $10.6(\mathrm{c})$ or $10.6(\mathrm{~d})$ or $10.6(\mathrm{e})$ we see that for $j \in\{0,1, \ldots, k-1\}$ we have $I_{\varpi_{j}} \cong I_{\varpi_{j+1}}$ and $h\left(\varpi_{j}\right)=h\left(\varpi_{j+1}\right)$. This proves (a).

The set of equivalence classes on $\stackrel{\circ}{\mathbf{E}}$ for $\sim$ is denoted by $\stackrel{\circ}{\mathbf{E}}$; it is a finite set. 10.8. For $\varpi, \varpi^{\prime}$ in $\mathbf{E}$ we set

$$
\tau\left(\varpi, \varpi^{\prime}\right)=\sum_{(\alpha, n) \in \mathcal{R}_{\delta}^{*} ;(\langle\varpi: \alpha\rangle+n-2)\left(\left\langle\varpi^{\prime}: \alpha\right\rangle+n-2\right)<0} \operatorname{dim} \mathfrak{g}_{\delta}^{\alpha, n},
$$

$$
\sum_{(\alpha, n) \in \mathcal{R}_{\underline{0}}^{*} ;(\langle\varpi: \alpha\rangle+n)\left(\left\langle\varpi^{\prime}: \alpha\right\rangle+n\right)<0} \operatorname{dim} \mathfrak{g}_{\underline{0}}^{\alpha, n} .
$$

Using 10.2(i) we see that when $\varpi, \varpi^{\prime}$ are in $\mathbf{E}^{\prime}$, then

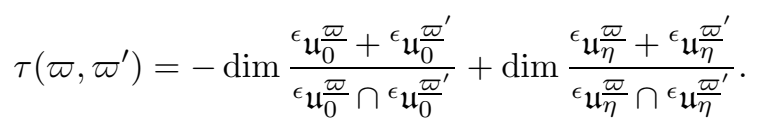

10.9. We define $G_{\phi}, M_{\phi}$ as in [LY, 3.6]. We show that:

(a) The obvious map $M_{\phi} / M_{\phi}^{0} \rightarrow\left(G_{\underline{0}} \cap G_{\phi}\right) /\left(G_{\underline{0}} \cap G_{\phi}\right)^{0}$ is an isomorphism.

Recall that $\phi=(e, h, f)$ with $e \in \stackrel{\circ}{\mathfrak{m}}_{\eta}$. Let $U$ (resp. $U^{\prime}$ ) be the unipotent radical of $G(e)^{0}$ (resp. $\left.(M \cap G(e))^{0}\right)$. We have $G(e)=G_{\phi} U$ (semidirect product). Taking fixed point sets of $\vartheta$ we obtain $G_{\underline{0}} \cap G(e)=\left(G_{\underline{0}} \cap G_{\phi}\right)\left(G_{\underline{0}} \cap U\right)$ (semidirect product). We have $M \cap G(e)=M_{\phi} U^{\prime}$ (semidirect product). Taking fixed point set of $\iota(t)$ for all $t \in \mathbf{k}^{*}$ we obtain $M_{0} \cap G(e)=\left(M_{0} \cap M_{\phi}\right)\left(M_{0} \cap U^{\prime}\right)=M_{\phi}\left(M_{0} \cap U^{\prime}\right)$ (semidirect product). (We have used that $M_{\phi} \subset M_{0}$.) It follows that we have canonically

$$
\left(M_{0} \cap G(e)\right) /\left(M_{0} \cap G(e)\right)^{0}=M_{\phi} / M_{\phi}^{0},
$$




$$
\left(G_{\underline{0}} \cap G(e)\right) /\left(G_{\underline{0}} \cap G(e)\right)^{0}=\left(G_{\underline{0}} \cap G_{\phi}\right) /\left(G_{\underline{0}} \cap G_{\phi}\right)^{0} .
$$

It remains to use that

$$
\left(M_{0} \cap G(e)\right) /\left(M_{0} \cap G(e)\right)^{0}=\left(G_{\underline{0}} \cap G(e)\right) /\left(G_{\underline{0}} \cap G(e)\right)^{0} ;
$$

see [LY, 3.8(a)].

Recall from [LY, 3.6] that $Z$ is a maximal torus of $\left(G_{\phi} \cap G_{0}\right)^{0}$. Let $H$ be the normalizer of $Z$ in $\left(G_{\phi} \cap G_{\underline{0}}\right)^{0}$. Let $H^{\prime}=\left\{g \in G_{\underline{0}} ; \operatorname{Ad}(g) M=M, \operatorname{Ad}(g) \mathfrak{m}_{k}=\right.$ $\left.\mathfrak{m}_{k} \forall k \in \mathbf{Z}\right\}$. Note that $M_{0}$ is a normal subgroup of $H^{\prime}$. Hence the groups $H / Z$, $H^{\prime} / M_{0}$ are defined. We show:

(b) $H \subset H^{\prime}$.

Let $g \in\left(G_{\phi} \cap G_{\underline{0}}\right)^{0}$ be such that $g Z g^{-1}=Z$. Let

$$
\left(M^{\prime}, M_{0}^{\prime}, \mathfrak{m}^{\prime}, \mathfrak{m}_{*}^{\prime}\right)=\left(g M g^{-1}, g M_{0} g^{-1}, \operatorname{Ad}(g) \mathfrak{m}, \operatorname{Ad}(g) \mathfrak{m}_{*}\right) .
$$

Note that $\mathcal{Z}_{M^{\prime}}^{0}=\mathcal{Z}_{M}^{0}=Z$. Repeating the argument in the last paragraph in the proof of [LY, 3.6(a)], we see that

$$
\left(M^{\prime}, M_{0}^{\prime}, \mathfrak{m}^{\prime}, \mathfrak{m}_{*}^{\prime}\right)=\left(M, M_{0}, \mathfrak{m}, \mathfrak{m}_{*}\right) .
$$

(The argument is applicable since $g \in G_{\phi} \cap G_{\underline{0}}$.) We see that $g \in H^{\prime}$; this proves (b).

We show:

(c) $H \cap M_{0}=Z$.

From the injectivity of the map in (a) we see that $M_{\phi} \cap\left(G_{\underline{0}} \cap G_{\phi}\right)^{0}=M_{\phi}^{0}$. Hence we have

$$
M_{0} \cap\left(G_{\underline{0}} \cap G_{\phi}\right)^{0} \subset\left(M_{0} \cap G_{\phi}\right) \cap\left(G_{\underline{0}} \cap G_{\phi}\right)^{0} \subset M_{\phi} \cap\left(G_{\underline{0}} \cap G_{\phi}\right)^{0}=M_{\phi}^{0},
$$

so that

$$
M_{0} \cap\left(G_{\underline{0}} \cap G_{\phi}\right)^{0} \subset M_{\phi}^{0} .
$$

The opposite inclusion is also true since $M_{\phi} \subset M_{0}$ and $M_{\phi}^{0} \subset G_{\underline{0}} \cap G_{\phi}$. It follows that

$$
M_{0} \cap\left(G_{\underline{0}} \cap G_{\phi}\right)^{0}=M_{\phi}^{0}=Z .
$$

The last equality is because $e$ is distinguished in $\mathfrak{m}$. Now $H \cap M_{0}$ is the normalizer of $Z$ in $M_{0} \cap\left(G_{\phi} \cap G_{\underline{0}}\right)^{0}$, that is, the normalizer of $Z$ in $Z$. We see that $H \cap M_{0}=Z$. This proves (c).

We show:

(d) $H^{\prime}=M_{0} H$.

Since $H \subset H^{\prime}, M_{0} \subset H^{\prime}$, we have $M_{0} H \subset H^{\prime}$. Now let $g \in H^{\prime}$. We show that $g \in M_{0} H$. Let $\phi^{\prime}=(\operatorname{Ad}(g) e, \operatorname{Ad}(g) h, \operatorname{Ad}(g) f)$. We have

$$
\operatorname{Ad}(g) e \in \stackrel{\circ}{\mathfrak{m}}_{\eta}, \operatorname{Ad}(g) h \in \mathfrak{m}_{0}, \operatorname{Ad}(g) f \in \mathfrak{m}_{-\eta} .
$$

Since both $\operatorname{Ad}(g) e, e$ are in $\stackrel{\circ}{\mathfrak{m}}_{\eta}$, we can find $g_{1} \in M_{0}$ such that $\operatorname{Ad}\left(g_{1}\right) \operatorname{Ad}(g) e=e$. Replacing $g$ by $g_{1} g$ we can assume that we have $\operatorname{Ad}(g) e=e$. Using [L4, 3.3] for $J^{M}$, we can find $g_{2} \in M_{0}$ such that

$$
\left(\operatorname{Ad}\left(g_{2}\right) \operatorname{Ad}(g) e, \operatorname{Ad}\left(g_{2}\right) \operatorname{Ad}(g) h, \operatorname{Ad}\left(g_{2}\right) \operatorname{Ad}(g) f\right)=(e, h, f) .
$$

We have $g_{2} g \in G_{\phi}$. Replacing $g$ by $g_{2} g$ we can assume that $g \in G_{\underline{0}} \cap G_{\phi}$. Using the surjectivity of the map in (a) we see that:

$$
G_{\underline{0}} \cap G_{\phi} \subset M_{\phi}\left(G_{\underline{0}} \cap G_{\phi}\right)^{0} .
$$


Thus we can write $g$ in the form $g_{3} g^{\prime}$ with $g_{3} \in M_{\phi}, g^{\prime} \in\left(G_{\underline{0}} \cap G_{\phi}\right)^{0}$. Replacing $g$ by $g_{3}^{-1} g$ we see that we can assume that $g \in\left(G_{\underline{0}} \cap G_{\phi}\right)^{0}$. Since $\operatorname{Ad}(g) M=M$, we see that $\operatorname{Ad}(g) Z=Z$. Thus $g \in H$. This proves (d).

From (b),(c),(d) we see that:

(e) The inclusion $H \subset H^{\prime}$ induces an isomorphism $H / Z \stackrel{\sim}{\rightarrow} H^{\prime} / M_{0}$. In particular, $M_{0}$ is the identity component of $H^{\prime}$.

10.10. Let $g \in H^{\prime}$ (notation of 10.9). Then $\operatorname{Ad}(g)$ restricts to an isomorphism $\mathfrak{m}_{\eta} \stackrel{\sim}{\rightarrow} \mathfrak{m}_{\eta}$. Let $\tilde{C}^{\prime}=\operatorname{Ad}(g)^{*} \tilde{C}$, a simple perverse sheaf in $\mathcal{Q}\left(\mathfrak{m}_{\eta}\right)$. We show:

(a) $\tilde{C}^{\prime} \cong \tilde{C}$.

Using $10.9(\mathrm{e})$ we can assume that $g \in H$ (notation of 10.9 ). Since $\tilde{C}, \tilde{C}^{\prime}$ are intersection cohomology complexes attached to $M_{0}$-equivariant irreducible local systems on $\mathfrak{m}_{\eta}$, they correspond to irreducible representations of

$$
\left(M_{0} \cap G(e)\right) /\left(M_{0} \cap G(e)\right)^{0}=M_{\phi} / M_{\phi}^{0} .
$$

Hence it is enough to show that $\operatorname{Ad}(g)$ induces the identity automorphism of $M_{\phi} / M_{\phi}^{0}$. Using $10.9(\mathrm{a})$, we see that it is enough to show that $\operatorname{Ad}(g)$ induces the identity automorphism of $\left(G_{\underline{0}} \cap G_{\phi}\right) /\left(G_{\underline{0}} \cap G_{\phi}\right)^{0}$. This is obvious since $g \in$ $\left(G_{\underline{0}} \cap G_{\phi}\right)^{0}$. This proves (a).

10.11. Let $\varpi, \varpi^{\prime} \in \mathbf{E}^{\prime}$. Recall that $P_{0}=e^{\epsilon \mathfrak{p}_{0}^{\frac{\varpi}{0}}} \subset G_{0}, P_{0}^{\prime}=e^{\epsilon \mathfrak{p}_{0}^{\varpi^{\prime}}} \subset G_{0}$ are parabolic subgroups of $G_{\underline{0}}$ with a common Levi subgroup $M_{0}$. Let $U_{0}=U_{P_{0}}, \bar{U}_{0}^{\prime}=U_{P_{0}^{\prime}}$. Let $X$ be the set of all $g \in G_{\underline{0}}$ such that $\operatorname{Ad}(g) \mathfrak{p}_{*}^{\varpi}$ and $\mathfrak{p}_{*}^{\varpi^{\prime}}$ have a common splitting (as in 6.3). Note that $X$ is a union of $\left(P_{0}^{\prime}, P_{0}\right)$-double cosets in $G_{\underline{0}}$. We show:

(a) We have $H^{\prime} \subset X$ (notation of 10.9). Let $j: H^{\prime} / M_{0} \rightarrow P_{0}^{\prime} \backslash X / P_{0}$ be the map induced by the inclusion $H^{\prime} \rightarrow X$. Then $j$ is a bijection.

If $g \in H^{\prime}$, then $g \in G_{\underline{0}}$ and $\operatorname{Ad}(g) \mathfrak{m}_{*}=\mathfrak{m}_{*}$ hence $\mathfrak{m}_{*}$ is a common splitting of $\operatorname{Ad}(g) \mathfrak{p}_{*}^{\varpi}$ and $\mathfrak{p}_{*}^{\varpi^{\prime}}$. Thus we have $g \in X$, so that the inclusion $H^{\prime} \subset X$ holds. Let $g \in$ $X$. Then $g \in G_{\underline{0}}$ and $\operatorname{Ad}(g) \mathfrak{p}_{*}^{\varpi}, \mathfrak{p}_{*}^{\varpi^{\prime}}$ have a common splitting $\mathfrak{m}_{*}^{\prime}$. Then $\operatorname{Ad}(g) \mathfrak{m}_{*}, \mathfrak{m}_{*}^{\prime}$ are splittings of $\operatorname{Ad}(g) \mathfrak{p}_{*}^{\varpi}$ hence, by [LY, 2.7(a)], we have $\operatorname{Ad}\left(g u g^{-1}\right) \operatorname{Ad}(g) \mathfrak{m}_{*}=\mathfrak{m}_{*}^{\prime}$ for some $u \in U_{0}$. Moreover, $\mathfrak{m}_{*}, \mathfrak{m}_{*}^{\prime}$ are splittings of $\mathfrak{p}_{*}^{\varpi^{\prime}}$ hence, by [LY, 2.7(a)], we have $\operatorname{Ad}\left(u^{\prime}\right) \mathfrak{m}_{*}=\mathfrak{m}_{*}^{\prime}$ for some $u^{\prime} \in U_{0}^{\prime}$. It follows that $\operatorname{Ad}(g u) \mathfrak{m}_{*}=\operatorname{Ad}\left(u^{\prime}\right) \mathfrak{m}_{*}$ hence $u^{\prime-1} g u \in H^{\prime}$. Since $U_{0} \subset P_{0}, U_{0}^{\prime} \subset P_{0}^{\prime}$, we see that $j$ is surjective. It remains to show that $j$ is injective. Let $g, g^{\prime}$ be elements of $H^{\prime}$ such that $g^{\prime}=p_{0}^{\prime} g p_{0}$ for some $p_{0} \in P_{0}, p_{0}^{\prime} \in P_{0}^{\prime}$. We must only show that $g^{\prime} \in g M_{0}$. Let $N M_{0}$ be the normalizer of $M_{0}$ in $G_{\underline{0}}$. It is enough to show that the obvious map $N M_{0} / M_{0} \rightarrow P_{0}^{\prime} \backslash G_{\underline{0}} / P_{0}$ is injective. This is a well-known property of parabolic subgroups and their Levi subgroups in a connected reductive group. This completes the proof of (a).

Let $\mathcal{W}=H / Z$ (notation of 10.9 ). Let $w \in \mathcal{W}$ and let $g \in H$ be a representative of $w$. Now $\operatorname{Ad}(g)$ restricts to an automorphism of $Z$ which depends only on $w$; this induces an isomorphism $Y_{Z} \stackrel{\sim}{\rightarrow} Y_{Z}$ and, by extension of scalars, a vector space isomorphism $\mathbf{E} \stackrel{\sim}{\longrightarrow} \mathbf{E}$ denoted by $\varpi_{1} \mapsto w \varpi_{1}$. For any $(\alpha, n, i) \in X_{Z} \times \mathbf{Z} \times \mathbf{Z} / m$, $\operatorname{Ad}(g)$ defines an isomorphism $\mathfrak{g}_{i}^{\alpha, n} \stackrel{\sim}{\rightarrow} \mathfrak{g}_{i}{ }^{w}{ }^{\alpha, n}$ where ${ }^{w} \alpha \in X_{Z}$ is given by ${ }^{w} \alpha(z)=$ $\alpha\left(w^{-1}(z)\right)$; hence for any $i,(\alpha, n) \mapsto\left({ }^{w} \alpha, n\right)$ is a bijection $\mathcal{R}_{i} \stackrel{\sim}{\rightarrow} \mathcal{R}_{i}$. Moreover, for any $N \in \mathbf{Z}$ and any $(\alpha, n) \in \mathcal{R}_{N}^{*}, w: \mathbf{E} \rightarrow \mathbf{E}$ restricts to a bijection from the affine hyperplane $\mathfrak{H}_{\alpha, n, N}$ to the affine hyperplane $\mathfrak{H}_{w_{\alpha, n, N}}$. It follows that $w: \mathbf{E} \rightarrow \mathbf{E}$ restricts to a bijection $\mathbf{E}^{\prime} \stackrel{\sim}{\rightarrow} \mathbf{E}^{\prime}$. 
We show:

(b) For any $\varpi \in \mathbf{E}^{\prime}$ we have $\operatorname{Ad}(g)\left({ }^{\epsilon} \mathfrak{p}_{*}^{\varpi}\right)={ }^{\epsilon} \mathfrak{p}^{w}{ }^{w \varpi}$.

From 10.2(h) we have

$$
\begin{aligned}
& \operatorname{Ad}(g)\left({ }^{\epsilon} \mathfrak{p}^{\frac{\varpi}{N}}\right)=\oplus_{(\alpha, n) \in \mathcal{R}_{\underline{N}} ;\langle\varpi: \alpha\rangle \geq 2 N / \eta-n} \operatorname{Ad}(g) \mathfrak{g}_{\underline{N}}^{\alpha, n} \\
& =\oplus_{(\alpha, n) \in \mathcal{R}_{\underline{N}} ;\langle\varpi: \alpha\rangle \geq 2 N / \eta-n}\left(\mathfrak{g}_{\underline{N}}^{w} \alpha, n\right), \\
& \epsilon_{\mathfrak{p}} \frac{w \varpi}{N}=\oplus_{(\alpha, n) \in \mathcal{R}_{\underline{N}} ;\langle w \varpi: \alpha\rangle \geq 2 N / \eta-n}\left(\mathfrak{g}_{\underline{N}}^{\alpha, n}\right) \\
& =\oplus_{\left(\alpha^{\prime}, n\right) \in \mathcal{R}_{\underline{N}} ;\left\langle w \varpi:{ }^{w} \alpha^{\prime}\right\rangle \geq 2 N / \eta-n}\left(\mathfrak{g}_{\underline{N}}^{w} \alpha^{\prime}, n\right) .
\end{aligned}
$$

It remains to use that $\langle\varpi: \alpha\rangle=\left\langle w \varpi:{ }^{w} \alpha\right\rangle$.

10.12. For $\varpi_{1}, \varpi_{2}$ in $\mathbf{E}$ we set

$$
\left[\varpi_{1} \mid \varpi_{2}\right]=\left(1-v^{2}\right)^{-\operatorname{dim} Z} \sum_{w \in \mathcal{W}} v^{\tau\left(\varpi_{2}, w \varpi_{1}\right)} \in \mathbf{Q}(v) .
$$

(Here $v$ is an indeterminate and $\tau\left(\varpi_{2}, w \varpi_{1}\right) \in \mathbf{Z}$ is as in 10.8.) When $\varpi_{1}, \varpi_{2}$ are in $\mathbf{E}^{\prime}$ we have:

$$
\sum_{j \in \mathbf{Z}} d_{j}\left(\mathfrak{g}_{\delta} ; \tilde{I}_{\varpi_{1}}, D\left(\tilde{I}_{\varpi_{2}}\right)\right) v^{-j}=\left[\varpi_{1} \mid \varpi_{2}\right]
$$

This can be deduced from [LY, 6.4] as follows. The set $X$ in [LY, 6.3] is described in our case in 10.11(a) in terms of the group $H^{\prime} / M_{0}$ which, in turn, is identified in $10.9(\mathrm{e})$ with $\mathcal{W}=H / Z$; the integers $\tau(g)$ in 6.3 are identified with the integers $\tau\left(\varpi_{2}, w \varpi_{1}\right) \in \mathbf{Z}$ by $10.11(\mathrm{~b})$. Finally, the set $X^{\prime}$ in [LY, 6.4] coincides with $X$ in [LY, 6.4] by $10.10(\mathrm{a})$.

From the definitions we have $\tau\left(\varpi_{2}, w \varpi_{1}\right)=\tau\left(w^{-1} \varpi_{2}, \varpi_{1}\right)=\tau\left(\varpi_{1}, w^{-1} \varpi_{2}\right)$ for any $w \in \mathcal{W}$. It follows that

$$
\left[\varpi_{1} \mid \varpi_{2}\right]=\left[\varpi_{2} \mid \varpi_{1}\right]
$$

Let $\mathbf{c}_{1}$ (resp. $\mathbf{c}_{2}$ ) be the equivalence class for $\sim$ in $\stackrel{\circ}{\mathbf{E}}$ that contains $\varpi_{1}$ (resp. $\varpi_{2}$ ). Using 10.7(a) we see that the right hand side of (b) depends only on $\mathbf{c}_{1}, \mathbf{c}_{2}$ and not on the specific elements $\varpi_{1} \in \mathbf{c}_{1} \cap \mathbf{E}^{\prime}, \varpi_{2} \in \mathbf{c}_{2} \cap \mathbf{E}^{\prime}$. Hence for $\mathbf{c}_{1}, \mathbf{c}_{2}$ in $\underline{\stackrel{\circ}{\mathbf{E}}}$ we can set $\left[\mathbf{c}_{1} \mid \mathbf{c}_{2}\right]=\left[\varpi_{1} \mid \varpi_{2}\right] \in \mathbf{Q}(v)$ for any $\varpi_{1} \in \mathbf{c}_{1} \cap \mathbf{E}^{\prime}, \varpi_{2} \in \mathbf{c}_{2} \cap \mathbf{E}^{\prime}$.

10.13. Let $\mathfrak{B}$ (resp. $\xi_{\mathfrak{B}}$ ) be the set of (isomorphism classes of) simple perverse sheaves in $\mathcal{Q}\left(\mathfrak{g}_{\delta}\right)$ (resp. $\left.{ }^{\xi} \mathcal{Q}\left(\mathfrak{g}_{\delta}\right)\right)$. For any $G_{\underline{0}}$-orbit $\mathcal{O}$ in $\mathfrak{g}_{\delta}^{\text {nil }}$ let $\mathfrak{B}_{\mathcal{O}}$ be the set of all $B \in \mathfrak{B}$ such that the support of $B$ is equal to the closure of $\mathcal{O}$. We have $\mathfrak{B}=\sqcup_{\mathcal{O}} \mathfrak{B}_{\mathcal{O}}$. We define a map $\kappa: \mathfrak{B} \rightarrow \mathbf{N}$ by $\kappa(B)=\operatorname{dim} \mathcal{O}$ where $B \in \mathfrak{B}_{\mathcal{O}}$.

10.14. Let $\mathbf{V}^{\prime}$ be the $\mathbf{Q}(v)$-vector space with basis $\left\{\tilde{T}_{\mathbf{c}} ; \mathbf{c} \in \stackrel{\circ}{\mathbf{E}}\right\}$. On $\mathbf{V}^{\prime}$ we have a unique pairing (:) : $\mathbf{V}^{\prime} \times \mathbf{V}^{\prime} \rightarrow \mathbf{Q}(v)$ which is $\mathbf{Q}(v)$-linear in the first argument, $\mathbf{Q}(v)$-antilinear in the second argument (for $f \mapsto \bar{f}$ ) and such that for $\mathbf{c}_{1}, \mathbf{c}_{2}$ in $\underline{\mathrm{E}}$ we have $\left(\tilde{T}_{\mathbf{c}_{1}}: \tilde{T}_{\mathbf{c}_{2}}\right)=\left[\mathbf{c}_{1} \mid \mathbf{c}_{2}\right]$ (see 10.12).

Setting

$$
\begin{array}{ll}
\mathfrak{R}_{l}=\left\{x \in \mathbf{V}^{\prime} ;\left(x: x^{\prime}\right)=0\right. & \left.\forall x^{\prime} \in \mathbf{V}^{\prime}\right\}, \\
\mathfrak{R}_{r}=\left\{x \in \mathbf{V}^{\prime} ;\left(x^{\prime}: x\right)=0\right. & \left.\forall x^{\prime} \in \mathbf{V}^{\prime}\right\},
\end{array}
$$

we state the following. 
Lemma 10.15. We have $\mathfrak{R}_{l}=\mathfrak{R}_{r}$.

The proof is given in 10.17 .

10.16. We define a Q-linear map

$$
\tilde{\gamma}: \mathbf{V}^{\prime} \rightarrow \mathbf{Q}(v) \otimes_{\mathcal{A}}{ }^{\xi} \mathcal{K}\left(\mathfrak{g}_{\delta}\right)
$$

by $\tilde{T}_{\mathbf{c}} \mapsto \tilde{I}_{\varpi}$, where $\varpi$ is an element of $\mathbf{c} \cap \mathbf{E}^{\prime}$. Now $\tilde{\gamma}$ is well-defined by $10.7(\mathrm{a})$ and is surjective by $[\mathrm{LY}, 8.4(\mathrm{~b})], 10.4(\mathrm{~b})$. We define a pairing

$$
\left(\mathbf{Q}(v) \otimes_{\mathcal{A}} \mathcal{K}\left(\mathfrak{g}_{\delta}\right)\right) \times\left(\mathbf{Q}(v) \otimes_{\mathcal{A}} \mathcal{K}\left(\mathfrak{g}_{\delta}\right)\right) \rightarrow \mathbf{Q}((v))
$$

(denoted by (:)) by requiring that it is $\mathbf{Q}(v)$-linear in the first argument, $\mathbf{Q}(v)$ antilinear in the second argument (for $f \mapsto \bar{f}$ ) and that its restriction

$$
\mathcal{K}\left(\mathfrak{g}_{\delta}\right) \times \mathcal{K}\left(\mathfrak{g}_{\delta}\right) \rightarrow \mathbf{Q}((v))
$$

is the same as the restriction of the pairing in $[\mathrm{LY}, 4.4(\mathrm{c})]$. This restricts to a pairing

$$
\left(\mathbf{Q}(v) \otimes_{\mathcal{A}}{ }^{\xi} \mathcal{K}\left(\mathfrak{g}_{\delta}\right)\right) \times\left(\mathbf{Q}(v) \otimes_{\mathcal{A}}{ }^{\xi} \mathcal{K}\left(\mathfrak{g}_{\delta}\right)\right) \rightarrow \mathbf{Q}((v))
$$

(denoted again by $(:)$ ). We show that for $b, b^{\prime}$ in $\mathbf{V}^{\prime}$ we have

$$
\left(\tilde{\gamma}(b): \tilde{\gamma}\left(b^{\prime}\right)\right)=\left(b: b^{\prime}\right)
$$

We can assume that $b=\tilde{T}_{\mathbf{c}}, b^{\prime}=\tilde{T}_{\mathbf{c}^{\prime}}$ with $\mathbf{c}, \mathbf{c}^{\prime}$ in $\underline{\mathbf{E}}$. We must show that $\left\{\tilde{I}_{\varpi}, D\left(\tilde{I}_{\varpi^{\prime}}\right)\right\}$ $=\left[\varpi \mid \varpi^{\prime}\right]$ where $\varpi \in \mathbf{c} \cap \mathbf{E}^{\prime}, \varpi^{\prime} \in \mathbf{c}^{\prime} \cap \mathbf{E}^{\prime}$. This follows from 10.12(b). This proves (a).

10.17. Let

$$
\begin{aligned}
& { }^{\prime} \mathfrak{R}_{l}=\left\{z \in \mathbf{Q}(v) \otimes_{\mathcal{A}}{ }^{\xi} \mathcal{K}\left(\mathfrak{g}_{\delta}\right) ;\left(z: z^{\prime}\right)=0 \quad \forall z^{\prime} \in \mathbf{Q}(v) \otimes_{\mathcal{A}}{ }^{\xi} \mathcal{K}\left(\mathfrak{g}_{\delta}\right)\right\}, \\
& { }^{\prime} \mathfrak{R}_{r}=\left\{z \in \mathbf{Q}(v) \otimes_{\mathcal{A}}{ }^{\xi} \mathcal{K}\left(\mathfrak{g}_{\delta}\right) ;\left(z^{\prime}: z\right)=0 \quad \forall z^{\prime} \in \mathbf{Q}(v) \otimes_{\mathcal{A}}{ }^{\xi} \mathcal{K}\left(\mathfrak{g}_{\delta}\right)\right\} .
\end{aligned}
$$

We show:

(a) ${ }^{\prime} \mathfrak{R}_{l}={ }^{\prime} \mathfrak{R}_{r}=0$.

Now $\mathbf{Q}(v) \otimes_{\mathcal{A}} \xi \mathcal{K}\left(\mathfrak{g}_{\delta}\right)$ has a $\mathbf{Q}(v)$-basis formed by $\xi \mathfrak{B}=\left\{B_{1}, B_{2}, \ldots, B_{r}\right\}$. From [LY, 0.12] we see that $\left(B_{j}: B_{j^{\prime}}\right) \in \delta_{j, j^{\prime}}+v \mathbf{N}[[v]]$ for $j, j^{\prime}$ in $[1, r]$.

Assume that $\beta=\sum_{j \in[1, r]} f_{j} B_{j} \in{ }^{\prime} \mathfrak{R}_{l}$, where $f_{j} \in \mathbf{Q}(v)$ are not all zero. We must show that this is a contradiction. We can assume that $f_{j} \in \mathbf{Q}[v]$ for all $j$ and $f_{j_{0}}-c_{0} \in v \mathbf{Q}[v]$ for some $j_{0} \in[1, r]$ and some $c_{0} \in \mathbf{Q}-\{0\}$. Then $0=\left(\beta: B_{j_{0}}\right) \in$ $c_{0}+v \mathbf{Q}[[v]]$, a contradiction. This proves that ${ }^{\prime} \mathfrak{R}_{l}=0$.

Next we assume that $\beta=\sum_{j \in[1, r]} f_{j} B_{j} \in{ }^{\prime} \mathfrak{R}_{r}$, where $f_{j} \in \mathbf{Q}(v)$ are not all zero. We must show that this is a contradiction. We can assume that $\bar{f}_{j} \in \mathbf{Q}[v]$ for all $j$ and $\bar{f}_{j_{0}}-c_{0} \in v \mathbf{Q}[v]$ for some $j_{0} \in[1, r]$ and some $c_{0} \in \mathbf{Q}-\{0\}$. Then $0=\left(B_{j_{0}}: \beta\right) \in c_{0}+v \mathbf{Q}[[v]]$, a contradiction. This proves that ${ }^{\prime} \mathfrak{R}_{r}=0$. This proves (a).

We show:

(b) $\mathfrak{R}_{l}=\tilde{\gamma}^{-1}\left({ }^{\prime} \mathfrak{R}_{l}\right)$.

Let $x \in \mathfrak{R}_{l}$. From $10.16\left(\right.$ a) we see that $\left(\tilde{\gamma}(x): \tilde{\gamma}\left(x^{\prime}\right)\right)=0$ for any $x^{\prime} \in \mathbf{V}^{\prime}$. Since $\tilde{\gamma}$ is surjective, it follows that $\left(\tilde{\gamma}(x): z^{\prime}\right)=0$ for any $z^{\prime} \in \mathbf{Q}(v) \otimes_{\mathcal{A}} \xi \mathcal{K}\left(\mathfrak{g}_{\delta}\right)$. Thus, $\tilde{\gamma}(x) \in{ }^{\prime} \mathfrak{R}_{l}$. Conversely, assume that $x \in \mathbf{V}^{\prime}$ and $\tilde{\gamma}(x) \in{ }^{\prime} \mathfrak{R}_{l}$. From 10.16(a) we see that for any $x^{\prime} \in \mathbf{V}^{\prime}$ we have $\left(x: x^{\prime}\right)=\left(\tilde{\gamma}(x): \tilde{\gamma}\left(x^{\prime}\right)\right)=0$. Thus $x \in \mathfrak{R}_{l}$. This proves (b). 
An entirely similar proof shows that:

(c) $\mathfrak{R}_{r}=\tilde{\gamma}^{-1}\left({ }^{\prime} \mathfrak{R}_{r}\right)$.

From (a),(b),(c) we see that $\mathfrak{R}_{l}=\mathfrak{R}_{r}=\tilde{\gamma}^{-1}(0)$. This proves Lemma 10.15 .

10.18. Definition of $\mathbf{V}$. We define $\mathbf{V}=\mathbf{V}^{\prime} / \mathfrak{R}_{l}=\mathbf{V}^{\prime} / \mathfrak{R}_{r}$ (see Lemma 10.15). Note that (:) on $\mathbf{V}^{\prime}$ induces a pairing $\mathbf{V} \times \mathbf{V} \rightarrow \mathbf{Q}(v)$ (denoted again by (:)) which is $\mathbf{Q}(v)$-linear in the first argument, $\mathbf{Q}(v)$-antilinear in the second argument (for $f \mapsto \bar{f})$.

10.19. From the proof of Lemma 10.15 we see that $\tilde{\gamma}$ induces a $\mathbf{Q}(v)$-linear isomorphism

$$
\gamma: \mathbf{V} \stackrel{\sim}{\rightarrow} \mathbf{Q}(v) \otimes_{\mathcal{A}}{ }^{\xi} \mathcal{K}\left(\mathfrak{g}_{\delta}\right)
$$

and that for $b, b^{\prime}$ in $\mathbf{V}$ we have

$$
\left(\gamma(b): \gamma\left(b^{\prime}\right)\right)=\left(b: b^{\prime}\right) .
$$

From (a) we deduce the following result.

Proposition 10.20. The number of simple perverse sheaves (up to isomorphism) in $\xi \mathcal{Q}\left(\mathfrak{g}_{\delta}\right)$ is equal to $\operatorname{dim}_{\mathbf{Q}(v)} \mathbf{V}$.

10.21. We define a $\mathbf{Q}$-linear involution ${ }^{-}: \mathbf{V}^{\prime} \rightarrow \mathbf{V}^{\prime}$ by $\overline{f \tilde{T}_{\mathbf{c}}}=\bar{f} \tilde{T}_{\mathbf{c}}$ for any $f \in \mathbf{Q}(v)$, $\mathbf{c} \in \underline{\mathrm{E}}$; here $\bar{f}$ is as in [LY, 0.12]. We show:

(a) For any $x, x^{\prime}$ in $\mathbf{V}^{\prime}$ we have $\left(x: x^{\prime}\right)=\left(\bar{x}^{\prime}: \bar{x}\right)$.

We can assume that $x=\tilde{T}_{\mathbf{c}}, x^{\prime}=\tilde{T}_{\mathbf{c}^{\prime}}$ for some $\mathbf{c}, \mathbf{c}^{\prime}$ in $\stackrel{\circ}{\mathbf{E}}$. We must show that

$$
\left[\mathbf{c} \mid \mathbf{c}^{\prime}\right]=\left[\mathbf{c}^{\prime} \mid \mathbf{c}\right] \text {, }
$$

which follows directly from $10.12(\mathrm{c})$. This proves (a).

We show:

$(\mathrm{b})^{-}: \mathbf{V}^{\prime} \rightarrow \mathbf{V}^{\prime}$ preserves $\mathfrak{R}_{l}=\mathfrak{R}_{r}$ (see Lemma 10.15) hence it induces an $\mathbf{Q}(v)$-semilinear involution ${ }^{-}: \mathbf{V} \rightarrow \mathbf{V}$ (with respect to $f \mapsto \bar{f}$ ).

Assume that $x \in \mathfrak{R}_{l}$. We have $\left(x: x^{\prime}\right)=0$ for all $x^{\prime} \in \mathbf{V}$. Hence, by (a), we have $\left(\bar{x}^{\prime}: \bar{x}\right)=0$ for all $x^{\prime} \in \mathbf{V}$. Since ${ }^{-}: \mathbf{V}^{\prime} \rightarrow \mathbf{V}^{\prime}$ is surjective, it follows that $\bar{x} \in \mathfrak{R}_{r}$. This proves (b).

10.22. Now let $\eta_{1} \in \mathbf{Z}-\{0\}$ be such that $\underline{\eta}_{1}=\delta$. Recall that in 10.1 we have fixed $\xi \in \underline{\mathfrak{T}}_{\eta}$ and a representative $\dot{\xi}=\left(M, M_{0}, \mathfrak{m}, \mathfrak{m}_{*}, \tilde{C}\right) \in \mathfrak{T}_{\eta}$ for $\xi$. Let $\dot{\xi}_{1}=$ $\left(M, M_{0}, \mathfrak{m}, \mathfrak{m}_{(*)}, \tilde{C}\right) \in \mathfrak{T}_{\eta_{1}}$ be as in [LY, 3.9] and let $\xi_{1} \in \underline{\mathfrak{T}}_{\eta_{1}}$ be the $G_{\underline{0}^{-}}$orbit of $\dot{x}_{1}$. Note that the $\mathbf{Q}$-vector space $\mathbf{E}$ defined as in 10.1 in terms of $\dot{\xi}$ is the same as $\mathbf{E}$ defined in terms of $\dot{\xi}_{1}$. Moreover, the subset $\stackrel{\circ}{\mathbf{E}}$, the equivalence relation $\sim$ on it, and the set $\stackrel{\circ}{\mathbf{E}}$ defined as in 10.7 in terms of $\dot{\xi}$ are the same as the analogous objects defined in terms of $\dot{\xi}_{1}$. Also, the pairings $\tau: \mathbf{E} \times \mathbf{E} \rightarrow \mathbf{Z}$ and [?|?] $: \mathbf{E} \times \mathbf{E} \rightarrow \mathbf{Q}(v)$ defined in 10.8 and 10.12 in terms of $\dot{\xi}$ are the same as those defined in terms of $\dot{\xi}_{1}$.

The subset $\mathbf{E}^{\prime}$ of $\mathbf{E}$ defined as in 10.1 in terms of $\dot{\xi}$ is not in general the same as the analogous subset $\mathbf{E}_{1}^{\prime}$ of $\mathbf{E}$ defined in terms of $\dot{\xi}_{1}$. However, for $\mathbf{c}_{1}, \mathbf{c}_{2}$ in $\stackrel{\circ}{\mathbf{E}}$, the quantity $\left[\mathbf{c}_{1} \mid \mathbf{c}_{2}\right] \in \mathbf{Q}(v)$ defined in 10.12 in terms of $\dot{\xi}$ is the same as that defined in terms of $\dot{\xi}_{1}$. (It is equal to $\left[\varpi_{1} \mid \varpi_{2}\right]$ for any $\varpi_{1} \in \mathbf{c}_{1} \cap \mathbf{E}^{\prime} \cap \mathbf{E}_{1}^{\prime}, \varpi_{2} \in \mathbf{c}_{2} \cap \mathbf{E}^{\prime} \cap \mathbf{E}_{1}^{\prime}$. Hence the vector space $\mathbf{V}^{\prime}$, the pairing (:) on it and its quotient $\mathbf{V}$ defined in 10.14 
and 10.18 in terms of $\dot{\xi}$ are the same as those defined in terms of $\dot{\xi}_{1}$. The involutions ${ }^{-}: \mathbf{V}^{\prime} \rightarrow \mathbf{V}^{\prime},{ }^{-}: \mathbf{V} \rightarrow \mathbf{V}$ defined in 10.21 in terms of $\dot{\xi}$ are the same as those defined in terms of $\dot{\xi}_{1}$.

\section{The $\mathcal{A}$-Lattice $\mathbf{V}_{\mathcal{A}}$}

In this section we give a combinatorial definition of an $\mathcal{A}$-lattice $\mathbf{V}_{\mathcal{A}}$ in the $\mathbf{Q}(v)$ vector space $\mathbf{V}$ and a signed basis $\mathbf{B}^{\prime}$ of it. It turns out that the $\mathbf{Z} / 2$-orbits on $\mathbf{B}^{\prime}$ for the $\mathbf{Z} / 2$-action $b \mapsto-b$ are in natural bijection with the simple perverse sheaves in the block $\xi \mathcal{Q}\left(\mathfrak{g}_{\delta}\right)$.

11.1. In this section (except in 11.5, 11.6, 11.13) we preserve the setup and notation of 10.1, 10.2 and assume that $\varpi \in \mathbf{E}^{\prime \prime}$ (see 10.3). Let

$$
\mathfrak{g}^{\phi}=\{y \in \mathfrak{g} ;[y, e]=0,[y, h]=0,[y, f]=0\} .
$$

Let $\mathfrak{z}$ be the center of $\mathfrak{m}$. Note that $\mathfrak{m} \cap \mathfrak{g}^{\phi}=\mathfrak{z}$ (since $e$ is distinguished in $\mathfrak{m}$ ) and $\mathfrak{z}$ is a Cartan subalgebra of the reductive Lie algebra $\mathfrak{g}^{\phi}$. (This has already been proved at level of groups in [LY, 3.6].) For any $\alpha \in X_{Z}$ let

$$
\left(\mathfrak{g}_{\underline{0}}^{\phi}\right)^{\alpha}=\mathfrak{g}_{\underline{0}}^{\alpha} \cap \mathfrak{g}^{\phi} .
$$

Let

$$
\mathfrak{g}_{\underline{0}, \varpi}^{\phi}=\oplus_{\alpha \in X_{Z} ;\langle\varpi: \alpha\rangle=0}\left(\mathfrak{g}_{\underline{0}}^{\phi}\right)^{\alpha} .
$$

This is a Levi subalgebra (containing $\mathfrak{z}$ ) of a parabolic subalgebra of $\mathfrak{g}_{\underline{0}}^{\phi}$. Let $\mathcal{B}$ be the variety of Borel subalgebras of $\mathfrak{g}_{\underline{0}, \varpi}^{\phi}$, let $d(\varpi)=\operatorname{dim} \mathcal{B}$ and let

$$
a_{\varpi}=\sum_{j} v^{-2 s_{j}} v^{d(\varpi)} \in \mathcal{A},
$$

where $\rho_{\mathcal{B} !} \overline{\mathbf{Q}}_{l}=\oplus_{j} \overline{\mathbf{Q}}_{l}\left[-2 s_{j}\right]$.

Erratum to L4. On page 202, line 1 of 16.8 , replace "algebraic group $M$ " by "algebraic group $M$ with a given Lie algebra homomorphism $\phi$ from $\mathfrak{s}$ to the Lie algebra of $M$ ".

On page 202, line 4 of 16.8 , replace "Borel subgroups of $M$ " by "Borel subgroups of the connected centralizer of $\phi(\mathfrak{s})$ in $M$ ".

11.2. The subset

$$
\left\{a_{\varpi}^{-1} \tilde{T}_{\mathbf{c}} \in \mathbf{V}^{\prime} ; \mathbf{c} \in \stackrel{\circ}{\mathbf{E}}, \varpi \in \mathbf{E}^{\prime \prime} \cap \mathbf{c}\right\}
$$

of $\mathbf{V}^{\prime}$ (see 10.14) is finite. Indeed, when $\mathbf{c}$ is fixed, the subgroup $\mathfrak{g}_{\underline{0}, \varpi}^{\phi}$ of $\mathfrak{g}_{\underline{0}}^{\phi}$ takes only finitely many values for $\varpi$ in $\mathbf{E}^{\prime \prime} \cap \mathbf{c}$ hence $a_{\varpi}$ takes only finitely many values.

Let $\mathbf{V}_{\mathcal{A}}^{\prime}$ be the $\mathcal{A}$-submodule of $\mathbf{V}^{\prime}$ generated by (a). Let $\mathbf{V}_{\mathcal{A}}$ be the image of $\mathbf{V}_{\mathcal{A}}^{\prime}$ under the obvious linear map $\mathbf{V}^{\prime} \rightarrow \mathbf{V}$. The following result will be proved in 11.8 .

(b) $\mathbf{V}_{\mathcal{A}}$ is a free $\mathcal{A}$-module such that the obvious $\mathbf{Q}(v)$-linear map $\mathbf{Q}(v) \otimes_{\mathcal{A}} \mathbf{V}_{\mathcal{A}} \rightarrow$ $\mathbf{V}$ is an isomorphism. 


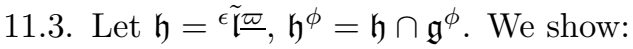

$$
\mathfrak{g}_{\underline{0}, \varpi}^{\phi}=\mathfrak{h}^{\phi} .
$$

From 10.3(b) we have

$$
\mathfrak{h}=\oplus_{N \in \mathbf{Z},(\alpha, n) \in \mathcal{R}_{\underline{N}} ; n=2 N / \eta,\langle\varpi: \alpha\rangle=0}\left(\mathfrak{g}_{\underline{N}}^{\alpha, n}\right) .
$$

Using this and the definitions we have

$$
\begin{aligned}
\{y \in \mathfrak{h} ;[y, h]=0\} & =\oplus_{N \in \mathbf{Z},(\alpha, n) \in \mathcal{R}_{\underline{N}} ; n=2 N / \eta=0,\langle\varpi: \alpha\rangle=0}\left(\mathfrak{g}_{\underline{N}}^{\alpha, n}\right) \\
& =\oplus_{(\alpha, n) \in \mathcal{R}_{\underline{0}} ; n=0,\langle\varpi: \alpha\rangle=0}\left(\mathfrak{g}_{\underline{0}}^{\alpha, n}\right)
\end{aligned}
$$

and (a) follows.

11.4. Recall that $\varpi \in \mathbf{E}^{\prime \prime}$. Let $\mathfrak{p}_{*}={ }^{\epsilon} \mathfrak{p}_{*}^{\varpi}, \mathfrak{u}_{*}={ }^{\epsilon} \mathfrak{u}_{*}$. We can find $\lambda^{\prime} \in Y_{Z}$ such that $\left\langle\lambda^{\prime}: \alpha\right\rangle \neq 0$ for any $i$ and any $(\alpha, n) \in \mathcal{R}_{i}^{*}$. Let $\varpi^{\prime}=\varpi+\frac{1}{b} \lambda^{\prime} \in \mathbf{E}$. Let $\mathfrak{p}_{*}^{\prime}=\epsilon_{\mathfrak{p}_{*}}^{\varpi^{\prime}}, \mathfrak{u}_{*}^{\prime}=\epsilon_{\mathfrak{u}_{*}}^{\varpi^{\prime}}$ and $\mathfrak{m}_{*}^{\prime}=\epsilon \tilde{\mathfrak{l}}_{*}^{\varpi^{\prime}}$. Assume that $b$ is sufficiently large; then $\varpi^{\prime} \in \mathbf{E}^{\prime}$ hence by 10.2 we have $\mathfrak{m}_{*}^{\prime}=\mathfrak{m}_{*}$. The same argument as in 10.6(a) shows that $\mathfrak{p}_{N}^{\prime} \subset \mathfrak{p}_{N}$ for all $N$. Since $b$ is large and $\varpi^{\prime}=\varpi+\frac{1}{b} \lambda^{\prime}$, we see that $\varpi^{\prime}, \varpi$ are very close, so that

$$
\varpi^{\prime} \sim \varpi
$$

For $N \in \mathbf{Z}$ let $\mathfrak{q}_{N}$ be the image of $\mathfrak{p}_{N}^{\prime}$ under the obvious projection $\mathfrak{p}_{N} \rightarrow \mathfrak{h}_{N}$. From 10.5(a),(b), we see that $\mathfrak{q}=\oplus_{N} \mathfrak{q}_{N}$ is a parabolic subalgebra of $\mathfrak{h}$ and $\mathfrak{m}$ is a Levi subalgebra of $\mathfrak{q}$. Moreover, if $u_{N}$ is the image of $\mathfrak{u}_{N}^{\prime}$ under $\mathfrak{p}_{N}$, then $u=\oplus_{N} u_{N}$ is the nilradical of $\mathfrak{q}$.

From 10.3(b) we see that the $\mathbf{Z}$-grading of $\mathfrak{h}$ is $\eta$-rigid and that $e \in \stackrel{\circ}{\mathfrak{h}}_{\eta}$, so that $\stackrel{\circ}{\mathfrak{m}}_{\eta} \subset \stackrel{\circ}{\mathfrak{h}}_{\eta}$. Let $A_{\varpi} \in \mathcal{Q}\left(\mathfrak{h}_{\eta}\right)$ be the simple perverse sheaf on $\mathfrak{h}_{\eta}$ such that the support of $A_{\varpi}$ is $\mathfrak{h}_{\eta}$ and $\left.A_{\varpi}\right|_{\mathfrak{m}_{\eta}}$ is equal up to shift to $\left.\tilde{C}\right|_{\mathfrak{m}_{\eta}}$.

Applying 1.8(b) and the transitivity formula 4.2(a) we deduce

$$
\begin{aligned}
& I_{\varpi^{\prime}}=\widetilde{\operatorname{Ind}}_{\mathfrak{p}_{\eta}^{\prime}}^{\mathfrak{g}_{\delta}}(\tilde{C})=\widetilde{\operatorname{Ind}}_{\mathfrak{p}_{\eta}}^{\mathfrak{g}_{\delta}}\left(\operatorname{ind}_{\mathfrak{q}_{\eta}}^{\mathfrak{h}_{\eta}}(\tilde{C})\right)\left[\operatorname{dim} \mathfrak{u}_{0}^{\prime}+\operatorname{dim} \mathfrak{u}_{\eta}^{\prime}-\operatorname{dim} \mathfrak{u}_{0}-\operatorname{dim} \mathfrak{u}_{\eta}\right] \\
& \cong \oplus_{j} \widetilde{\operatorname{Ind}}_{\mathfrak{p}_{\eta}}^{\mathfrak{g}_{\delta}}\left(A_{\varpi}\right)\left[-2 s_{j}\right]\left[\operatorname{dim} \mathfrak{m}_{\eta}-\operatorname{dim} \mathfrak{h}_{\eta}+\operatorname{dim} \mathfrak{u}_{0}^{\prime}+\operatorname{dim} \mathfrak{u}_{\eta}^{\prime}\right. \\
& \left.-\operatorname{dim} \mathfrak{u}_{0}-\operatorname{dim} \mathfrak{u}_{\eta}\right] \\
& =\oplus_{j} \widetilde{\operatorname{Ind}}_{\mathfrak{p}_{\eta}}^{\mathfrak{g}_{\delta}}\left(A_{\varpi}\right)\left[-2 s_{j}\right][d(\varpi)],
\end{aligned}
$$

where we have used the equality:

$$
\operatorname{dim} \mathfrak{m}_{\eta}-\operatorname{dim} \mathfrak{h}_{\eta}+\operatorname{dim} \mathfrak{u}_{0}^{\prime}+\operatorname{dim} \mathfrak{u}_{\eta}^{\prime}-\operatorname{dim} \mathfrak{u}_{0}-\operatorname{dim} \mathfrak{u}_{\eta}=d(\varpi) .
$$

We now prove (c). Let $u^{\prime}$ be the nilradical of the parabolic subalgebra of $\mathfrak{h}$ that contains $\mathfrak{m}$ and is opposed to $\mathfrak{q}$. We have $u^{\prime}=\oplus_{N} u_{N}^{\prime}$ where $u_{N}^{\prime}=u^{\prime} \cap \mathfrak{h}_{N}$. Now $u_{0}, u_{0}^{\prime}$ are nilradicals of two opposite parabolic subalgebras of $\mathfrak{h}_{0}$ hence $\operatorname{dim} u_{0}=$ $\operatorname{dim} u_{0}^{\prime}$. We have $\operatorname{dim} \mathfrak{h}_{\eta}=\operatorname{dim} u_{\eta}+\operatorname{dim} u_{\eta}^{\prime}+\operatorname{dim} \mathfrak{m}_{\eta}, \operatorname{dim} \mathfrak{u}_{0}^{\prime}-\operatorname{dim} \mathfrak{u}_{0}=\operatorname{dim} u_{0}$, $\operatorname{dim} \mathfrak{u}_{\eta}^{\prime}-\operatorname{dim} \mathfrak{u}_{\eta}=\operatorname{dim} u_{\eta}$. Hence the left-hand side of (c) equals $\operatorname{dim} u_{0}-\operatorname{dim} u_{\eta}^{\prime}=$ $\operatorname{dim} u_{0}^{\prime}-\operatorname{dim} u_{\eta}^{\prime}$. Now $u^{\prime}$ is normalized by $\mathfrak{m}$ hence by the Lie subalgebra $\mathfrak{s}$ of $\mathfrak{m}$ spanned by $e, h, f$. Note that $u_{0}^{\prime}$ (resp. $u_{\eta}^{\prime}$ ) is the 0-(resp. 2-) eigenspace of $\operatorname{ad}(h): u^{\prime} \rightarrow u^{\prime}$. By the representation theory of $\mathfrak{s}$, the $\operatorname{map} \operatorname{ad}(e): u_{0}^{\prime} \rightarrow u_{\eta}^{\prime}$ is surjective and its kernel is exactly the space of $\mathfrak{s}$-invariants in $u^{\prime}$ that is $u^{\prime} \cap \mathfrak{h}^{\phi}$. We see that $\operatorname{dim} u_{0}^{\prime}-\operatorname{dim} u_{\eta}^{\prime}=\operatorname{dim}\left(u^{\prime} \cap \mathfrak{h}^{\phi}\right)$. Now $u^{\prime} \cap \mathfrak{h}^{\phi}$ is the nilradical of a 
parabolic subalgebra of $\mathfrak{h}^{\phi}$ with Levi subgroup $\mathfrak{m} \cap \mathfrak{h}^{\phi}=\mathfrak{z}$ (see 11.1) hence is the nilradical of a Borel subalgebra of $\mathfrak{h}^{\phi}$ (which equals $\mathfrak{g}_{0, \varpi}^{\phi}$ by 11.3(a)). By definition, the dimension of this nilradical is equal to $d(\varpi)$. This proves $(c)$ and hence also (b).

Now (b) implies the following equality in ${ }^{\xi} \mathcal{K}\left(\mathfrak{g}_{\delta}\right)$ :

$$
I_{\varpi}=a_{\varpi}\left(\widetilde{\operatorname{Ind}}_{\mathfrak{p}_{\eta}}^{\mathfrak{g}_{\delta}}\left(A_{\varpi}\right)\right) .
$$

Using this and (a) we see that for any $\mathbf{c} \in \underline{\mathbf{E}}$ and any $\varpi \in \mathbf{E}^{\prime \prime} \cap \mathbf{c}$ we have

$$
a_{\varpi}^{-1} \tilde{\gamma}\left(\tilde{T}_{\mathbf{c}}\right)={ }^{\epsilon} \widetilde{\operatorname{Ind}}_{\mathfrak{p}_{\eta}}^{\mathfrak{g}_{\delta}}\left(A_{\varpi}\right) \in \mathbf{Q}(v) \otimes_{\mathcal{A}} \xi \mathcal{K}\left(\mathfrak{g}_{\delta}\right) .
$$

Since $\widetilde{\operatorname{Ind}}_{\mathfrak{p}_{\eta}}^{\mathfrak{g}_{\delta}}\left(A_{\varpi}\right) \in{ }^{\xi} \mathcal{K}\left(\mathfrak{g}_{\delta}\right)$, we see that:

(e) For any $\mathbf{c} \in \underline{\circ}$ 妾 and any $\varpi \in \mathbf{E}^{\prime \prime} \cap \mathbf{c}$ we have $a_{\varpi}^{-1} \tilde{\gamma}\left(\tilde{T}_{\mathbf{c}}\right) \in{ }^{\xi} \mathcal{K}\left(\mathfrak{g}_{\delta}\right)$.

The following result will be proved in 11.7 .

(f) The $\mathcal{A}$-module ${ }^{\xi} \mathcal{K}\left(\mathfrak{g}_{\delta}\right)$ is generated by the elements $a_{\varpi}^{-1} \tilde{\gamma}\left(\tilde{T}_{\mathbf{c}}\right)$ for various $\mathbf{c} \in \underline{\circ}$ and $\varpi \in \mathbf{E}^{\prime \prime} \cap \mathbf{c}$.

From (f) we deduce:

(g) The isomorphism $\gamma: \mathbf{V} \stackrel{\sim}{\rightarrow} \mathbf{Q}(v) \otimes_{\mathcal{A}} \xi \mathcal{K}\left(\mathfrak{g}_{\delta}\right)$ in Proposition 10.19 (a) restricts to an isomorphism of $\mathcal{A}$-modules $\gamma_{\mathcal{A}}: \mathbf{V}_{\mathcal{A}} \stackrel{\sim}{\rightarrow} \xi \mathcal{K}\left(\mathfrak{g}_{\delta}\right)$.

11.5. Let $\mathfrak{p}_{*}$ be an $\epsilon$-spiral with a splitting $\mathfrak{h}_{*}$ and with nilradical $\mathfrak{u}_{*}$; let $A \in \mathcal{Q}\left(\mathfrak{h}_{\eta}\right)$ be a simple perverse sheaf. We show:

(a) Let $\mathcal{X}$ be the collection of all $B \in \mathfrak{B}$ such that some shift of $B$ is a direct summand of $\widetilde{\operatorname{Ind}_{\mathfrak{p}_{\eta}}}(A)$. Then the map $\mathcal{X} \rightarrow \underline{\mathfrak{T}}_{\eta}, B \mapsto \psi(B)$ is constant.

We can find a parabolic subalgebra $\mathfrak{q}$ of $\mathfrak{h}$ and a Levi subalgebra $\mathfrak{m}^{\prime}$ of $\mathfrak{q}$ such that $\mathfrak{q}=\oplus_{N} \mathfrak{q}_{N}, \mathfrak{m}^{\prime}=\oplus_{N} \mathfrak{m}_{N}^{\prime}$ where $\mathfrak{q}_{N}=\mathfrak{q} \cap \mathfrak{h}_{N}, \mathfrak{m}_{N}^{\prime}=\mathfrak{m}^{\prime} \cap \mathfrak{h}_{N}$ and a cuspidal perverse sheaf $C$ in $\mathcal{Q}\left(\mathfrak{m}_{\eta}\right)$ such that some shift of $A$ is a direct summand of $\operatorname{ind}_{\mathfrak{q}_{\eta}}^{\mathfrak{h}_{\eta}}(C)$. Let $M^{\prime}=e^{\mathfrak{m}^{\prime}}, M_{0}^{\prime}=e^{\mathfrak{m}_{0}^{\prime}}$. Setting $\mathfrak{p}_{N}^{\prime}=\mathfrak{u}_{N}+\mathfrak{q}_{N}$ for any $N \in \mathbf{Z}$, we see from [LY, 2.8(a)] that $\mathfrak{p}_{*}^{\prime}$ is an $\epsilon$-spiral and from [LY, 2.8(b)] that $\mathfrak{m}_{*}^{\prime}$ is a splitting of $\mathfrak{p}_{*}^{\prime}$. We see that $\left(M^{\prime}, M_{0}^{\prime}, \mathfrak{m}^{\prime}, \mathfrak{m}_{*}^{\prime}, C\right) \in \mathfrak{T}_{\eta}$. Let $\xi^{\prime}$ be the element of $\underline{\mathfrak{T}}_{\eta}$ determined by $\left(M^{\prime}, M_{0}^{\prime}, \mathfrak{m}^{\prime}, \mathfrak{m}_{*}^{\prime}, C\right)$. If $B \in \mathcal{X}$, then (by [LY, 4.2]) some shift of $B$ is a direct summand of $\widetilde{\operatorname{Ind}_{\mathfrak{p}_{\eta}^{\prime}}^{\prime}} \mathfrak{g}_{j}(C)$ hence $\psi(B)=\xi^{\prime}$. This proves (a).

We say that $\widetilde{\operatorname{Ind}_{\mathfrak{p}_{\eta}}} \mathfrak{g}_{\delta}(A)$ (as in (a)) has type $\xi^{\prime} \in \underline{\mathfrak{T}}_{\eta}$ if $\psi(B)=\xi^{\prime}$ for any $B \in \mathcal{X}$.

11.6. Recall from $8.4(\mathrm{a})$ that the $\mathcal{A}$-module $\mathcal{K}\left(\mathfrak{g}_{\delta}\right)$ is generated by the classes of $\epsilon$-quasi-monomial objects of $\mathcal{Q}\left(\mathfrak{g}_{\delta}\right)$. Using this and $11.5(\mathrm{a})$, we deduce that in the direct sum decomposition $\mathcal{K}\left(\mathfrak{g}_{\delta}\right)=\oplus_{\xi \in \underline{\mathfrak{T}}_{\eta}}{ }^{\xi} \mathcal{K}\left(\mathfrak{g}_{\delta}\right)$ (see [LY, 6.7]), any summand $\xi \mathcal{K}\left(\mathfrak{g}_{\delta}\right)$ is generated as an $\mathcal{A}$-module by the classes of $\eta$-quasi-monomial objects in $\mathcal{Q}\left(\mathfrak{g}_{\delta}\right)$ of type $\xi$.

11.7. We prove 11.4(f). (Thus we are again in the setup of 11.1.) Using 11.6, we see that it is enough to show that if $A^{\prime}$ is an $\eta$-quasi-monomial object in $\mathcal{Q}\left(\mathfrak{g}_{\delta}\right)$ of type $\xi$, then the class of $A^{\prime}$ in $\mathcal{K}\left(\mathfrak{g}_{\delta}\right)$ is of the form $a_{\varpi}^{-1} \tilde{\gamma}\left(\tilde{T}_{\mathbf{c}}\right)$ for some $\mathbf{c} \in \stackrel{\circ}{\mathbf{E}}$ and $\varpi \in \mathbf{E}^{\prime \prime} \cap \mathbf{c}$. We can find:

(a) $\mathfrak{p}_{*}, \mathfrak{h}_{*}, A, \mathfrak{q}_{*}, \mathfrak{p}_{*}^{\prime},\left(M^{\prime}, M_{0}^{\prime}, \mathfrak{m}^{\prime}, \mathfrak{m}_{*}^{\prime}, C\right) \in \mathfrak{T}_{\eta}$ (representing $\xi$ ) as in 11.5 such that $A^{\prime}=\widetilde{\operatorname{Ind}_{\mathfrak{p}_{\eta}}}(A)$; moreover, we can assume that the $\mathbf{Z}$-grading of $\mathfrak{h}=\oplus_{N} \mathfrak{h}_{N}$ is 
$\eta$-rigid and $\stackrel{\circ}{\mathfrak{m}_{\eta}^{\prime}} \subset \mathfrak{h}_{\eta}^{\circ}$. Replacing the data (a) by a $G_{\underline{0}^{-}}$-conjugate we can assume in addition that $\left(M^{\prime}, M_{0}^{\prime}, \mathfrak{m}^{\prime}, \mathfrak{m}_{*}^{\prime}, C\right)$ is equal to $\left(M, M_{0}, \mathfrak{m}, \mathfrak{m}_{*}, \tilde{C}\right)$ in 10.1 .

Let $H=e^{\mathfrak{h}}$. Since $\mathfrak{h}_{*}$ is $\eta$-rigid, there exists $\iota^{\prime} \in Y_{H}$ such that:

(i) ${ }_{k}^{\iota^{\prime}}=\mathfrak{h}_{k \eta / 2}$ if $k \in \mathbf{Z}, k \eta / 2, \stackrel{\iota^{\prime}}{\stackrel{k}{\prime}=0}$ if $k \in \mathbf{Z}, k \eta / 2 \notin \mathbf{Z}$ and

(ii) $\iota^{\prime}=\iota_{\phi^{\prime}}$ for some $\phi^{\prime}=\left(e^{\prime}, h^{\prime}, f^{\prime}\right) \in J^{H}$ such that $e^{\prime} \in \stackrel{\circ}{h}_{\eta}, h^{\prime} \in \mathfrak{h}_{0}, f^{\prime} \in \mathfrak{h}_{-\eta}$.

Since $\stackrel{\circ}{\mathfrak{m}}_{\eta} \subset \stackrel{\circ}{\mathfrak{h}}_{\eta}$ and $e \in \stackrel{\circ}{\mathfrak{m}}_{\eta}$, we see that $e, e^{\prime}$ are in the same $M_{0}$-orbit. Hence we can find $g \in M_{0}$ such that $\operatorname{Ad}(g)$ conjugates $e^{\prime}, f^{\prime}, h^{\prime}, \iota^{\prime}$ to $e, f, h, \iota$. Applying $\operatorname{Ad}(g)$ (which preserves $\mathfrak{h}_{k}$ ) to (i) and (ii) we see that we can assume that $\iota^{\prime}=\iota, \phi^{\prime}=\phi$.

Recall from 10.3 that $\tilde{\mathfrak{l}}_{N}^{\phi}={ }_{2 N / \eta}^{\iota} \mathfrak{g}_{N}$ for $N \in \mathbf{Z}$ such that $2 N / \eta \in \mathbf{Z}$. Using (i) with $\iota^{\prime}=\iota$ we see that $\mathfrak{h}_{N} \subset \tilde{\mathfrak{l}}_{N}^{\phi}$ for any $N \in \mathbf{Z}$ such that $2 N / \eta \in \mathbf{Z}$. Using 10.4(c),(d) we see that for some $\varpi \in \mathbf{E}^{\prime \prime}$ we have $\mathfrak{p}_{*}={ }^{\epsilon} \mathfrak{p}_{*}^{\varpi}, \mathfrak{h}_{*}={ }^{\epsilon} \tilde{\mathfrak{l}}_{*}^{\varpi}$. Using now $11.4(\mathrm{~d})$, we see that $A^{\prime}=a_{\varpi}^{-1} \tilde{\gamma}\left(\tilde{T}_{\mathbf{c}}\right)$, where $\mathbf{c} \in \underline{\mathbf{E}}$ contains $\varpi$. This completes the proof of $11.4(\mathrm{f})$, hence that of $11.4(\mathrm{~g})$.

11.8. We can now prove $11.2(\mathrm{~b})$. Using $11.4(\mathrm{~g}), 11.2(\mathrm{~b})$ is reduced to the following obvious statement: ${ }^{\xi} \mathcal{K}\left(\mathfrak{g}_{\delta}\right)$ is a free $\mathcal{A}$-module.

11.9. We define a $\mathbf{Q}$-linear map

$$
{ }^{-}: \mathbf{Q}(v) \otimes_{\mathcal{A}} \mathcal{K}\left(\mathfrak{g}_{\delta}\right) \rightarrow \mathbf{Q}(v) \otimes_{\mathcal{A}} \mathcal{K}\left(\mathfrak{g}_{\delta}\right)
$$

by $\overline{f B}=\bar{f} B$ for any $f \in \mathbf{Q}(v)$ and any $B \in \mathfrak{B}$ (see 10.13); here $\bar{f}$ is as in 0.12 . This restricts to a $\mathbf{Q}$-linear map

$$
{ }^{-}: \mathbf{Q}(v) \otimes_{\mathcal{A}}{ }^{\xi} \mathcal{K}\left(\mathfrak{g}_{\delta}\right) \rightarrow \mathbf{Q}(v) \otimes_{\mathcal{A}}{ }^{\xi} \mathcal{K}\left(\mathfrak{g}_{\delta}\right)
$$

and to a $\mathbf{Z}$-linear map ${ }^{\xi} \mathcal{K}\left(\mathfrak{g}_{\delta}\right) \rightarrow{ }^{\xi} \mathcal{K}\left(\mathfrak{g}_{\delta}\right)$. We show:

(a) $\overline{I_{\varpi}}=I_{\varpi}$ for any $\varpi \in \mathbf{E}^{\prime}$.

In ${ }^{\xi} \mathcal{Q}\left(\mathfrak{g}_{\delta}\right)$ we have $I_{\varpi}=\sum_{B \in \xi \mathfrak{B}} f_{B} B$ where $f_{B} \in \mathcal{A}$. It is enough to prove that $\bar{f}_{B}=f_{B}$ for all $B$.

We set $\mathfrak{p}_{*}={ }^{\epsilon} \mathfrak{p}_{*}^{\varpi}$. Let $\sigma$ be an automorphism of order 2 of $\overline{\mathbf{Q}}_{l}$ such that $\sigma(z)=$ $z^{-1}$ for any root of 1 in $\overline{\mathbf{Q}}_{l}$. Applying $\sigma$ to $K \in \mathcal{Q}\left(\mathfrak{m}_{\eta}\right)$ (resp. $K \in \mathcal{Q}\left(\mathfrak{g}_{\delta}\right)$ ) we obtain $K^{\sigma} \in \mathcal{Q}\left(\mathfrak{m}_{\eta}\right)$ (resp. $\left.K^{\sigma} \in \mathcal{Q}\left(\mathfrak{g}_{\delta}\right)\right)$. Note that $K \mapsto K^{\sigma}$ commutes with shifts; moreover, we have

$$
\left(\widetilde{\operatorname{Ind}}_{\mathfrak{p}_{\eta}}^{\mathfrak{g}_{\delta}}(\tilde{C})\right)^{\sigma}=\widetilde{\operatorname{Ind}}_{\mathfrak{p}_{\eta}}^{\mathfrak{g}_{\delta}}\left(\tilde{C}^{\sigma}\right) .
$$

Moreover, if $K$ is a simple perverse sheaf in $\mathcal{Q}\left(\mathfrak{m}_{\eta}\right)$ or in $\mathcal{Q}\left(\mathfrak{g}_{\delta}\right)$, we have

$$
K^{\sigma} \cong D(K),
$$

since $K$ restricted to an open dense subset of it support is a local system with finite monodromy. By (b),(c) and [LY, 4.1(d)] we have

$$
D\left(\widetilde{\operatorname{Ind}}_{\mathfrak{p}_{\eta}}^{\mathfrak{g}_{\delta}}(\tilde{C})\right)={\widetilde{\operatorname{Ind}_{\mathfrak{p}_{\eta}}}}_{\mathfrak{g}_{\delta}}^{\mathfrak{g}}(D(\tilde{C}))=\widetilde{ }^{\epsilon} \widetilde{\operatorname{Ind}}_{\mathfrak{p}_{\eta}}^{\mathfrak{g}_{\delta}}\left(\tilde{C}^{\sigma}\right)=\left(\widetilde{\operatorname{Ind}_{\mathfrak{p}_{\eta}}}(\tilde{C})\right)^{\sigma}
$$

hence

$$
\left(D\left(I_{\varpi}\right)\right)^{\sigma}=I_{\varpi}
$$

and $\sum_{B} \bar{f}_{B} D\left(B^{\sigma}\right)=\sum_{B} f_{B} B$. Using this and (c) for $K=B$, we see that $\bar{f}_{B}=f_{B}$ for all $B$; this proves (a). 
11.10. We show:

$(\text { a) })^{-}: \mathbf{V}^{\prime} \rightarrow \mathbf{V}^{\prime}$ (see 10.21) restricts to an involution $\mathbf{V}_{\mathcal{A}}^{\prime} \rightarrow \mathbf{V}_{\mathcal{A}}^{\prime}$ and $d^{-}: \mathbf{V} \rightarrow \mathbf{V}$ (see 10.21) restricts to an involution $\mathbf{V}_{\mathcal{A}} \rightarrow \mathbf{V}_{\mathcal{A}}$ (these restrictions are denoted again by .

It is enough to note that for any $\varpi \in \mathbf{E}^{\prime \prime}$ we have $\overline{a_{\varpi}}=a_{\varpi}$.

We show:

(b) $\tilde{\gamma}: \mathbf{V}^{\prime} \rightarrow \mathbf{Q}(v) \otimes_{\mathcal{A}}{ }^{\xi} \mathcal{K}\left(\mathfrak{g}_{\delta}\right)$ is compatible with the maps ${ }^{-}$in the two sides.

This follows from $11.9(\mathrm{a})$.

We have the following result.

\section{Proposition 11.11.}

(a) Let $\mathbf{B}^{\prime}$ be the set of all $b \in \mathbf{V}_{\mathcal{A}}$ such that $\bar{b}=b$ and $(b: b) \in 1+v \mathbf{Z}[[v]]$. Then $\mathbf{B}^{\prime}$ is a signed basis of the $\mathcal{A}$-module $\mathbf{V}_{\mathcal{A}}$ (that is, the union of a basis with $(-1)$ times that basis).

(b) For $b \in \mathbf{B}^{\prime}$ we have $(b: b) \in 1+v \mathbf{N}[[v]]$.

(c) There is a unique $\mathcal{A}$-basis $\mathbf{B}$ of $\mathbf{V}_{\mathcal{A}}$ such that $\mathbf{B} \subset \mathbf{B}^{\prime}$ and for any $\mathbf{c} \in \underline{\mathbf{E}}$ and any $\varpi \in \mathbf{E}^{\prime \prime} \cap \mathbf{c}$, the image of $a_{\varpi}^{-1} \tilde{T}_{\mathbf{c}}$ in $\mathbf{V}_{\mathcal{A}}$ is an $\mathbf{N}\left[v, v^{-1}\right]$-linear combination of elements in $\mathbf{B}$.

It is enough to prove the analogous statements where $\mathbf{V}_{\mathcal{A}}$ is identified via $\gamma$ with ${ }^{\xi} \mathcal{K}\left(\mathfrak{g}_{\delta}\right)$ with (:) as in $4.4(\mathrm{c})$ and with ${ }^{-}$as in 11.9 (we use $10.19(\mathrm{~b}), 11.10(\mathrm{~b})$ ). Let $\xi_{\mathfrak{B}}=\left\{B_{1}, B_{2}, \ldots, B_{r}\right\}$ (see 10.13). From 0.12 we have $\left(B_{j}: B_{j^{\prime}}\right) \in \delta_{j, j^{\prime}}+h_{j, j^{\prime}}$, where $h_{j, j^{\prime}} \in v \mathbf{N}[[v]]$ for all $j, j^{\prime}$ in $[1, r]$. From the definition (see 11.9) we have $\overline{B_{j}}=B_{j}$ for $j=1, \ldots, r$. Now let $b \in{ }^{\xi} \mathcal{K}\left(\mathfrak{g}_{\delta}\right)$ be such that $\bar{b}=b$ and $(b: b) \in$ $1+v \mathbf{Z}[[v]]$. To prove (a), it is enough to show that $b= \pm B_{j}$ for some $j$. We can write $b=\sum_{j=1}^{r} f_{j} B_{j}$, where $f_{j} \in \mathcal{A}$ satisfy $\bar{f}_{j}=f_{j}$ and $\sum_{j, j^{\prime} \in[1, r]} \bar{f}_{j} f_{j^{\prime}}\left(\delta_{j, j^{\prime}}+h_{j, j^{\prime}}\right) \in$ $1+v \mathbf{Z}[[v]]$ hence $\sum_{j, j^{\prime} \in[1, r]} f_{j} f_{j^{\prime}}\left(\delta_{j, j^{\prime}}+h_{j, j^{\prime}}\right) \in 1+v \mathbf{Z}[[v]]$. We can find $c \in \mathbf{Z}$ such that $f_{j}=f_{j, c} v^{c} \bmod v^{c+1} \mathbf{Z}[v]$ where $f_{j, c} \in \mathbf{Z}$ for all $j$ and $f_{j, c} \neq 0$ for some $j$. We have $\sum_{j \in[1, r]} f_{j, c}^{2} v^{2 c}+v^{2 c+1} f^{\prime}=1+v f^{\prime \prime}$ where $f^{\prime}, f^{\prime \prime} \in \mathbf{Z}[v]$. Moreover, $\sum_{j \in[1, r]} f_{j, c}^{2}>0$. It follows that $c=0$ and $\sum_{j \in[1, r]} f_{j, 0}^{2}=1$ so that there exists $j_{0} \in[1, r]$ such that $f_{j_{0}, 0}= \pm 1$ and $f_{j, 0}=0$ for $j \neq j_{0}$. We have $f_{j}= \pm \delta_{j, j_{0}}$ $\bmod v \mathbf{Z}[v]$ for all $j$. Since $\bar{f}_{j}=f_{j}$ we deduce that $f_{j}= \pm \delta_{j, j_{0}}$ for all $j$. Thus $b= \pm B_{j_{0}}$. This completes the proof of (a). At the same time we have proved (b). Clearly, $\left\{B_{1}, B_{2}, \ldots, B_{r}\right\}$ has the positivity property in (c) (with $\mathbf{V}_{\mathcal{A}}$ identified with ${ }^{\xi} \mathcal{K}\left(\mathfrak{g}_{\delta}\right)$ and with $a_{\varpi}^{-1} \tilde{T}_{\mathbf{c}}$ identified with $\left.\gamma\left(a_{\varpi}^{-1} \tilde{T}_{\mathbf{c}}\right)\right)$. Since any $B_{j}$ appears with $>0$ coefficient in some $\gamma\left(a_{\varpi}^{-1} \tilde{T}_{\mathbf{c}}\right)$, we see that $\left\{B_{1}, B_{2}, \ldots, B_{r}\right\}$ is the only basis contained in $\left\{ \pm B_{1}, \pm B_{2}, \ldots, \pm B_{r}\right\}$ with the positivity property in (c). This completes the proof of the proposition.

11.12. From the proof of 11.11 we see that $\gamma: \mathbf{V} \stackrel{\sim}{\rightarrow} \mathbf{Q}(v) \otimes_{\mathcal{A}} \xi \mathcal{K}\left(\mathfrak{g}_{\delta}\right)$ (see Proposition $10.19(\mathrm{a}))$ restricts to a bijection

$$
\mathbf{B} \stackrel{\sim}{\rightarrow} \xi_{\mathfrak{B}} .
$$

For any $G_{\underline{0}}$-orbit $\mathcal{O}$ in $\mathfrak{g}_{\delta}^{\text {nil }}$ let $\mathbf{B}_{\mathcal{O}}$ be the set of all $b \in \mathbf{B}$ such that $\gamma(b) \in \mathfrak{B}_{\mathcal{O}}$ (see 10.13). We have a partition $\mathbf{B}=\sqcup_{\mathcal{O}} \mathbf{B}_{\mathcal{O}}$ where $\mathcal{O}$ runs over the $G_{\underline{0}^{-}}$orbits in $\mathfrak{g}_{\delta}^{\text {nil }}$. 
11.13. We consider the setup of 10.22. We show:

(a) The $\mathcal{A}$-submodule $\mathbf{V}_{\mathcal{A}}$ of $\mathbf{V}$ defined in 11.2 in terms of $\dot{\xi}$ is the same as that defined in terms of $\dot{\xi}_{1}$.

It is not clear how to prove this using the definition in 11.2 since $\mathbf{E}^{\prime \prime}$ defined in terms of $\dot{\xi}$ (see 10.3) is not necessarily the same as that defined in terms of $\dot{\xi}_{1}$. Instead we will argue indirectly. Using $11.4(\mathrm{~g})$ it is enough to show:

(b) The isomorphism $\gamma: \mathbf{V} \stackrel{\sim}{\rightarrow} \mathbf{Q}(v) \otimes_{\mathcal{A}} \xi \mathcal{K}\left(\mathfrak{g}_{\delta}\right)$ defined in 10.19 in terms of $\dot{\xi}$ is equal to the analogous isomorphism defined in terms of $\dot{\xi}_{1}$.

Thus it is enough to show that if $\varpi \in \mathbf{E}^{\prime} \cap \mathbf{E}_{1}^{\prime}$, then $\tilde{I}_{\varpi}$ defined in 10.2 in terms of $\dot{\xi}$ is the same as that defined in terms of $\dot{\xi}_{1}$. Using the definitions we see that it is enough to show that

or that

$$
\begin{aligned}
& \dot{\eta}_{\mathfrak{p}_{\eta}}(|\eta| / 2)(\varpi+\iota)=\dot{\eta}_{1} \mathfrak{p}_{\eta_{1}}^{\left(\left|\eta_{1}\right| / 2\right)(\varpi+\iota)} \\
& \dot{\eta}_{\mathfrak{p}_{0}}^{(|\eta| / 2)(\varpi+\iota)}=\dot{\eta}_{1} \mathfrak{p}_{0}^{\left(\left|\eta_{1}\right| / 2\right)(\varpi+\iota)} .
\end{aligned}
$$

$$
\begin{aligned}
& \left.\oplus_{\kappa \in \mathbf{Q} ; \kappa \geq|\eta|}\left({ }_{\kappa}^{(|\eta| / 2)(\varpi+\iota)} \mathfrak{g}_{\delta}\right)=\oplus_{\kappa \in \mathbf{Q} ; \kappa \geq\left|\eta_{1}\right|\left({ }_{\kappa}\left(\left|\eta_{1}\right| / 2\right)(\varpi+\iota)\right.} \mathfrak{g}_{\delta}\right), \\
& \oplus_{\kappa \in \mathbf{Q} ; \kappa \geq 0}\left({ }_{\kappa}^{(|\eta| / 2)(\varpi+\iota)} \mathfrak{g}_{\delta}\right)=\oplus_{\kappa \in \mathbf{Q} ; \kappa \geq 0}\left({ }_{\kappa}^{\left(\left|\eta_{1}\right| / 2\right)(\varpi+\iota)} \mathfrak{g}_{\delta}\right),
\end{aligned}
$$

or that

$$
\begin{aligned}
& \oplus_{\kappa \in \mathbf{Q} ; \kappa /|\eta| \geq 1}\left(\begin{array}{l}
(1 / 2)(\varpi+\iota) \\
\kappa /|\eta|
\end{array} \mathfrak{g}_{\delta}\right)=\oplus_{\kappa \in \mathbf{Q} ; \kappa /\left|\eta_{1}\right| \geq 1}\left(\begin{array}{l}
\left(\begin{array}{l}
1 / 2)(\varpi+\iota) \\
\kappa /\left|\eta_{1}\right|
\end{array}\right) \\
\mathfrak{g}_{\delta}
\end{array}\right), \\
& \oplus_{\kappa \in \mathbf{Q} ; \kappa /|\eta| \geq 0}\left(\begin{array}{l}
(1 / 2)(\varpi+\iota) \\
\kappa /|\eta|
\end{array} \mathfrak{g}_{\delta}\right)=\oplus_{\kappa \in \mathbf{Q} ; \kappa /\left|\eta_{1}\right| \geq 0}\left(\begin{array}{l}
(1 / 2)(\varpi+\iota) \\
\kappa /\left|\eta_{1}\right|
\end{array} \mathfrak{g}_{\delta}\right),
\end{aligned}
$$

or, setting $\kappa^{\prime}=\kappa /|\eta|, \kappa^{\prime \prime}=\kappa /\left|\eta_{1}\right|$, that

$$
\begin{aligned}
& \bigoplus_{\kappa^{\prime} \in \mathbf{Q} ; \kappa^{\prime} \geq 1}\left(\begin{array}{l}
(1 / 2)(\varpi+\iota) \\
\kappa^{\prime}
\end{array} \mathfrak{g}_{\delta}\right)=\oplus_{\kappa^{\prime \prime} \in \mathbf{Q} ; \kappa^{\prime \prime} \geq 1}\left(\begin{array}{l}
(1 / 2)(\varpi+\iota) \\
\kappa^{\prime \prime}
\end{array} \mathfrak{g}_{\delta}\right), \\
& \left.\bigoplus_{\kappa^{\prime} \in \mathbf{Q} ; \kappa^{\prime} \geq 0}\left(\begin{array}{l}
(1 / 2)(\varpi+\iota) \\
\kappa^{\prime}
\end{array} \mathfrak{g}_{\delta}\right)=\bigoplus_{\kappa^{\prime \prime} \in \mathbf{Q} ; \kappa^{\prime \prime} \geq 0}\left(\begin{array}{l}
(1 / 2)(\varpi+\iota) \\
\kappa^{\prime \prime}
\end{array}\right), \mathfrak{g}_{\delta}\right),
\end{aligned}
$$

which are obvious. This proves (a).

Using (b) and 11.12(b) we see that the basis $\mathbf{B}$ of $\mathbf{V}_{\mathcal{A}}$ defined in 11.11 in terms of $\dot{\xi}$ is the same as that defined in terms of $\dot{\xi}_{1}$.

\section{Purity PRoperties}

In this section we show that for any irreducible local system $\mathcal{L}$ on a $G_{\underline{0}}$-orbit in $\mathfrak{g}_{\delta}^{\text {nil }}$ the cohomology sheaves of $\mathcal{L}^{\sharp} \in \mathcal{D}\left(\mathfrak{g}_{\delta}\right)$ satisfy a strong purity property. This generalizes the analogous result in the $\mathbf{Z}$-graded case in [L4].

12.1. In this section we assume that $p>0$ and that $\mathbf{k}$ is an algebraic closure of a finite field $\mathbf{F}_{q}$ with $q$ elements (here $q$ is a power of $p$ ). Replacing $q$ by larger powers of $p$ if necessary, we can assume that $m$ divides $q-1$ and that we can find an $\mathbf{F}_{q^{-}}$ rational structure on $G$ with Frobenius map $F: G \rightarrow G$ such that $\vartheta: G \rightarrow G$ (see $0.5)$ commutes with $F: G \rightarrow G$. Then $G_{\underline{0}}$ is defined over $\mathbf{F}_{q}$ and $\mathfrak{g}$ inherits from $G$ an $\mathbf{F}_{q}$-rational structure with Frobenius map $F: \mathfrak{g} \rightarrow \mathfrak{g}$ satisfying $F\left(\mathfrak{g}_{i}\right)=\mathfrak{g}_{i}$ for all $i$. Again by replacing $q$ by larger powers of $p$ if necessary, we may assume that all $G_{\underline{0}}$-orbits in $\mathfrak{g}_{\delta}^{\text {nil }}$ are defined over $\mathbf{F}_{q}$ and that for any irreducible $G_{\underline{0}}$-equivariant local system $\mathcal{L}$ on a $G_{\underline{0}^{-}}$orbit $\mathcal{O}$ in $\mathfrak{g}_{\delta}^{\text {nil }}$ we have $F^{*} \mathcal{L} \cong \mathcal{L}$.

We now fix a $G_{\underline{0}}$-orbit $\mathcal{O}$ in $\mathfrak{g}_{\delta}^{\text {nil }}$ with closure $\overline{\mathcal{O}}$ and an irreducible $G_{\underline{0}}$-equivariant local system $\mathcal{L}$ on $\mathcal{O}$. We fix an isomorphism $\tilde{F}: F^{*} \mathcal{L} \rightarrow \mathcal{L}$ which induces the identity map on the stalk of $\mathcal{L}$ at some point of $\mathcal{O}^{F}$. Then $\tilde{F}$ induces an isomorphism (denoted again by $\tilde{F}$ ) $F^{*} \mathcal{L}^{\sharp} \rightarrow \mathcal{L}^{\sharp}$. Given a finite-dimensional $\overline{\mathbf{Q}}_{l}$-vector space $V$ 
with an endomorphism $\tilde{F}: V \rightarrow V$, we say that $\tilde{F}: V \rightarrow V$ is $a$-pure (for an integer a) if the eigenvalues of $\tilde{F}$ are algebraic numbers all of whose complex conjugates have absolute value $q^{a / 2}$. Sometimes we will just say that $V$ is $a$-pure (where this is understood to refer to $\tilde{F}$ ).

12.2. We show:

(a) For any $x \in \overline{\mathcal{O}}^{F}$ and any $a \in \mathbf{Z}$, the induced linear map $\tilde{F}: \mathcal{H}_{x}^{a}\left(\mathcal{L}^{\sharp}\right) \rightarrow \mathcal{H}_{x}^{a}\left(\mathcal{L}^{\sharp}\right)$ is a-pure.

Using [LY, 2.3(b)], we can find $\phi=(e, h, f) \in J_{\delta}(x)$ such that $h, f$ are $\mathbf{F}_{q^{-}}$ rational. Recall that $x=e$. Let $\iota=\iota_{\phi} \in Y_{G}$ be as in 1.1. Let $\mathfrak{z}(f)$ be the centralizer of $f$ in $\mathfrak{g}$ and let $\Sigma=e+\mathfrak{z}(f) \subset \mathfrak{g}$. According to Slodowy:

(b) The affine space $\Sigma$ is a transversal slice at $x$ to the $G$-orbit of $e$ in $\mathfrak{g}$ and the $\mathbf{k}^{*}$-action $t \mapsto t^{-2} \operatorname{Ad}(\iota(t))$ on $\mathfrak{g}$ keeps e fixed, leaves $\Sigma$ stable and defines a contraction of $\Sigma$ to $x$.

Let $\tilde{\Sigma}=\Sigma \cap \mathfrak{g}_{\delta}=e+\left(\mathfrak{z}(f) \cap \mathfrak{g}_{\delta}\right)$. Then:

(c) $\tilde{\Sigma}$ is a transversal slice to the $G_{0}$-orbit of $e$ in $\mathfrak{g}_{\delta}$; the $\mathbf{k}^{*}$-action in (b) leaves stable $\tilde{\Sigma}$ and is a contraction of $\tilde{\Sigma}$ to $e$.

(We have a direct sum decomposition $\mathfrak{g}_{\delta}=\left(\mathfrak{z}(f) \cap \mathfrak{g}_{\delta}\right) \oplus\left[e, \mathfrak{g}_{0}\right]$ obtained by taking the $\zeta^{\delta}$-eigenspace of $\theta: \mathfrak{g} \rightarrow \mathfrak{g}$ in the two sides of the direct sum decomposition $\mathfrak{g}=\mathfrak{z}(f) \oplus[e, \mathfrak{g}] ;$ note that both $\mathfrak{z}(f)$ and $[e, \mathfrak{g}]$ are $\theta$-stable. $)$

Let $\mathcal{L}^{\prime}$ be the restriction of $\mathcal{L}$ to $\mathcal{O} \cap \tilde{\Sigma}$ (a smooth irreducible subvariety of $\tilde{\Sigma}$ ). Note that $\mathcal{O} \cap \tilde{\Sigma}, \overline{\mathcal{O}} \cap \tilde{\Sigma}$ are stable under the $\mathbf{k}^{*}$-action in (c) and $\mathcal{L}^{\prime}$ is equivariant for that action. By $(\mathrm{c})$, we have $\mathcal{H}_{x}\left(\mathcal{L}^{\sharp}\right)=\mathcal{H}_{x}\left(\mathcal{L}^{\prime \sharp}\right)$. It remains to show that $\mathcal{H}_{x}\left(\mathcal{L}^{\prime \sharp}\right)$ is $a$-pure. This can be reduced to Deligne's hard Lefschetz theorem by an argument in Lemma 4.5(b) in KL2 applied to $\overline{\mathcal{O}} \cap \tilde{\Sigma} \subset \tilde{\Sigma}$ with the contraction in (c) and to $\mathcal{L}^{\prime}$ instead of $\overline{\mathbf{Q}}_{l}$. Note that in [KL2, 4.5(b)] an inductive purity assumption was made which is in fact unnecessary, by Gabber purity theorem. This completes the proof of (a).

Erratum to [L4]. On page 209, line -5 , replace $t^{-n} \operatorname{Ad}\left(\iota^{\prime}(t)\right)$ by $t^{-2} \operatorname{Ad}\left(\iota^{\prime}(t)\right)$.

\section{AN INNER PRODUCT}

This section is an adaptation of [L4, §19] from the $\mathbf{Z}$-graded case to the $\mathbf{Z} / m$ graded case; we express the matrix whose entries are the values of the (:)-pairing at two elements of $\mathfrak{B}$ as a product of three matrices. As an application we show (see $13.8(\mathrm{a}))$ that if $(\mathcal{O}, \mathcal{L}),\left(\mathcal{O}^{\prime}, \mathcal{L}^{\prime}\right)$ in $\mathcal{I}\left(\mathfrak{g}_{\delta}\right)$ are such that some cohomology sheaf of $\left.\mathcal{L}^{\sharp}\right|_{\mathcal{O}^{\prime}}$ contains $\mathcal{L}^{\prime}$, then $(\mathcal{O}, \mathcal{L}),\left(\mathcal{O}^{\prime}, \mathcal{L}^{\prime}\right)$ are in the same block. Another application (to odd vanishing) is given in Section 14.

13.1. We fix $(\mathcal{O}, \mathcal{L}),\left(\mathcal{O}^{\prime}, \mathcal{L}^{\prime}\right)$ in $\mathcal{I}\left(\mathfrak{g}_{\delta}\right)$ and we form $A=\mathcal{L}^{\sharp}, A^{\prime}=\mathcal{L}^{\prime \sharp}$ in $\mathcal{D}\left(\mathfrak{g}_{\delta}\right)$. We want to compute $d_{j}\left(\mathfrak{g}_{\delta} ; A, A^{\prime}\right)$ (see [LY, 0.12]) for a fixed $j \in \mathbf{Z}$. We can arrange the $G_{\underline{0}}$-orbits in $\mathfrak{g}_{\delta}^{\text {nil }}$ in an order $\mathcal{O}_{0}, \mathcal{O}_{1}, \ldots, \mathcal{O}_{\beta}$ such that $\mathcal{O}_{\leq s}=\mathcal{O}_{0} \cup \mathcal{O}_{1} \cup \cdots \cup \mathcal{O}_{s}$ is closed in $\mathfrak{g}_{\delta}$ for $s=0,1, \ldots, \beta$. We choose a smooth irreducible variety $\Gamma$ with a free action of $G_{\underline{0}}$ such that $H^{r}\left(\Gamma, \overline{\mathbf{Q}}_{l}\right)=0$ for $r=1,2, \ldots, \mathbf{m}$ where $\mathbf{m}$ is a large integer (compared to $j$ ). We assume that $\mathbf{D}:=\operatorname{dim} \Gamma$ is large (compared to $j$ ). We have $H_{c}^{2 \mathbf{D}-i}\left(\Gamma, \overline{\mathbf{Q}}_{l}\right)=0$ for $i=1,2, \ldots, \mathbf{m}$. We form $X=G_{0} \backslash\left(\Gamma \times \mathfrak{g}_{\delta}\right)$. Let $\tilde{\mathcal{L}}, \tilde{\mathcal{L}}^{\prime}$ be the local systems on the smooth subvarieties $G_{\underline{0}} \backslash(\Gamma \times \mathcal{O}), G_{\underline{0}} \backslash\left(\Gamma \times \mathcal{O}^{\prime}\right)$ of $X$ defined by $\mathcal{L}, \mathcal{L}^{\prime}$ and let $\tilde{A}=\tilde{\mathcal{L}}^{\sharp}, \tilde{A}^{\prime}=\tilde{\mathcal{L}}^{\prime \sharp}$ be the corresponding intersection cohomology complexes in $\mathcal{D}(X)$. Then $\tilde{A} \otimes \tilde{A}^{\prime} \in \mathcal{D}(X)$ is well defined; its restriction to various 
subvarieties of $X$ will be denoted by the same symbol. For $s=0,1, \ldots, \beta$ we form $X_{s}=G_{\underline{0}} \backslash\left(\Gamma \times \mathcal{O}_{s}\right), X_{\leq s}=G_{\underline{0}} \backslash\left(\Gamma \times \mathcal{O}_{\leq s}\right)$. We set $X_{\leq-1}=\emptyset$. The partition of $X_{\leq s}$ into $X_{\leq s-1}$ and $X_{s}$ (for $\left.s=\overline{0}, 1, \ldots, \beta\right)$ gives rise to a long exact sequence

$$
\begin{aligned}
& \ldots \stackrel{\xi_{a}}{\longrightarrow} H_{c}^{a}\left(X_{s}, \tilde{A} \otimes \tilde{A}^{\prime}\right) \rightarrow H_{c}^{a}\left(X_{\leq s}, \tilde{A} \otimes \tilde{A}^{\prime}\right) \\
& \rightarrow H_{c}^{a}\left(X_{\leq s-1}, \tilde{A} \otimes \tilde{A}^{\prime}\right) \stackrel{\xi_{a+1}}{\longrightarrow} H_{c}^{a+1}\left(X_{s}, \tilde{A} \otimes \tilde{A}^{\prime}\right) \rightarrow \ldots
\end{aligned}
$$

13.2. In the following proposition we encounter two kinds of integers; some like $j$, $m, \operatorname{dim} G_{0}, \operatorname{dim} \mathfrak{g}_{\delta}, \beta$ are regarded as "small" (they belong to a fixed finite set of integers), others like $2 \mathbf{D}$ and $\mathbf{m}$ are regarded as very large (we are free to choose them so). We will also encounter integers $a$ such that $2 \mathbf{D}-a$ is a "small" integer (we then write $a \sim 2 \mathbf{D}$ ).

\section{Proposition 13.3.}

(a) Assume that $\mathbf{k}$ is as in 12.1. Then $H_{c}^{a}\left(X_{s}, \tilde{A} \otimes \tilde{A}^{\prime}\right)$ is a-pure (see 12.1) for $s=0,1, \ldots, \beta$ and $a \sim 2 \mathbf{D}$.

(b) We choose $x_{s} \in \mathcal{O}_{s}$. If $a \sim 2 \mathbf{D}$, then

$$
\begin{aligned}
& \operatorname{dim} H_{c}^{a}\left(X_{s}, \tilde{A} \otimes \tilde{A}^{\prime}\right) \\
& =\sum_{r+r_{1}+r_{2}=a} \operatorname{dim}\left(H_{c}^{r}\left(G_{\underline{0}}\left(x_{s}\right)^{0} \backslash \Gamma, \overline{\mathbf{Q}}_{l}\right) \otimes \mathcal{H}_{x_{s}}^{r_{1}} A \otimes \mathcal{H}_{x_{s}}^{r_{2}} A^{\prime}\right)^{G_{\underline{0}}\left(x_{s}\right) / G_{\underline{0}}\left(x_{s}\right)^{0},}
\end{aligned}
$$

where the upper script refers to invariants under the finite group $G_{\underline{0}}\left(x_{s}\right) / G_{0}\left(x_{s}\right)^{0}$.

(c) Assume that $\mathbf{k}$ is as in 12.1. Then $H_{c}^{a}\left(X_{\leq s}, \tilde{A} \otimes \tilde{A}^{\prime}\right)$ is a-pure (see 12.1) for $s=0,1, \ldots, \beta$ and $a \sim 2 \mathbf{D}$.

(d) The exact sequence 13.1(a) gives rise to short exact sequences

$$
0 \rightarrow H_{c}^{a}\left(X_{s}, \tilde{A} \otimes \tilde{A}^{\prime}\right) \rightarrow H_{c}^{a}\left(X_{\leq s}, \tilde{A} \otimes \tilde{A}^{\prime}\right) \rightarrow H_{c}^{a}\left(X_{\leq s-1}, \tilde{A} \otimes \tilde{A}^{\prime}\right) \rightarrow 0
$$

for $s=0,1, \ldots, \beta$ and $a \sim 2 \mathbf{D}$.

(e) For $a \sim 2 \mathbf{D}$ we have $\operatorname{dim} H_{c}^{a}\left(X, \tilde{A} \otimes \tilde{A}^{\prime}\right)=\sum_{s=0}^{\beta} \operatorname{dim} H_{c}^{a}\left(X_{s}, \tilde{A} \otimes \tilde{A}^{\prime}\right)$.

(f) For $a \sim 2 \mathbf{D}$ we have

$$
\begin{aligned}
& \operatorname{dim} H_{c}^{a}\left(X, \tilde{A} \otimes \tilde{A}^{\prime}\right) \\
& =\sum_{s=0}^{\beta} \sum_{r+r_{1}+r_{2}=a} \operatorname{dim}\left(H_{c}^{r}\left(G_{\underline{0}}\left(x_{s}\right)^{0} \backslash \Gamma, \overline{\mathbf{Q}}_{l}\right) \otimes \mathcal{H}_{x_{s}}^{r_{1}} A \otimes \mathcal{H}_{x_{s}}^{r_{2}} A^{\prime}\right)^{G_{0}\left(x_{s}\right) / G_{\underline{0}}\left(x_{s}\right)^{0} .}
\end{aligned}
$$

The proof is almost a copy of the proof of [L4, 19.4]. By general principles we can assume that $\mathbf{k}$ is as in 12.1. We shall use Deligne's theory of weights. We first prove (a) and (b). We write $x$ instead of $x_{s}$. We may assume that $x$ is an $\mathbf{F}_{q}$-rational point. We have a natural spectral sequence

$$
E_{2}^{r, r^{\prime}}=H_{c}^{r}\left(X_{s}, \mathcal{H}^{r^{\prime}}\left(\tilde{A} \otimes \tilde{A}^{\prime}\right)\right) \Longrightarrow H_{c}^{r+r^{\prime}}\left(X_{s}, \tilde{A} \otimes \tilde{A}^{\prime}\right) .
$$

We show that

$$
E_{2}^{r, r^{\prime}} \text { is }\left(r+r^{\prime}\right) \text {-pure if } r+r^{\prime} \sim 2 \mathbf{D} .
$$

We have $X_{s}=G_{\underline{0}}(x) \backslash \Gamma$ and

$$
E_{2}^{r, r^{\prime}}=\left(H_{c}^{r}\left(G_{\underline{0}}(x)^{0} \backslash \Gamma, \overline{\mathbf{Q}}_{l}\right) \otimes \mathcal{H}_{x}^{r^{\prime}}\left(A \otimes A^{\prime}\right)\right)^{G_{\underline{0}}(x) / G_{\underline{0}}(x)^{0}} .
$$


Here $A \otimes A^{\prime} \in \mathcal{D}\left(\mathfrak{g}_{\delta}\right)$. We may assume that $r^{\prime}$ is "small" (otherwise, $E_{2}^{r, r^{\prime}}=0$ ). We then have $r \sim 2 \mathbf{D}$. Now

$$
\mathcal{H}_{x}^{r^{\prime}}\left(A \otimes A^{\prime}\right)=\oplus_{r_{1}+r_{2}=r^{\prime}} \mathcal{H}_{x}^{r_{1}}(A) \otimes \mathcal{H}_{x}^{r_{2}}\left(A^{\prime}\right)
$$

is $r^{\prime}$-pure by $12.2(\mathrm{a})$. Moreover, $H_{c}^{r}\left(G_{\underline{0}}(x)^{0} \backslash \Gamma, \overline{\mathbf{Q}}_{l}\right.$ ) is $r$-pure for $r \sim 2 \mathbf{D}$ (a known property of the classifying space of $\left.G_{\underline{0}}(x)^{0}\right)$ and (h) follows.

From (h) it follows that $E_{\infty}^{r, r^{\prime}}$ of the spectral sequence $(\mathrm{g})$ is $\left(r+r^{\prime}\right)$-pure if $r+r^{\prime} \sim 2 \mathbf{D}$ and (a) follows. From (h) it also follows that $E_{2}^{r, r^{\prime}}=E_{\infty}^{r, r^{\prime}}$ if $r+r^{\prime} \sim 2 \mathbf{D}$ (many differentials must be zero since they respect weights) and (b) follows.

Now (c) follows from (a) using the exact sequence 13.2(a) and induction on $s$. From (c) and (a) we see that the homomorphism $\xi_{a+1}$ in 13.2(a) is between pure spaces of different weight. Since $\xi_{a+1}$ preserve weights, it must be 0 . Similarly, $\xi_{a}=0$ in 13.2(a) hence (d) holds. Now (e) follows from (d) since the support of $\tilde{A} \otimes \tilde{A}^{\prime}$ is contained in $X_{\leq \beta}$; (f) follows from (b),(e). This completes the proof of the proposition.

13.4. Using the definitions we see that 13.3(f) implies:

(a)

$d_{j}\left(\mathfrak{g}_{\delta} ; A, A^{\prime}\right)=\sum_{s} \sum_{-r_{0}+r_{1}+r_{2}=j-2 p_{s}} \operatorname{dim}\left(H_{r_{0}}^{G_{0}\left(x_{s}\right)^{0}}(\mathrm{pt}) \otimes \mathcal{H}_{x_{s}}^{r_{1}} A \otimes \mathcal{H}_{x_{s}}^{r_{2}} A^{\prime}\right)^{G_{\underline{0}}\left(x_{s}\right) / G_{\underline{0}}\left(x_{s}\right)^{0}}$,

where

$$
p_{s}=\operatorname{dim} G_{\underline{0}}-\operatorname{dim} G_{\underline{0}}\left(x_{s}\right)=\operatorname{dim} \mathcal{O}_{s}
$$

and $H_{r_{0}}^{G_{0}\left(x_{s}\right)^{0}}$ (pt) denotes equivariant homology of a point. (See [L6] for the definition of equivariant homology.)

13.5. Given $(\mathcal{O}, \mathcal{L}),(\tilde{\mathcal{O}}, \tilde{\mathcal{L}})$ in $\mathcal{I}\left(\mathfrak{g}_{\delta}\right)$ we define $\mu(\tilde{\mathcal{L}}, \mathcal{L}) \in \mathbf{Z}\left[v^{-1}\right]$ by

$$
\mu(\tilde{\mathcal{L}}, \mathcal{L})=\sum_{a} \mu(a ; \tilde{\mathcal{L}}, \mathcal{L}) v^{-a}
$$

where $\mu(a ; \tilde{\mathcal{L}}, \mathcal{L})$ is the number of times $\tilde{\mathcal{L}}$ appears in a decomposition of the local system $\left.\mathcal{H}^{a}\left(\mathcal{L}^{\sharp}\right)\right|_{\tilde{\mathcal{O}}}$ as a direct sum of irreducible local systems. Note that $\mu(\tilde{\mathcal{L}}, \mathcal{L})$ is zero unless $\tilde{\mathcal{O}}$ is contained in the closure of $\mathcal{O}$.

If $\mathcal{E}$ is an irreducible $G_{\underline{0}}$-equivariant local system on $\mathcal{O}_{s}$, we denote by $\rho_{\mathcal{E}}$ the irreducible $G_{\underline{0}}\left(x_{s}\right) / G_{\underline{0}}\left(x_{s}\right)^{\overline{0}}$-module corresponding to $\mathcal{E}$. With this notation we can rewrite $13.4(\overline{\mathrm{a}})$ as follows:

$$
\begin{aligned}
& d_{j}\left(\mathfrak{g}_{\delta} ; A, A^{\prime}\right)=\sum_{s} \sum_{-r_{0}+r_{1}+r_{2}=j-2 p_{s}} \\
& \sum_{\mathcal{E}, \mathcal{E}^{\prime}} \mu\left(r_{1} ; \mathcal{E}, \mathcal{L}\right) \mu\left(r_{2} ; \mathcal{E}^{\prime}, \mathcal{L}^{\prime}\right) \operatorname{dim}\left(H_{r_{0}}^{G_{0}}\left(x_{s}\right)^{0}(\mathrm{pt}) \otimes \rho_{\mathcal{E}} \otimes \rho_{\mathcal{E}^{\prime}}\right)^{G_{\underline{0}}\left(x_{s}\right) / G_{\underline{0}}\left(x_{s}\right)^{0},},
\end{aligned}
$$

where $\mathcal{E}, \mathcal{E}^{\prime}$ run over the isomorphism classes of irreducible $G_{\underline{0}}$-equivariant local systems on $\mathcal{O}_{s}$. This may be written in terms of power series in $\mathbf{Q}((v))$ as follows:

$$
\left\{A, A^{\prime}\right\}=\sum_{s=0}^{\beta} \sum_{\mathcal{E}, \mathcal{E}^{\prime}} \mu(\mathcal{E}, \mathcal{L}) \Xi\left(\mathcal{E}, \mathcal{E}^{\prime}\right) \mu\left(\mathcal{E}^{\prime}, \mathcal{L}^{\prime}\right),
$$


where

(c) $\Xi\left(\mathcal{E}, \mathcal{E}^{\prime}\right)=\sum_{r_{0} \geq 0} \operatorname{dim}\left(H_{r_{0}}^{G_{0}\left(x_{s}\right)^{0}}(\mathrm{pt}) \otimes \rho_{\mathcal{E}} \otimes \rho_{\mathcal{E}^{\prime}}\right)^{G_{\underline{0}}\left(x_{s}\right) / G_{\underline{0}}\left(x_{s}\right)^{0}} v^{r_{0}-2 p_{s}} \in \mathbf{Q}((v))$.

13.6. Let $B_{1}, B_{2} \in \mathfrak{B}$. We write $B_{1}=\mathcal{L}_{1}^{\sharp}\left[\operatorname{dim} \mathcal{O}_{1}\right] \in \mathcal{D}\left(\mathfrak{g}_{\delta}\right), B_{2}=\mathcal{L}_{2}^{\sharp}\left[\operatorname{dim} \mathcal{O}_{2}\right] \in$ $\mathcal{D}\left(\mathfrak{g}_{\delta}\right)$ with $\left(\mathcal{O}_{1}, \mathcal{L}_{1}\right),\left(\mathcal{O}_{2}, \mathcal{L}_{2}\right)$ in $\mathcal{I}\left(\mathfrak{g}_{\delta}\right)$. We set

$P_{B_{2}, B_{1}}=\mu\left(\mathcal{L}_{2}, \mathcal{L}_{1}\right) \in \mathbf{N}\left[v^{-1}\right]$, (see 13.5);

$\tilde{P}_{B_{2}, B_{1}}=P_{D\left(B_{2}\right), D\left(B_{1}\right)} \in \mathbf{N}\left[v^{-1}\right]$;

$\Lambda_{B_{2}, B_{1}}=\Xi\left(\mathcal{L}_{2}, \mathcal{L}_{1}\right) \in \mathbf{Q}((v)),($ see 13.5$)$ if $\mathcal{O}_{1}=\mathcal{O}_{2} ;$

$\Lambda_{B_{2}, B_{1}}=0$ if $\mathcal{O}_{1} \neq \mathcal{O}_{2}$;

$\tilde{\Lambda}_{B_{2}, B_{1}}=\Lambda_{B_{2}, D\left(B_{1}\right)} \in \mathbf{Q}((v))$.

Note that:

(a) $P_{B_{2}, B_{1}} \neq 0 \Longrightarrow \operatorname{dim} \mathcal{O}_{2} \leq \operatorname{dim} \mathcal{O}_{1} ; \tilde{P}_{B_{2}, B_{1}} \neq 0 \Longrightarrow \operatorname{dim} \mathcal{O}_{2} \leq \operatorname{dim} \mathcal{O}_{1}$;

(b) if $\operatorname{dim} \mathcal{O}_{2}=\operatorname{dim} \mathcal{O}_{1}$, then $P_{B_{2}, B_{1}}=\delta_{B_{2}, B_{1}}, \tilde{P}_{B_{2}, B_{1}}=\delta_{B_{2}, B_{1}}$;

(c) $\tilde{\Lambda}_{B_{2}, B_{1}}=0$ if $\mathcal{O}_{1} \neq \mathcal{O}_{2}$.

Then $13.5(\mathrm{~b})$ can be rewritten as follows:

$$
\left\{A, A^{\prime}\right\}=\sum_{B_{1} \in \mathfrak{B}, B_{2} \in \mathfrak{B}} P_{B_{1}, B} \Lambda_{B_{1}, B_{2}} P_{B_{2}, B^{\prime \prime}},
$$

where $B=A[\operatorname{dim} \mathcal{O}], B^{\prime \prime}=A^{\prime}\left[\operatorname{dim} \mathcal{O}^{\prime}\right]$, or as

$$
\left\{A, A^{\prime}\right\}=\sum_{B_{1} \in \mathfrak{B}, B_{2} \in \mathfrak{B}} P_{B_{1}, B} \Lambda_{B_{1}, D\left(B_{2}\right)} P_{D\left(B_{2}\right), D\left(B^{\prime}\right)},
$$

where $B=A[\operatorname{dim} \mathcal{O}], D\left(B^{\prime}\right)=A^{\prime}\left[\operatorname{dim} \mathcal{O}^{\prime}\right]$, or as

$$
\left\{A, A^{\prime}\right\}=\sum_{B_{1} \in \mathfrak{B}, B_{2} \in \mathfrak{B}} P_{B_{1}, B} \tilde{\Lambda}_{B_{1}, B_{2}} \tilde{P}_{B_{2}, B^{\prime}}
$$

where $B=A[\operatorname{dim} \mathcal{O}], D\left(B^{\prime}\right)=A^{\prime}\left[\operatorname{dim} \mathcal{O}^{\prime}\right]$. We have

$$
\left\{A, A^{\prime}\right\}=v^{-\kappa(B)-\kappa\left(B^{\prime}\right)}\left\{B, D\left(B^{\prime}\right)\right\}=v^{-\kappa(B)-\kappa\left(B^{\prime}\right)}\left(B: B^{\prime}\right),
$$

hence

$$
v^{-\kappa(B)-\kappa\left(B^{\prime}\right)}\left(B: B^{\prime}\right)=\sum_{B_{1} \in \mathfrak{B}, B_{2} \in \mathfrak{B}} P_{B_{1}, B} \tilde{\Lambda}_{B_{1}, B_{2}} \tilde{P}_{B_{2}, B^{\prime}}
$$

for any $B, B^{\prime}$ in $\mathfrak{B}$. (Here $\kappa$ is as in 10.13.) We show:

(e) The following three matrices with entries in $\mathbf{Q}((v)$ ) (indexed by $\mathfrak{B} \times \mathfrak{B}$ ) are invertible:

(i) the matrix $\left(\left(B: B^{\prime}\right)\right)$;

(ii) the matrix $\mathcal{M}:=\left(v^{-\kappa(B)-\kappa\left(B^{\prime}\right)}\left(B: B^{\prime}\right)\right)$;

(iii) the matrix $\mathcal{T}:=\left(\tilde{\Lambda}_{B, B^{\prime}}\right)$.

The matrix in (i) is invertible since $\left(B: B^{\prime}\right) \in \delta_{B, B^{\prime}}+v \mathbf{N}[[v]]$ for all $B, B^{\prime}$; see [LY, 0.12]. This implies immediately that $\mathcal{M}$ is invertible. Now, by (d), we have $\mathcal{M}=\mathcal{S} \mathcal{T} \mathcal{S}^{\prime}$ where $\mathcal{S}$ (resp. $\left.\mathcal{S}^{\prime}\right)$ is the matrix indexed by $\mathfrak{B} \times \mathfrak{B}$ whose $\left(B, B^{\prime}\right)$-entry is $P_{B^{\prime}, B}$ (resp. $\tilde{P}_{B, B^{\prime}}$ ). Since $\mathcal{M}$ is invertible, it follows that $\mathcal{T}$ is invertible. This proves (e). 
13.7. We show:

(a) If $B, B^{\prime}$ in $\mathfrak{B}$ satisfy $\psi(B) \neq \psi\left(B^{\prime}\right)$ ( $\psi$ as in $\left[\mathrm{LY}\right.$, 6.6]), then $P_{B, B^{\prime}}=0$, $\tilde{P}_{B, B^{\prime}}=0, \tilde{\Lambda}_{B, B^{\prime}}=0$.

We can find a function $\mathfrak{B} \rightarrow \mathbf{Z}, B \mapsto c_{B}$ such that for $B, B^{\prime}$ in $\mathfrak{B}$ we have $c_{B}=c_{B^{\prime}}$ if and only if $\psi(B)=\psi\left(B^{\prime}\right)$. From 13.6 we deduce

$$
\begin{aligned}
& v^{c_{B}-c_{B^{\prime}}} v^{-\kappa(B)-\kappa\left(B^{\prime}\right)}\left(B: B^{\prime}\right) \\
& =\sum_{B_{1} \in \mathfrak{B}, B_{2} \in \mathfrak{B}} v^{c_{B}-c_{B_{1}}} P_{B_{1}, B} v^{c_{B_{1}}-c_{B_{2}}} \tilde{\Lambda}_{B_{1}, B_{2}} v^{c_{B_{2}}-c_{B^{\prime}}} \tilde{P}_{B_{2}, B^{\prime}} .
\end{aligned}
$$

When $B, B^{\prime}$ vary, this again can be expressed as the decomposition of the matrix $\tilde{\mathcal{M}}:=\left(v^{c_{B}-c_{B^{\prime}}} v^{-\kappa(B)-\kappa\left(B^{\prime}\right)}\left(B: B^{\prime}\right)\right)$ (indexed by $\mathfrak{B} \times \mathfrak{B}$ ) as a product of three matrices $\tilde{\mathcal{S}} \tilde{\mathcal{T}} \tilde{\mathcal{S}}^{\prime}$, where $\tilde{\mathcal{S}}$ (resp. $\tilde{\mathcal{S}}^{\prime}$ ) is the matrix indexed by $\mathfrak{B} \times \mathfrak{B}$ whose $\left(B, B^{\prime}\right)$ entry is $v^{c_{B}-c_{B^{\prime}}} P_{B^{\prime}, B}$ (resp. $\left.v^{c_{B}-c_{B^{\prime}}} \tilde{P}_{B, B^{\prime}}\right)$ and $\tilde{\mathcal{T}}$ is the matrix indexed by $\mathfrak{B} \times \mathfrak{B}$ whose $\left(B, B^{\prime}\right)$-entry is $v^{c_{B}-c_{B^{\prime}}} \tilde{\Lambda}_{B, B^{\prime}}$. From [LY, $\left.7.9(\mathrm{a})\right]$ we know that $\left(B: B^{\prime}\right)=0$ unless $\psi(B)=\psi\left(B^{\prime}\right)$. Hence $v^{c_{B}-c_{B^{\prime}}}\left(B: B^{\prime}\right)=\left(B: B^{\prime}\right)$ for all $B, B^{\prime}$ so that $\tilde{\mathcal{M}}=\mathcal{M}$. Thus we have

$$
\tilde{\mathcal{S}} \tilde{\mathcal{T}} \tilde{\mathcal{S}}^{\prime}=\mathcal{S} \mathcal{T} \mathcal{S}^{\prime}
$$

Now by 13.6(c),(e), the matrix $\mathcal{T}$ (and hence the matrix $\tilde{\mathcal{T}}$ ) belongs to a subgroup of $G L_{N}(N=\sharp(\mathfrak{B}))$ of the form $G L_{N_{1}} \times \cdots \times G L_{N_{k}}$ where $N_{1}, \ldots, N_{k}$ are the sizes of the various subsets $\mathfrak{B}_{\mathcal{O}}$; moreover, by 13.6(a),(b), the matrix $\mathcal{S}$ (hence the matrix $\tilde{\mathcal{S}}$ ) belongs to the unipotent radical of a parabolic subgroup of $G L_{N}$ with Levi subgroup equal to the subgroup of $G L_{N}$ considered above, while the matrix $\mathcal{S}^{\prime}$ (hence the matrix $\tilde{\mathcal{S}}^{\prime}$ ) belongs to the unipotent radical of the opposite parabolic subgroup with the same Levi subgroup. This forces the equalities $\tilde{\mathcal{S}}=\mathcal{S}, \tilde{\mathcal{T}}=\mathcal{T}$, $\tilde{\mathcal{S}}^{\prime}=\mathcal{S}^{\prime}$. Now the equality $\tilde{\mathcal{S}}=\mathcal{S}$ implies $v^{c_{B}-c_{B^{\prime}}} P_{B^{\prime}, B}=P_{B^{\prime}, B}$ for all $B^{\prime}, B$ in $\mathfrak{B}$. Thus, if $\psi(B) \neq \psi\left(B^{\prime}\right)$ (so that $c_{B} \neq c_{B^{\prime}}$ ), we must have $P_{B^{\prime}, B}=0$. Similarly, from $\tilde{\mathcal{T}}=\mathcal{T}$ we see that, if $\psi(B) \neq \psi\left(B^{\prime}\right)$, then $\tilde{\Lambda}_{B, B^{\prime}}=0$ and from $\tilde{\mathcal{S}}^{\prime}=\mathcal{S}^{\prime}$ we see that, if $\psi(B) \neq \psi\left(B^{\prime}\right)$, then $\tilde{P}_{B, B^{\prime}}=0$. This proves (a).

13.8. We now fix $\xi \in \underline{\mathfrak{T}}_{\eta}$. We define four matrices ${ }^{\xi} \mathcal{M},{ }^{\xi} \mathcal{S},{ }^{\xi} \mathcal{T},{ }^{\xi} \mathcal{S}^{\prime}$ indexed by $\xi_{\mathfrak{B}} \times \xi_{\mathfrak{B}}$ as follows. For $B, B^{\prime}$ in $\xi_{\mathfrak{B}}$, the $\left(B, B^{\prime}\right)$-entry of $\xi_{\mathcal{M}}$ is $v^{-\kappa(B)-\kappa\left(B^{\prime}\right)}(B$ : $\left.B^{\prime}\right)$; the $\left(B, B^{\prime}\right)$-entry of ${ }^{\xi} \mathcal{S}$ is $P_{B^{\prime}, B}$; the $\left(B, B^{\prime}\right)$-entry of ${ }^{\xi} \mathcal{T}$ is $\tilde{\Lambda}_{B, B^{\prime}}$; the $\left(B, B^{\prime}\right)$ entry of ${ }^{\xi} \mathcal{S}^{\prime}$ is $\tilde{P}_{B, B^{\prime}}$. Using 13.7 (a) we deduce from $13.6(\mathrm{~d})$ the equality of matrices

$$
{ }^{\xi} \mathcal{M}=\left({ }^{\xi} \mathcal{S}\right)\left({ }^{\xi} \mathcal{T}\right)\left({ }^{\xi} \mathcal{S}^{\prime}\right)
$$

As in the proof of 13.7 (a) the last equality determines uniquely the matrices ${ }^{\xi} \mathcal{S},{ }^{\xi} \mathcal{T}$, $\xi^{\xi} \mathcal{S}^{\prime}$ if the matrix ${ }^{\xi} \mathcal{M}$ is known; in fact, it provides an algorithm for computing the entries of these three matrices (and in particular for the entries $P_{B^{\prime}, B}$ in terms of the entries of $\xi^{\mathcal{M}}$. Now under the bijection $\gamma: \mathbf{B} \stackrel{\sim}{\longrightarrow} \xi_{\mathfrak{B}}$ (see 11.12(a)) the matrix $\xi_{\mathcal{M}}$ becomes a matrix indexed by $\mathbf{B} \times \mathbf{B}$ whose $\left(b, b^{\prime}\right)$-entry is $v^{-\kappa(B)-\kappa\left(B^{\prime}\right)}\left(b, b^{\prime}\right)$; these entries are explicitly computable from the combinatorial description of $(:)$ on V. We see that:

(b) The quantities $P_{B^{\prime}, B}$ are computable by an algorithm provided that the bijection $\gamma: \mathbf{B} \stackrel{\sim}{\longrightarrow} \xi_{\mathfrak{B}}$ is known.

This can be used in several examples to compute the $P_{B^{\prime}, B}$ explicitly. The algorithm in (b) seems to depend on the choice of $\eta$ such that $\eta=\delta$; but in fact, by the results in [LY, 3.9], 10.22, 11.13, it does not depend on this choice. 
In the case where $m=1$, there is another algorithm to compute the quantities $P_{B^{\prime}, B}$; see [L7, Theorem 24.8]. It again displays the matrix ${ }^{\xi} \mathcal{S}$ as the first of the three factors in a matrix decomposition like (a), but with the matrix ${ }^{\xi} \mathcal{M}$ being replaced by a matrix indexed by a pair of irreducible representation of a Weyl group and with entries determined by a prescription quite different from that used in this paper. In that case the bijection $\gamma: \mathbf{B} \stackrel{\sim}{\longrightarrow} \xi \mathfrak{B}$ is replaced by the "generalized Springer correspondence". It would be interesting to understand better the connection between these two approaches to the quantities $P_{B^{\prime}, B}$.

\section{ODD VANISHING}

In this section we show that for any irreducible local system $\mathcal{L}$ on a $G_{\underline{0}}$-orbit in $\mathfrak{g}_{\delta}^{\text {nil }}$ the cohomology sheaves of $\mathcal{L}^{\sharp} \in \mathcal{D}\left(\mathfrak{g}_{\delta}\right)$ are zero in odd degrees. (See Theorem 14.10.) In the case where $m \gg 0$, this follows from the analogous result in the $\mathbf{Z}$-graded case in [L4]. In the case where $m=1$ this follows from [L7, Theorem 24.8(a)]. In the case where $m>1, \delta=\underline{1}, \mathcal{L}=\overline{\mathbf{Q}}_{l}$ and $G, \mathfrak{g}=\oplus_{i} \mathfrak{g}_{i}$ are as in [LY, 0.3], this follows from [L8, Theorem 11.3] and from the known odd vanishing result for affine Schubert varieties.

14.1. We preserve the setup of 10.1, 10.2. For $\varpi \in \mathbf{E}^{\prime}$ let $h(\varpi) \in \mathbf{Z}$ be as in 10.2. For $\mathbf{c} \in \stackrel{\circ}{\mathbf{E}}$ we set $h(\mathbf{c})=h(\varpi)$ where $\varpi$ is any element of $\mathbf{c} \cap \mathbf{E}^{\prime}$; this is well defined, by $10.7(\mathrm{a})$. For $\mathbf{c} \in \underline{\circ} \underline{\mathbf{E}}$ we set $T_{\mathbf{c}}=v^{-h(\mathbf{c})} \tilde{T}_{\mathbf{c}} \in \mathbf{V}^{\prime}$. Note that $\left\{T_{\mathbf{c}} ; \mathbf{c} \in \stackrel{\circ}{\mathbf{E}}\right\}$ is a $\mathbf{Q}(v)$-basis of $\mathbf{V}^{\prime}$. Let $f \mapsto f^{\odot}$ be the field involution of $\mathbf{Q}(v)$ which carries $v$ to $-v$; this extends to a field involution of $\mathbf{Q}((v))$ (denoted again by $f \mapsto f^{\odot}$ ) given by $\sum_{i} c_{i} v^{i} \rightarrow \sum_{i} c_{i}(-v)^{i}$, where $c_{i} \in \mathbf{Q}$.

Let $b \mapsto b^{\odot}$ be the Q-linear involution $\mathbf{V}^{\prime} \rightarrow \mathbf{V}^{\prime}$ such that $\left(f T_{\mathbf{c}}\right)^{\odot}=f^{\odot} T_{\mathbf{c}}$ for any $\mathbf{c} \in \underline{\stackrel{\circ}{\mathbf{E}}}$ and $f \in \mathbf{Q}(v)$.

Lemma 14.2 (14.2). For any $b, b^{\prime}$ in $\mathbf{V}^{\prime}$ we have $\left(b^{\odot}: b^{\prime \odot}\right)=\left(b: b^{\prime}\right)^{\curvearrowright}$.

It is enough to show that for any $\mathbf{c}, \mathbf{c}^{\prime}$ in $\underline{\circ}$ and any $f, f^{\prime}$ in $\mathbf{Q}(v)$ we have

$$
\left(f^{\odot} T_{\mathbf{c}}: f^{\prime} T_{\mathbf{c}^{\prime}}\right)=\left(f T_{\mathbf{c}}: f^{\prime} T_{\mathbf{c}^{\prime}}\right)^{\ominus} \text {. }
$$

We can assume that $f=f^{\prime}=1$. It is enough to show that

$$
\left(T_{\mathbf{c}}: T_{\mathbf{c}^{\prime}}\right) \in \mathbf{Q}\left(v^{2}\right),
$$

or that

$$
v^{-h\left(\varpi_{1}\right)+h\left(\varpi_{2}\right)}\left[\varpi_{1} \mid \varpi_{2}\right] \in \mathbf{Q}\left(v^{2}\right)
$$

for any $\varpi_{1}, \varpi_{2}$ in $\mathbf{E}^{\prime}$ (notation of $10.12($ a)) or that

$$
v^{-h\left(\varpi_{1}\right)+h\left(\varpi_{2}\right)} \sum_{w \in \mathcal{W}} v^{\tau\left(\varpi_{2}, w \varpi_{1}\right)} \in \mathbf{Q}\left(v^{2}\right) .
$$

From 10.8 (b) we see that for $\varpi, \varpi^{\prime}$ in $\mathbf{E}^{\prime}$ we have

$$
\tau\left(\varpi, \varpi^{\prime}\right)=h(\varpi)+h\left(\varpi^{\prime}\right) \bmod 2 .
$$

From 10.11(b) we see that for $\varpi \in \mathbf{E}^{\prime}, w \in \mathcal{W}$ and $N \in \mathbf{Z}$ we have

$$
\operatorname{dim} \epsilon_{\mathfrak{p}} \frac{w \varpi}{N}=\operatorname{dim} \epsilon_{\mathfrak{p}} \frac{\varpi}{N} \text {, hence } \operatorname{dim} \epsilon_{\mathfrak{u}} \frac{w \varpi}{N}=\operatorname{dim}{ }^{\epsilon} \mathfrak{u}_{N},
$$

so that

$$
h(w \varpi)=h(\varpi) .
$$


Using this and (b) we see that for $\varpi, \varpi^{\prime}$ in $\mathbf{E}^{\prime}$ we have

$$
\sum_{w \in \mathcal{W}} v^{\tau\left(\varpi^{\prime}, w \varpi\right)} \in v^{h\left(\varpi^{\prime}\right)+h(\varpi)} \mathbf{N}\left[v^{2}, v^{-2}\right] .
$$

Thus, (b) holds. The lemma is proved.

14.3. From 14.2 we deduce that ${ }^{\curvearrowright}: \mathbf{V}^{\prime} \rightarrow \mathbf{V}^{\prime}$ carries $\mathfrak{R}_{l}=\mathfrak{R}_{r}$ onto $\mathfrak{R}_{l}=\mathfrak{R}_{r}$; hence it induces a $\mathbf{Q}$-linear involution $\mathbf{V} \rightarrow \mathbf{V}\left(\right.$ denoted again by $\left.^{\circledR}\right)$. From 14.2 we deduce:

(a) For any $b, b^{\prime}$ in $\mathbf{V}$ we have $\left(b^{\odot}: b^{\prime}\right)=\left(b: b^{\prime}\right)^{\odot}$.

14.4. Let $\mathbf{c} \in \underline{\mathbf{E}}, \varpi \in \mathbf{E}^{\prime \prime} \cap \mathbf{c}$ and let $a_{\varpi}=\sum_{j} v^{-2 s_{j}} v^{d(\varpi)} \in \mathcal{A}$ be as in 11.1. Note that

$$
\left(a_{\varpi}\right)^{\bigodot}=\sum_{j} v^{-2 s_{j}}(-v)^{d(\varpi)}=(-1)^{d(\varpi)} a_{\varpi} .
$$

Hence we have

$$
\left(a_{\varpi}^{-1} \tilde{T}_{\mathbf{c}}\right)^{\odot}=\left(a_{\varpi}^{-1} v^{h(\mathbf{c})} T_{\mathbf{c}}\right)^{\odot}=(-1)^{d(\varpi)+h(\mathbf{c})} a_{\varpi}^{-1} v^{h(\mathbf{c})} T_{\mathbf{c}}==(-1)^{d(\varpi)+h(\mathbf{c})} a_{\varpi}^{-1} \tilde{T}_{\mathbf{c}} .
$$

It follows that:

(a) ${ }^{\odot}: \mathbf{V}^{\prime} \rightarrow \mathbf{V}^{\prime}$ carries $\mathbf{V}_{\mathcal{A}}^{\prime}$ onto $\mathbf{V}_{\mathcal{A}}^{\prime}$ and ${ }^{\odot}: \mathbf{V} \rightarrow \mathbf{V}$ carries $\mathbf{V}_{\mathcal{A}}$ onto $\mathbf{V}_{\mathcal{A}}$.

14.5. We show:

a) For any $b \in \mathbf{V}^{\prime}$ we have $\overline{b^{\odot}}=(\bar{b})^{\odot}$. Hence for any $b \in \mathbf{V}$ we have $\overline{b^{\odot}}=(\bar{b})^{\odot}$.

We can assume that $f=f T_{\mathbf{c}}$ where $\mathbf{c} \in \underline{\mathbf{E}}$ and $f \in \mathbf{Q}(v)$. Note that $\overline{f^{\odot}}=(\bar{f})^{\odot}$. Hence we can assume that $f=1$. We have

$$
\overline{T_{\mathbf{c}}}=\overline{v^{-h(\mathbf{c})} \tilde{T}_{\mathbf{c}}}=v^{h(\mathbf{c})} \tilde{T}_{\mathbf{c}}=v^{2 h(\mathbf{c})} T_{\mathbf{c}}
$$

hence

$$
\left(\overline{T_{\mathbf{c}}}\right)^{\odot}=v^{2 h(\mathbf{c})} T_{\mathbf{c}}
$$

We have $\overline{T_{\mathbf{c}}^{\odot}}=\overline{T_{\mathbf{c}}}=v^{2 h(\mathbf{c})} T_{\mathbf{c}}$. This proves (a).

14.6. We show:

(a) $b \mapsto b^{\odot}$ is a bijection $\mathbf{B}^{\prime} \stackrel{\sim}{\longrightarrow} \mathbf{B}^{\prime}$.

Let $b \in \mathbf{B}^{\prime}$. From $b \in \mathbf{V}_{\mathcal{A}}$ we see using $14.4(\mathrm{a})$ that $b^{\odot} \in \mathbf{V}_{\mathcal{A}}$. From $\bar{b}=b$ we see using 14.5(a) that $\overline{b^{\odot}}=(\bar{b})^{\odot}=b^{\odot}$. From $(b: b) \in \mathbf{Q}(v) \cap(1+v \mathbf{Z}[[v]])$ we see using 14.3(a) that

$\left(b^{\odot}: b^{\prime \odot}\right)=\left(b: b^{\prime}\right)^{\odot} \in(\mathbf{Q}(v) \cap(1+v \mathbf{Z}[[v]]))^{\odot}=\mathbf{Q}(v) \cap(1+v \mathbf{Z}[[v]]) \subset 1+v \mathbf{Z}[[v]]$.

Using this and the definitions, we see that $b^{\curvearrowright} \in \mathbf{B}^{\prime}$. Thus the map $b \mapsto b^{\curvearrowright}, \mathbf{B}^{\prime} \rightarrow \mathbf{B}^{\prime}$ is well defined. Since this map has square 1, it is a bijection. This proves (a).

From (a) we deduce: $\tilde{b} \in \mathbf{B}$.

(b) If $b \in \mathbf{B}$, then $b^{\odot}=s_{b} \tilde{b}$ for a well-defined $s_{b} \in\{1,-1\}$ and a well-defined

The following result makes (b) more precise.

Lemma 14.7 (14.7). Let $\mathcal{O}$ be a $G_{\underline{0}}$-orbit in $\mathfrak{g}_{\delta}^{\text {nil }}$ and let $b \in \mathbf{B}_{\mathcal{O}}$. We have $b^{\curvearrowright}=(-1)^{\operatorname{dim} \mathcal{O}} b$. 
We argue by induction on $\operatorname{dim} \mathcal{O}$; we can assume that the result holds when $\mathcal{O}$ is replaced by an orbit of dimension $<\operatorname{dim} \mathcal{O}$ (if any). We have $\gamma(b)=\mathcal{L}^{\sharp}[\operatorname{dim} \mathcal{O}]$ where $(\mathcal{O}, \mathcal{L}) \in \mathcal{I}\left(\mathfrak{g}_{\delta}\right)$. Let $x \in \mathcal{O}$; we associate to $x$ an $\epsilon$-spiral $\mathfrak{p}_{*}^{\phi}$ and a splitting $\tilde{\mathfrak{l}}_{*}^{\phi}$ of it as in [LY, 2.9]. Recall that $\stackrel{\circ}{\mathfrak{l}}_{\eta} \subset \mathcal{O}$ and that $\mathcal{L}_{1}:=\left.\mathcal{L}\right|_{\tilde{\mathfrak{l}}_{\eta} \phi}$ is an irreducible $\tilde{L}_{0}^{\phi}$ equivariant local system on $\stackrel{\circ}{\mathfrak{l}}_{\eta}^{\phi}$; thus, $\mathcal{L}_{1}^{\sharp} \in \mathcal{D}\left(\tilde{\mathfrak{l}}_{\eta}^{\phi}\right)$ is defined. Let $I={ }^{\epsilon} \operatorname{Ind}_{\mathfrak{p}_{\eta}^{\phi}}^{\mathfrak{g}_{\delta}}\left(\mathcal{L}_{1}^{\sharp}\right) \in$ $\mathcal{Q}\left(\mathfrak{g}_{\delta}\right)$; we have clearly $I \in{ }^{\xi} \mathcal{Q}\left(\mathfrak{g}_{\delta}\right)$. By $[\mathrm{LY}, 2.9(\mathrm{~b}),(\mathrm{c})]$, in ${ }^{\xi} \mathcal{K}\left(\mathfrak{g}_{\delta}\right)$ we have

$$
I=\mathcal{L}^{\sharp}+\sum_{\mathcal{O}^{\prime} ; \operatorname{dim} \mathcal{O}^{\prime}<\operatorname{dim} \mathcal{O}} \sum_{b^{\prime} \in \mathbf{B}_{\mathcal{O}^{\prime}}} f_{b^{\prime}} \gamma\left(b^{\prime}\right),
$$

where $f_{b^{\prime}} \in \mathcal{A}$. Define $I^{\prime} \in \mathbf{V}_{\mathcal{A}}$ by $\gamma\left(I^{\prime}\right)=I$. We have

$$
I^{\prime}=v^{-\operatorname{dim} \mathcal{O}} b+\sum_{\mathcal{O}^{\prime} ; \operatorname{dim} \mathcal{O}^{\prime}<\operatorname{dim} \mathcal{O}} \sum_{b^{\prime} \in \mathbf{B}_{\mathcal{O}^{\prime}}} f_{b^{\prime}} b^{\prime} .
$$

By [L4, 17.2, 17.3], in $\mathcal{K}\left(\tilde{\mathfrak{l}}_{\eta}^{\phi}\right)$ we have

$$
h \mathcal{L}_{1}^{\sharp}=\sum_{j \in J} h_{j} \operatorname{ind}_{\mathfrak{q}(j)_{\eta}}^{\tilde{\mathfrak{l}}_{\eta}^{\phi}}(C(j)),
$$

where $h=h^{\curvearrowright} \in \mathcal{A}-\{0\}, J$ is a finite set and for $j \in J, \mathfrak{q}(j)$ is a parabolic subalgebra of $\tilde{\mathfrak{l}}_{\eta}^{\phi}$ with Levi subalgebra $\mathfrak{m}(j)$ such that $\mathfrak{q}(j), \mathfrak{m}(j)$ are compatible with the Z-grading of $\tilde{\mathfrak{l}}^{\phi}, C(j) \in \mathcal{Q}\left(\mathfrak{m}(j)_{\eta}\right)$ is a cuspidal perverse sheaf and $h_{j} \in \mathcal{A}$. Moreover, since $\mathcal{L}_{1}^{\sharp}$ belongs to the block of $\mathcal{Q}\left(\tilde{\mathfrak{l}}_{\eta}^{\phi}\right)$ given by $\xi$, we can assume that $\mathfrak{m}(j)=\mathfrak{m}$ and $C(j)=\tilde{C}$ for all $j$. Thus (b) can be written in the form

$$
h \mathcal{L}_{1}^{\sharp}=F_{0}+F_{1},
$$

where

$$
\begin{gathered}
\left.F_{0}=\sum_{j \in J} h_{j}^{\prime} \operatorname{ind}_{\mathfrak{q}(j)_{\eta}}^{\tilde{\mathfrak{l}}_{\eta}^{\phi}}\left(\tilde{C}\left[-\operatorname{dim} \mathfrak{m}_{\eta}\right]\right)\right), \\
\left.F_{1}=\sum_{j \in J} h_{j}^{\prime \prime} \operatorname{ind}_{\mathfrak{q}(j)_{\eta}}^{\tilde{\mathfrak{l}}_{\eta}^{\phi}}\left(\tilde{C}\left[-\operatorname{dim} \mathfrak{m}_{\eta}\right]\right)\right), \\
h_{j}^{\prime}+h_{j}^{\prime \prime}=h_{j} v^{\operatorname{dim} \mathfrak{m}_{\eta}}, h_{j}^{\prime} \in \mathbf{Z}\left[v^{2}, v^{-2}\right], h_{j}^{\prime \prime} \in v \mathbf{Z}\left[v^{2}, v^{-2}\right] .
\end{gathered}
$$

Let $\mathcal{K}\left(\tilde{\mathfrak{l}}_{\eta}^{\phi}\right)^{e v}$ be the $\mathbf{Z}\left[v^{2}, v^{-2}\right]$-submodule of $\mathcal{K}\left(\tilde{\mathfrak{l}}_{\eta}^{\phi}\right)^{e v}$ with basis $\mathcal{L}^{\prime \sharp}$ for various $\left(\mathcal{O}^{\prime}, \mathcal{L}^{\prime}\right)$ in $\mathcal{I}\left(\tilde{\mathfrak{l}} \eta_{\eta}\right)$. By [L4, 21.1(c)], for any $j \in J$, we have

$$
\operatorname{ind}_{\mathfrak{q}(j)_{\eta}}^{\tilde{\mathfrak{l}}_{\eta}^{\phi}}\left(\tilde{C}\left[-\operatorname{dim} \mathfrak{m}_{\eta}\right]\right) \in \mathcal{K}\left(\tilde{\mathfrak{l}}_{\eta}^{\phi}\right)^{e v} .
$$

Hence $F_{0} \in \mathcal{K}\left(\tilde{\mathfrak{l}}_{\eta}^{\phi}\right)^{e v}, F_{1} \in v \mathcal{K}\left(\tilde{\mathfrak{l}}_{\eta}^{\phi}\right)^{e v}$. We have clearly $h \mathcal{L}_{1}^{\sharp} \in \mathcal{K}\left(\tilde{\mathfrak{l}}_{\eta}^{\phi}\right)^{e v}$. Since $v \mathcal{K}\left(\tilde{\mathfrak{l}}_{\eta}^{\phi}\right)^{e v} \cap \mathcal{K}\left(\tilde{\mathfrak{l}}_{\eta}^{\phi}\right)^{e v}=0$, we deduce from (c) that $h \mathcal{L}_{1}^{\sharp}=F_{0}$; that is,

$$
\left.h \mathcal{L}_{1}^{\sharp}=\sum_{j \in J} h_{j}^{\prime} \operatorname{ind}_{\mathfrak{q}(j)_{\eta}}^{\tilde{\mathfrak{l}}_{\eta}^{\phi}}\left(\tilde{C}\left[-\operatorname{dim} \mathfrak{m}_{\eta}\right]\right)\right),
$$


where $h_{j}^{\prime} \in \mathbf{Z}\left[v^{2}, v^{-2}\right]$. Applying ${ }^{\epsilon} \operatorname{Ind}_{\mathfrak{p}_{\eta}}^{\mathfrak{g}_{\delta}}$ and using the transitivity property 4.2 from [LY] (and also 10.4(a)) we obtain

$$
h I=\sum_{j \in J} h_{j}^{\prime} I_{\varpi_{j}},
$$

where $\varpi_{j} \in \mathbf{E}^{\prime}$ for $j \in J$. Since $\gamma^{-1}\left(\sum_{j \in J} h_{j}^{\prime} I_{\varpi_{j}}\right)$ is fixed by ${ }^{\odot}$, it follows that $h I^{\prime}$ is fixed by ${ }^{\odot}$. Since $h \neq 0$ and $h^{\odot}=h$, we deduce that $I^{\prime \odot}=I^{\prime}$. Using (a) and the induction hypothesis, we see that

$$
\begin{aligned}
& \left(v^{-\operatorname{dim} \mathcal{O}} b\right)^{\mathcal{O}}+\sum_{\mathcal{O}^{\prime} ; \operatorname{dim} \mathcal{O}^{\prime}<\operatorname{dim} \mathcal{O}} \sum_{b^{\prime} \in \mathbf{B}_{\mathcal{O}^{\prime}}} f_{b^{\prime}}^{\odot}(-1)^{\operatorname{dim} \mathcal{O}^{\prime}} b^{\prime} \\
& =v^{-\operatorname{dim} \mathcal{O}} b+\sum_{\mathcal{O}^{\prime} ; \operatorname{dim} \mathcal{O}^{\prime}<\operatorname{dim} \mathcal{O}} \sum_{b^{\prime} \in \mathbf{B}_{\mathcal{O}^{\prime}}} f_{b^{\prime}} b^{\prime} .
\end{aligned}
$$

Using this and 14.6(b), we deduce that $(-v)^{-\operatorname{dim} \mathcal{O}_{s}} s_{b}-v^{-\operatorname{dim} \mathcal{O}_{b}}$ is a $\mathcal{A}$-linear combination of elements in $\cup_{\mathcal{O}^{\prime} ; \operatorname{dim} \mathcal{O}^{\prime}<\operatorname{dim} \mathcal{O}} \mathbf{B}_{\mathcal{O}^{\prime}}$.

Since $b \in \mathbf{B}_{\mathcal{O}}$ and $\tilde{b} \in \mathbf{B}$ this forces $\tilde{b}=b$ and $s_{b}=(-1)^{\operatorname{dim} \mathcal{O}}$. This completes the proof of the lemma.

14.8. We show:

(a) Let $\mathcal{O}, \mathcal{O}^{\prime}$ be $G_{\underline{0}}$-orbits in $\mathfrak{g}_{\delta}^{\text {nil }}$ and let $B, B^{\prime}$ in $\mathfrak{B}$ be such that the support of $B$ (resp. $\left.B^{\prime}\right)^{-}$is the closure of $\mathcal{O}$ (resp. $\left.\mathcal{O}^{\prime}\right)$. We have $\left(B: B^{\prime}\right)^{\mathcal{O}}=$ $(-1)^{\operatorname{dim} \mathcal{O}+\operatorname{dim} \mathcal{O}^{\prime}}\left(B: B^{\prime}\right)$.

If $\psi(B) \neq \psi\left(B^{\prime}\right)$, then $\left(B: B^{\prime}\right)=0$ by [LY, $\left.7.9(\mathrm{a})\right]$ and (a) holds. Assume now that $\psi(B)=\psi\left(B^{\prime}\right)$. We can assume that $\psi(B)=\psi\left(B^{\prime}\right)=\xi$. It is enough to prove that, if $b \in \mathbf{B}_{\mathcal{O}}, b^{\prime} \in \mathbf{B}_{\mathcal{O}^{\prime}}$, then $\left(b: b^{\prime}\right)^{\mathcal{Q}}=(-1)^{\operatorname{dim} \mathcal{O}+\operatorname{dim} \mathcal{O}^{\prime}}\left(b: b^{\prime}\right)$. Using 14.2 and 14.7, we have

$$
\left(b: b^{\prime}\right)^{\mathcal{Q}}=\left(b^{\odot}: b^{\prime \odot}\right)=\left((-1)^{\operatorname{dim} \mathcal{O}} b,(-1)^{\operatorname{dim} \mathcal{O}^{\prime}} b^{\prime}\right)
$$

and (a) is proved.

\subsection{We show:}

(a) For any $B, B^{\prime}$ in $\mathfrak{B}$ we have $P_{B, B^{\prime}} \in \mathbf{N}\left[v^{-2}\right], \tilde{P}_{B, B^{\prime}} \in \mathbf{N}\left[v^{-2}\right], \tilde{\Lambda}_{B, B^{\prime}} \in$ $\mathbf{Q}\left(\left(v^{2}\right)\right)$ (notation of 13.6). to

The proof is similar to that of $13.7(\mathrm{a})$. With the notation in $14.8(\mathrm{a})$ we apply $\mathrm{O}$

$$
v^{-\operatorname{dim} \mathcal{O}-\operatorname{dim} \mathcal{O}^{\prime}}\left(B: B^{\prime}\right)=\sum_{B_{1} \in \mathfrak{B}, B_{2} \in \mathfrak{B}} P_{B_{1}, B} \tilde{\Lambda}_{B_{1}, B_{2}} \tilde{P}_{B_{2}, B^{\prime}}
$$

(see 13.6(d)); using 14.8 we obtain

$$
v^{-\operatorname{dim} \mathcal{O}-\operatorname{dim} \mathcal{O}^{\prime}}\left(B: B^{\prime}\right)=\sum_{B_{1} \in \mathfrak{B}, B_{2} \in \mathfrak{B}} P_{B_{1}, B}^{\odot} \tilde{\Lambda}_{B_{1}, B_{2}}^{\odot} \tilde{P}_{B_{2}, B^{\prime}}^{\odot}
$$

When $B, B^{\prime}$ vary, this can be expressed as the decomposition of the matrix $\mathcal{M}=$ $\left(v^{c_{B}-c_{B^{\prime}}} v^{-\kappa(B)-\kappa\left(B^{\prime}\right)}\left(B: B^{\prime}\right)\right)$ (indexed by $\mathfrak{B} \times \mathfrak{B}$ ) as a product of three matrices $\mathcal{S}^{\diamond} \mathcal{T}^{\odot} \mathcal{S}^{\prime}$ where $\mathcal{S}^{\diamond}$ (resp. $\mathcal{S}^{\prime}$ ) is the matrix indexed by $\mathfrak{B} \times \mathfrak{B}$ whose $\left(B, B^{\prime}\right)$ entry is $P_{B^{\prime}, B}^{\odot}$ (resp. $\tilde{P}_{B, B^{\prime}}^{\odot}$ ) and $\mathcal{T}^{\odot}$ is the matrix indexed by $\mathfrak{B} \times \mathfrak{B}$ whose $\left(B, B^{\prime}\right)$-entry is $\tilde{\Lambda}_{B, B^{\prime}}^{\odot}$. Recall from 13.6 that we have also $\mathcal{M}=\mathcal{S} \mathcal{T} \mathcal{S}^{\prime}$ (notation of 13.6). Thus we have

$$
\mathcal{S}^{\odot} \mathcal{T}^{\odot} \mathcal{S}^{\circ}=\mathcal{S} \mathcal{T} \mathcal{S}^{\prime}
$$


Now by 13.6(c),(e), the matrix $\mathcal{T}$ (and hence the matrix $\mathcal{T}^{\odot}$ ) belongs to a subgroup of $G L_{N}(N=\sharp(\mathfrak{B}))$ of the form $G L_{N_{1}} \times \ldots G L_{N_{k}}$ where $N_{1}, \ldots, N_{k}$ are the sizes of the various subsets $\mathfrak{B}_{\mathcal{O}}$; moreover, by $13.6(\mathrm{a}),(\mathrm{b})$, the matrix $\mathcal{S}$ (hence the matrix $\mathcal{S}^{\diamond}$ ) belongs to the unipotent radical of a parabolic subgroup of $G L_{N}$ with Levi subgroup equal to the subgroup of $G L_{N}$ considered above, while the matrix $\mathcal{S}^{\prime}$ (hence the matrix $\mathcal{S}^{\circ}$ ) belongs to the unipotent radical of the opposite parabolic subgroup with the same Levi subgroup. This forces the equalities $\mathcal{S}^{\odot}=\mathcal{S}, \mathcal{T}^{\odot}=\mathcal{T}$, $\mathcal{S}^{\prime}{ }^{\odot}=\mathcal{S}^{\prime}$. Now the equality $\mathcal{S}^{\odot}=\mathcal{S}$ implies $P_{B^{\prime}, B}^{\odot}=P_{B^{\prime}, B}$ for all $B^{\prime}, B$ in $\mathfrak{B}$. Similarly, from $\mathcal{T}^{\odot}=\mathcal{T}$ we see that $\tilde{\Lambda}_{B, B^{\prime}}^{\odot}=\tilde{\Lambda}_{B, B^{\prime}}$ for all $B, B^{\prime}$ in $\mathfrak{B}$ and from $\mathcal{S}^{\prime}{ }^{\odot}=\mathcal{S}^{\prime}$ we see that $\tilde{P}_{B, B^{\prime}}^{\odot}=\tilde{P}_{B, B^{\prime}}$. This proves (a).

Theorem 14.10.

(a) Let $(\mathcal{O}, \mathcal{L}) \in \mathcal{I}\left(\mathfrak{g}_{\delta}\right)$ and let $A=\mathcal{L}^{\sharp} \in \mathcal{Q}\left(\mathfrak{g}_{\delta}\right)$. We have $\mathcal{H}^{a} A=0$ for any odd integer $a$.

(b) Let $\varpi \in \mathbf{E}^{\prime}$. We have $\mathcal{H}^{a}\left(I_{\varpi}\right)=0$ for any odd integer $a$.

(c) Let $\left(\mathfrak{p}_{*}, L, P_{0}, \mathfrak{l}, \mathfrak{l}_{*}, \mathfrak{u}_{*}\right)$ be as in $4.1(\mathrm{a})$ of $[\mathrm{LY}]$ with $\epsilon=\dot{\eta}$, and let $\left(\mathcal{O}^{\prime}, \mathcal{L}^{\prime}\right) \in$ $\mathcal{I}\left(\mathfrak{l}_{\eta}\right)$ (see 1.2). We form $A^{\prime}=\mathcal{L}^{\prime \sharp} \in \mathcal{Q}\left(\mathfrak{l}_{\eta}\right)$. We have $\mathcal{H}^{a}\left({ }^{\epsilon} \operatorname{Ind}_{\mathfrak{p}_{\eta}}^{\mathfrak{g}_{\delta}}\left(A^{\prime}\right)\right)=0$ for any odd integer $a$.

(d) In the setup of (c), ${ }^{\epsilon} \operatorname{Ind}_{\mathfrak{p}_{\eta}}^{\mathfrak{g} \delta}\left(A^{\prime}\right)$ is a direct sum of complexes of the form $\mathcal{L}^{\sharp}[s]$ for various $(\mathcal{O}, \mathcal{L}) \in \mathcal{I}\left(\mathfrak{g}_{\delta}\right)$ and various even integers $s$.

(a) follows from $14.9(\mathrm{a})$.

We prove (b). Let $\mathbf{c} \in \stackrel{\circ}{\mathbf{E}}$ be such that $\varpi \in \mathbf{c}$. In $\mathbf{V}$ we have $T_{\mathbf{c}}=\oplus_{b \in \mathbf{B}} f_{b} v^{-\kappa(b)} b$, where $f_{b} \in \mathcal{A}$. Applying ${ }^{\odot}$ and using $T_{\mathbf{c}}^{\odot}=T_{\mathbf{c}}$ and $\left(v^{-\kappa(b)} b\right)^{\odot}=v^{-\kappa(b)} b$ for $b \in \mathbf{B}$ (see 14.7) we see that

$$
\oplus_{b \in \mathbf{B}} f_{b}^{\odot} v^{-\kappa(b)} b=\oplus_{b \in \mathbf{B}} f_{b} v^{-\kappa(b)} b .
$$

Hence $f_{b}^{\odot}=f_{b}$, that is, $f_{b} \in \mathbf{Z}\left[v^{2}, v^{-2}\right]$. Thus,

(e) $I_{\varpi}$ is isomorphic to a direct sum of complexes of the form $B[-\kappa(B)][2 s]$ with $B \in \mathfrak{B}$ and $s \in \mathbf{Z}$.

Hence $\mathcal{H}^{a} I_{\varpi}$ is isomorphic to a direct sum of sheaves of the form $\mathcal{H}^{a+2 s}(B[-\kappa(B)])$ with $B \in \mathfrak{B}$. Hence the desired result follows from (a).

We prove (c). By 1.5(a) of [LY] we can find $\mathfrak{q}_{*},\left(\hat{\mathfrak{p}}_{*}, \hat{L}, \hat{P_{0}}, \hat{\mathfrak{l}}, \hat{\mathfrak{l}}_{*}, \hat{\mathfrak{u}}_{*}\right)$ as in 4.2 of [LY] with $\epsilon=\dot{\eta}$ and a cuspidal perverse sheaf $C$ in $\mathcal{Q}\left(\hat{\mathfrak{l}}_{\eta}\right)$ such that $A^{\prime}\left[s^{\prime}\right]$ is a direct summand of $\operatorname{ind}_{\mathfrak{q}_{\eta}}^{\mathfrak{l}_{\eta}}\left(C\left[-\operatorname{dim} \mathfrak{l}_{\eta}\right]\right)$ for some $s^{\prime} \in \mathbf{Z}$ hence, by $4.2\left(\right.$ a) of $[\mathrm{LY}],{ }^{\epsilon} \operatorname{Ind}_{\mathfrak{p}_{\eta}}^{\mathfrak{g}_{\delta}}\left(A^{\prime}\right)\left[s^{\prime}\right]$ is a direct summand of ${ }^{\epsilon} \operatorname{Ind}_{\hat{\mathfrak{p}}_{\eta}}^{\mathfrak{g}_{\delta}}\left(C\left[-\operatorname{dim} \mathfrak{l}_{\eta}\right]\right)$; moreover, by [L4, 21.1(c)], we have $s^{\prime}=2 s^{\prime \prime}$ for some $s^{\prime \prime} \in \mathbf{Z}$. Hence $\mathcal{H}^{a}\left({ }^{\epsilon} \operatorname{Ind}_{\mathfrak{p}_{\eta}} \mathfrak{g}_{\delta}\left(A^{\prime}\right)\right)$ is a direct summand of

$$
\mathcal{H}^{a-2 s^{\prime \prime}}\left({ }^{\epsilon} \operatorname{Ind}_{\hat{\mathfrak{p}}_{\eta}}^{\mathfrak{g} \delta}\left(C\left[-\operatorname{dim} \mathfrak{l}_{\eta}\right]\right)\right) .
$$

We can assume that $\hat{\mathfrak{p}}_{\eta}$ is an $\epsilon$-spiral with splitting $\mathfrak{m}_{*}$ (in 10.1) and that $C=\tilde{C}$ (in 10.1). Then ${ }^{\epsilon} \operatorname{Ind}_{\hat{\mathfrak{p}}_{\eta}}^{\mathfrak{g}_{\delta}}\left(C\left[-\operatorname{dim} \mathfrak{l}_{\eta}\right]\right)=I_{\varpi}$ for some $\varpi \in \mathbf{E}^{\prime}$ and $\mathcal{H}^{a}\left({ }^{\epsilon} \operatorname{Ind}_{\mathfrak{p}_{\eta}}^{\mathfrak{g}_{\delta}}\left(A^{\prime}\right)\right)$ is a direct summand of $\mathcal{H}^{a-2 s^{\prime \prime}} I_{\varpi}$. The desired result follows from (b).

We prove (d). As in the proof of $(\mathrm{c}),{ }^{\epsilon} \operatorname{Ind}_{\mathfrak{p}_{\eta}} \mathfrak{g}_{\delta}\left(A^{\prime}\right)$ is a direct summand of $I_{\varpi}\left[-2 s^{\prime \prime}\right]$ for some $\varpi \in \mathbf{E}^{\prime}$ and $s^{\prime \prime} \in \mathbf{Z}$. This, together with (e) gives the desired result. The theorem is proved. 


\section{REFERENCES}

[KL2] David Kazhdan and George Lusztig, Schubert varieties and Poincaré duality, Geometry of the Laplace operator (Proc. Sympos. Pure Math., Univ. Hawaii, Honolulu, Hawaii, 1979), Proc. Sympos. Pure Math., XXXVI, Amer. Math. Soc., Providence, R.I., 1980, pp. 185-203. MR.573434

[L4] George Lusztig, Study of perverse sheaves arising from graded Lie algebras, Adv. Math. 112 (1995), no. 2, 147-217, DOI 10.1006/aima.1995.1031. MR.1327095

[L6] George Lusztig, Cuspidal local systems and graded Hecke algebras. I, Inst. Hautes Études Sci. Publ. Math. 67 (1988), 145-202. MR.972345

[L7] George Lusztig, Character sheaves. V, Adv. in Math. 61 (1986), no. 2, 103-155, DOI 10.1016/0001-8708(86)90071-X. MR849848

[L8] G. Lusztig, Canonical bases arising from quantized enveloping algebras, J. Amer. Math. Soc. 3 (1990), no. 2, 447-498, DOI 10.2307/1990961. MR1035415

[LY] G. Lusztig and Z. Yun, Z/m-graded Lie algebras and perverse sheaves, I, Represent. Theory 21 (2017), no. 12, 277-321.

Department of Mathematics, Massachusetts Institute of Technology, Cambridge, MassachusetTs 02139

E-mail address: gyuri@math.mit.edu

Department of Mathematics, Yale University, New Haven, Connecticut 06511

E-mail address: zhiweiyun@gmail.com 